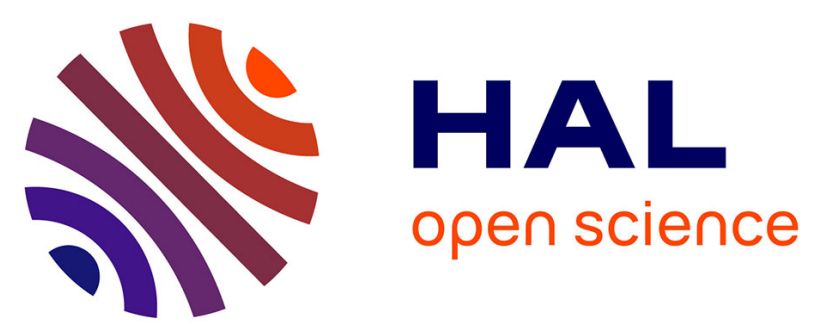

\title{
Triton Haze Analogs: The Role of Carbon Monoxide in Haze Formation
}

\author{
Sarah E. Moran, Sarah M. Hörst, Chao He, Michael J. Radke, Joshua A. \\ Sebree, Noam R. Izenberg, Véronique Vuitton, Laurène Flandinet, \\ François-Régis Orthous-Daunay, Cédric Wolters
}

\section{To cite this version:}

Sarah E. Moran, Sarah M. Hörst, Chao He, Michael J. Radke, Joshua A. Sebree, et al.. Triton Haze Analogs: The Role of Carbon Monoxide in Haze Formation. Journal of Geophysical Research. Planets, 2022, 127 (1), pp.e2021JE006984. 10.1029/2021je006984 . insu-03559249

\section{HAL Id: insu-03559249 \\ https://hal-insu.archives-ouvertes.fr/insu-03559249}

Submitted on 6 Feb 2022

HAL is a multi-disciplinary open access archive for the deposit and dissemination of scientific research documents, whether they are published or not. The documents may come from teaching and research institutions in France or abroad, or from public or private research centers.
L'archive ouverte pluridisciplinaire HAL, est destinée au dépôt et à la diffusion de documents scientifiques de niveau recherche, publiés ou non, émanant des établissements d'enseignement et de recherche français ou étrangers, des laboratoires publics ou privés.

\section{(ㄷ)(1) $\$$}

Distributed under a Creative Commons Attribution - NonCommerciall 4.0 International 


\section{RESEARCH ARTICLE} 10.1029/2021JE006984

Key Points:

- Multiple solar system bodies have complex photochemical hazes which derive from their nitrogen and carbonrich atmospheres

- We generate and measure the properties of analog hazes ("tholin") specific to Triton-like composition and temperature

- Despite other similarities, Triton tholin are much more strongly oxygenated and slightly more nitrogenated than Titan and Pluto tholin

Correspondence to:

S. E. Moran,

smoran14@jhu.edu

\section{Citation:}

Moran, S. E., Hörst, S. M., He, C., Radke, M. J., Sebree, J. A., Izenberg, N. R., et al. (2022). Triton haze analogs: The role of carbon monoxide in haze formation. Journal of Geophysical Research: Planets, 127, e2021JE006984. https://doi. org/10.1029/2021JE006984

Received 29 JUN 2021

Accepted 21 DEC 2021

Author Contributions:

Conceptualization: Sarah E. Moran, Sarah M. Hörst, Chao He, Noam R. Izenberg

Data curation: Sarah E. Moran Formal analysis: Sarah E. Moran, Sarah M. Hörst, Chao He, Michael J. Radke, Joshua A. Sebree, Véronique Vuitton Investigation: Sarah E. Moran, Sarah M. Hörst, Chao He, Michael J. Radke, Joshua A. Sebree, Noam R. Izenberg, Véronique Vuitton, Laurène Flandinet, Cédric Wolters

Methodology: Sarah E. Moran, Sarah M. Hörst, Chao He, Michael J. Radke, Joshua A. Sebree, Véronique Vuitton, Laurène Flandinet, François-Régis OrthousDaunay, Cédric Wolters

Project Administration: Sarah M. Hörst

(C) 2022 The Authors.

This is an open access article under the terms of the Creative Commons Attribution-NonCommercial License, which permits use, distribution and reproduction in any medium, provided the original work is properly cited and is not used for commercial purposes.

\section{Triton Haze Analogs: The Role of Carbon Monoxide in Haze Formation}

\author{
Sarah E. Moran ${ }^{1,2}$ (D), Sarah M. Hörst' ${ }^{1,3,4}$ iD, Chao He ${ }^{1}$ iD, Michael J. Radke ${ }^{1}$ iD, \\ Joshua A. Sebree $^{5}$, Noam R. Izenberg ${ }^{6}$ iD, Véronique Vuitton $^{7}$, Laurène Flandinet ${ }^{7}$, \\ François-Régis Orthous-Daunay ${ }^{7}$, and Cédric Wolters ${ }^{7}$ (D)
}

\begin{abstract}
${ }^{1}$ Department of Earth and Planetary Sciences, Johns Hopkins University, Baltimore, MD, USA, ${ }^{2}$ Bay Area Environmental Research Institute, NASA Ames Research Center, Moffett Field, CA, USA, ${ }^{3}$ Hopkins Extreme Materials Institute, Johns Hopkins University, Baltimore, MD, USA, ${ }^{4}$ Space Telescope Science Institute, Baltimore, MD, USA, ${ }^{5}$ Department of Chemistry and Biochemistry, University of Northern Iowa, Cedar Falls, IA, USA, ${ }^{6}$ Applied Physics Laboratory, Johns Hopkins University, Laurel, MD, USA, ${ }^{7}$ CNRS, CNES, IPAG, Université Grenoble Alpes, Grenoble, France
\end{abstract}

Abstract Triton is the largest moon of the Neptune system and possesses a thin nitrogen atmosphere with trace amounts of carbon monoxide and methane, making it of similar composition to that of the dwarf planet Pluto. Like Pluto and Saturn's moon Titan, Triton has a haze layer thought to be composed of organics formed through photochemistry. Here, we perform atmospheric chamber experiments of $0.5 \% \mathrm{CO}$ and $0.2 \% \mathrm{CH}_{4}$ in $\mathrm{N}_{2}$ at $90 \mathrm{~K}$ and 1 mbar to generate Triton haze analogs. We then characterize the physical and chemical properties of these particles. We measure their production rate, their bulk composition with combustion analysis, their molecular composition with very high resolution mass spectrometry, and their transmission and reflectance from the optical to the near-infrared with Fourier Transform Infrared (FTIR) Spectroscopy. We compare these properties to existing measurements of Triton's tenuous atmosphere and surface, as well as contextualize these results in view of all the small, hazy, nitrogen-rich worlds of our solar system. We find that carbon monoxide present at greater mixing ratios than methane in the atmosphere can lead to significantly oxygen- and nitrogenrich haze materials. These Triton haze analogs have clear observable signatures in their near-infrared spectra, which may help us differentiate the mechanisms behind haze formation processes across diverse solar system bodies.

Plain Language Summary Triton is the largest moon of the outer planet Neptune. It has a very thin atmosphere made of similar gases to the atmospheres of the dwarf planet Pluto and Saturn's moon Titan. Sunlight or high energy particles can break apart the molecules that make up these gases, which can then react to form solid particles, called hazes. We made haze particles in an atmospheric chamber under Tritonlike temperature $(90 \mathrm{~K})$ and atmospheric composition (small amounts of carbon monoxide and methane in molecular nitrogen), and then measured the chemical and physical properties of the resulting material. We compare our results to similar measurements of laboratory materials made for Pluto and Titan. Our results show larger oxygen and nitrogen contents for these Triton particles, suggesting that increasing carbon monoxide in the atmosphere changes the chemistry of hazes. Within the laboratory hazes, we see signatures of molecular bonds containing oxygen in the near-infrared, which might be useful for identifying these species with future observations of or missions to Triton.

\section{Introduction}

Triton, as Neptune's largest moon, is unique among the ice giant moons because of its thin nitrogen atmosphere and its status as a captured Kuiper Belt Object (KBO; Agnor \& Hamilton, 2006; McKinnon, 1984). Also considered a candidate ocean world, Triton is a natural comparison to two other worlds, Titan and Pluto, which also have nitrogen-rich atmospheres with trace amounts of carbon monoxide and methane, though the absolute mixing ratios differ between the three. Rich photochemistry has been observed both on Titan and Pluto from dedicated spacecraft observations by the Cassini-Huygens mission to the Saturn system and the New Horizons flyby of Pluto. Dramatic haze layers are seen in the atmospheres of both worlds from these two missions (Porco et al., 2005; Stern et al., 2015). Voyager 2 observations also suggest that Triton has a haze layer and potentially nitrogen ice clouds (Herbert \& Sandel, 1991; Rages \& Pollack, 1992; Strobel \& Summers, 1995; Yelle et al., 1995), though 
Resources: Sarah M. Hörst Software: Sarah E. Moran, Sarah M. Hörst, Véronique Vuitton, François-Régis Orthous-Daunay

Supervision: Sarah M. Hörst Validation: Sarah M. Hörst, Véronique Vuitton, Cédric Wolters

Visualization: Sarah E. Moran Writing - original draft: Sarah E. Moran

Writing - review \& editing: Sarah $\mathrm{M}$. Hörst, Chao He, Michael J. Radke, Joshua A. Sebree, Noam R. Izenberg, Véronique Vuitton, Laurène Flandinet, FrançoisRégis Orthous-Daunay, Cédric Wolters the haze properties, especially as compared to that of Titan and Pluto, remain poorly characterized due to Voyager 2 phase angle and detection limit constraints (Pollack et al., 1990).

Triton's atmosphere, due to its extremely cold surface temperature of $38 \mathrm{~K}$ (Conrath et al., 1989), is in vapor pressure equilibrium with its surface ices, making atmosphere-surface interactions active and strongly seasonally dependent (Cruikshank et al., 1993; Hansen \& Paige, 1992). Similar activity is observed for Pluto (Lewis et al., 2020), where seasonal sublimation appears to drive winds (Telfer et al., 2018). Measurements of Triton's atmospheric pressure at the surface range from $14 \pm 1 \mu$ bar in 1989 from Voyager 2 radio science (Tyler et al., 1989) to $19 \pm 1.8 \mu$ bar from stellar occultations in 1995 and 1997 (Elliot et al., 1998; Olkin et al., 1997), suggestive of seasonal sublimation and deposition. Nitrogen dominates the atmosphere, and vertical profiles of the $\mathrm{N}_{2}$ (Krasnopolsky et al., 1993) and $\mathrm{CH}_{4}$ content, which decreases with altitude (Herbert \& Sandel, 1991), have been known since Voyager 2. From the surface at $38 \mathrm{~K}$, the upper atmosphere reaches temperatures of approximately $90 \mathrm{~K}$ (Strobel \& Zhu, 2017). With the Very Large Telescope of the European Southern Observatory, Lellouch et al. (2010) was able to measure the amount of CO in the atmosphere, where only upper limits were achieved by Voyager 2. Lellouch et al. (2010) found a partial pressure of $24 \pm 3$ nbar for CO, but did not measure a surface pressure at the time of observations.

Triton's young ( $\leq 10 \mathrm{Ma}$ ) surface (Schenk \& Zahnle, 2007; Stern \& McKinnon, 2000) requires geological activity suggestive of significant internal heating, potentially provided by obliquity tides (Nimmo \& Spencer, 2015), which could maintain a subsurface liquid ocean. Plumes/geysers on Triton, observed by Voyager 2, also suggest seasonally driven winds and/or subsurface activity (Hammond et al., 2018; Hansen et al., 1990). Organic material generated by photochemistry in the atmosphere could then interact with this potential subsurface ocean, or even the plume outflow material itself, furthering prebiotic chemical reactions, as may also occur on Titan (Neish et al., 2010).

In Titan's atmosphere, the dissociation of $\mathrm{N}_{2}, \mathrm{CH}_{4}$, and other minor species that initiate haze formation is primarily driven by solar UV radiation (Vuitton et al., 2019), while galactic cosmic ray impacts can also initiate ionization reactions to a lesser degree (Gronoff et al., 2009, 2011). Magnetospheric ions from Saturn on the order of up to $1 \mathrm{keV}$ with flux $10^{-6} \mathrm{~cm}^{-2} \mathrm{~s}^{-2}$ have been detected at Titan (Hartle et al., 2006) and are a minor driver of atmospheric chemistry on the moon. However, at Triton, Neptune's magnetospheric particles interact with Triton's ionosphere with energies an order of magnitude higher (Krimigis et al., 1989; Stone et al., 1989; Thompson et al., 1989) and thus may play an increased role in atmospheric chemistry, though solar EUV photons likely still dominate (Lyons et al., 1992). The flux of solar photons naturally decreases with distance from the Sun from Titan to Triton to Pluto, from $\sim 15 \mathrm{~W} \mathrm{~m}^{-2}$ to $\sim 1.5 \mathrm{~W} \mathrm{~m}^{-2}$ to $\sim 0.9 \mathrm{~W} \mathrm{~m}^{-2}$. Determining how far organic atmospheric chemistry has proceeded on Triton as a result of haze formation processes, and how this compares to Pluto and Titan, is thus of high interest given the similarities in bulk composition yet major differences in energy distribution between the three planetary bodies.

Efforts to model the haze formation process on Pluto and Triton have shown previously that the $\mathrm{CO}$ and $\mathrm{CH}_{4}$ mixing ratios in the atmosphere act as a strong control on the overall composition of haze and photochemical gas products (Krasnopolsky, 2012; Krasnopolsky \& Cruikshank, 1995; Strobel \& Zhu, 2017). These models have noted that ethylene gas, $\mathrm{C}_{2} \mathrm{H}_{4}$, as a product of this photochemical process is likely to condense the most readily of all photochemical products considered (Krasnopolsky et al., 1992; Wong et al., 2017). Hazes of the upper atmosphere can act as thermal controls of both the atmosphere and surface (Zhang et al., 2017), which is critically dependent on the exact composition and optical properties of the material. Recent coupled photochemical and microphysical modeling suggests that gas phase, $\mathrm{C}_{2}$-based hydrocarbon adsorption onto aerosol particle surfaces (Luspay-Kuti et al., 2017) as well as ice condensation onto haze particle condensation nuclei (dominated by HCN cores) may better explain the observations of both Pluto and Triton's atmosphere than photochemical haze alone (Lavvas et al., 2021), where $\mathrm{C}_{2} \mathrm{H}_{4}$ ice would dominate the composition of these heterogeneous, coated particles on Triton (Krasnopolsky, 2020; Lavvas et al., 2021). Both spherical and fractal aggregate particles can explain Triton observations using heterogeneous haze-ice particles, though fractals are preferred (Ohno et al., 2021).

Current models are in need of additional data to better constrain these atmospheric processes, but in situ missions to the outer solar system to characterize atmospheric chemistry are both few and far between, and often only reveal far more complexity than previously assumed, as in the case of both Titan and Pluto. Moreover, because of the unique and highly complex chemistry occurring in these haze layers, theoretical models often cannot fully 
capture haze formation from initial gases to complex haze molecules due to both a lack of chemical kinetics of the relevant chemical reactions as well as computational expense (Berry et al., 2019; Vuitton et al., 2019). As such, laboratory synthesis of atmospheric hazes has been performed to generate and study analog particles to those potentially made in planetary atmospheres, including those for Titan (e.g., Imanaka et al., 2004; Khare et al., 1984; Vuitton et al., 2010), the early Earth (e.g., DeWitt et al., 2009; Hasenkopf et al., 2010; Hörst, He, Ugelow, et al., 2018; Trainer et al., 2006), and even exoplanets (e.g., Gavilan et al., 2018; Hörst, He, Lewis, et al., 2018). These experiments have revealed a wealth of information about haze formation and properties, including their effect on spectra (Brassé et al., 2015), the production of prebiotic molecules (Hörst et al., 2012; Sebree et al., 2018), and potential chemical pathways to haze formation (Gautier et al., 2014, 2016).

Previous atmospheric haze formation experiments also exist for Triton specifically (McDonald et al., 1994; Thompson et al., 1989), but these past experiments included only nitrogen and methane in their starting gas mixtures. Thanks to more recent ground-based observations (Lellouch et al., 2010), we now know that CO is in fact the second most abundant molecule in Triton's $\mathrm{N}_{2}$ atmosphere, unlike the Plutonian and Titanian atmospheres where $\mathrm{CH}_{4}$ is found in higher mixing ratios than $\mathrm{CO}$ (Krasnopolsky, 2012). Some experiments have included CO in Titan-like mixtures (Bernard et al., 2003; Coll et al., 2003; Tran et al., 2008), suggesting oxygenated molecules play a role in the gas phase chemistry. More recent laboratory experiments have shown that an initial inventory of gas phase CO also substantially affects resulting tholin particles (He et al., 2017; Hörst \& Tolbert, 2014). CO was included in recent atmospheric experiments of a Pluto-like atmosphere (Jovanović et al., 2020, 2021), with substantial impact on the tholin produced. However, this Pluto experiment, as well as the previous Triton experiments, were performed at room temperature, though temperature should also influence the chemistry occurring in the atmosphere. Moreover, the published spectra and chemical measurements from every $\mathrm{N}_{2}-\mathrm{CH}_{4}-\mathrm{CO}$ experiment to date has been performed with mixing ratios where $\mathrm{CH}_{4} \geq \mathrm{CO}$, as is more representative of Titan and Pluto rather than Triton. The only published experiments performed with $\mathrm{CO}>\mathrm{CH}_{4}$ only examined the particle density and size of the resulting solid (Hörst \& Tolbert, 2014), not its chemistry. Therefore, in this study, we perform a new set of atmospheric chamber experiments for a Triton-like atmosphere-including this second most abundant molecule of $\mathrm{CO}$ at a higher percentage than $\mathrm{CH}_{4}$ and at Triton-like temperature- to examine the chemical and physical properties of hazes that may influence Triton's atmosphere and climate, surface, and planetary evolution.

\section{Methods}

First, we generated Triton haze analog particles within the PHAZER (Planetary HAZE Research) chamber (He et al., 2017) under Triton-relevant temperature and pressure with an AC cold plasma discharge as the energy source. We subjected the resulting solid particles to combustion analysis to obtain the elemental ratios of $\mathrm{C}, \mathrm{H}$, $\mathrm{O}$, and $\mathrm{N}$ that make up the particles. Next, we performed a more in-depth analysis of the chemical composition of the solids produced in the PHAZER chamber by utilizing very high resolution Thermo Fisher Scientific LTQ-Orbitrap XL mass spectrometry. Once mass spectrometer measurements were complete, we employed custom software called idmol (Hörst, 2011) to make molecular identifications and perform data analysis from the mass spectrometry data. Finally, we obtained the transmittance and reflectance of the Triton haze analogs by measuring thin films of the particles with a Bruker Vertex 70V Fourier Transform Infrared Spectrometer.

\subsection{Triton Haze Analog Production}

With the PHAZER chamber and accompanying apparatus (shown in Figure 1) (JHU, Baltimore, MD), we produced Triton haze analog particles. We continuously flowed a gas mixture of $0.5 \% \mathrm{CO}$ (Airgas) and $0.2 \% \mathrm{CH}_{4}$ (Airgas) in $\mathrm{N}_{2}$ (Airgas) for $72 \mathrm{hr}$, as is standard PHAZER protocol (He et al., 2017) for the generation of substantial macroscopic sample. To calculate these mixing ratios, we use the partial pressure of $\mathrm{CO}$ determined by Lellouch et al. (2010), 24 nbar within a factor 3. We divide the upper limit of this partial pressure by the surface pressure determined by Voyager 2, $14 \mu$ bar, to obtain $0.5 \% \mathrm{CO}$ in our mixture. Lellouch et al. (2010) reports the surface partial pressure ratio $\mathrm{CO} / \mathrm{CH}_{4}$ to be $\sim 2.5$, resulting in our $\mathrm{CH}_{4}$ mixing ratio of $0.2 \%$. While our experimental ratio of $\mathrm{CO}$ to $\mathrm{CH}_{4}$ reflects Triton's atmosphere, their absolute values compared to $\mathrm{N}_{2}$ are higher than the atmospheric mixing ratios reported by Lellouch et al. (2010) since we include this surface pressure correction. These values are chosen to generate higher yields of tholin in a reasonable experimental timeframe. We discuss the implications of this approximation on our results in Section 4.2. 


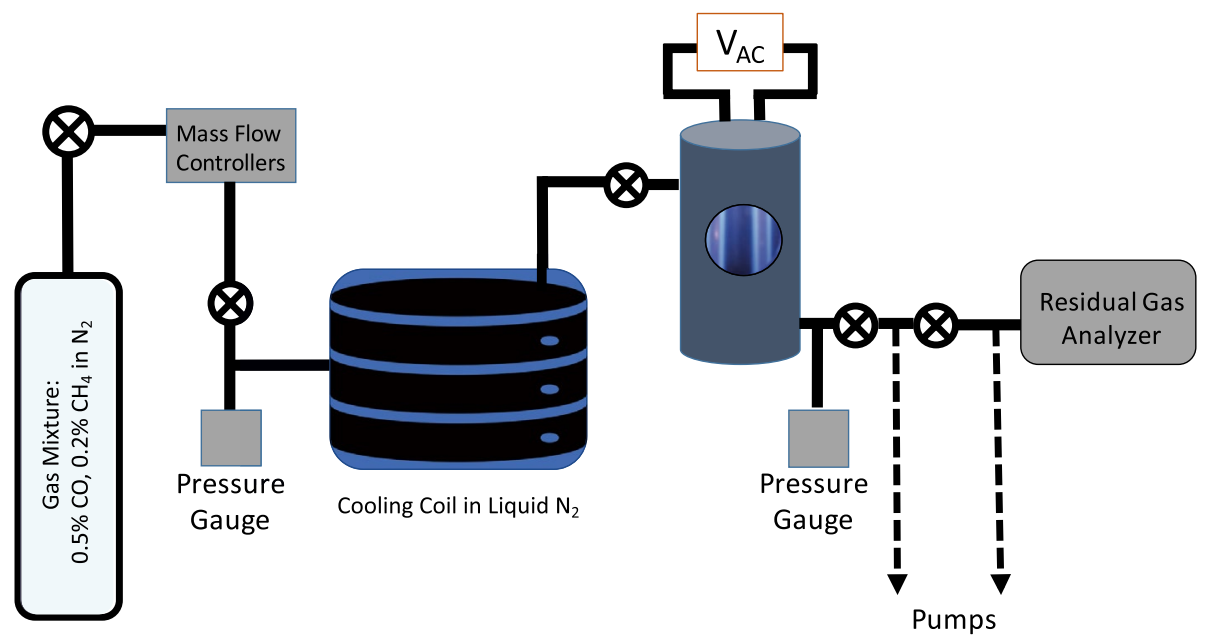

Figure 1. Schematic of the PHAZER chamber set-up at Johns Hopkins University. The $0.5 \% \mathrm{CO}, 0.2 \% \mathrm{CH}_{4}$ in $\mathrm{N}_{2}$ gas mix is flowed through the cooling coil submerged in liquid nitrogen before flowing into the reaction chamber. The mixing ratios were chosen to represent the high end of atmospheric CO from existing observations (e.g., Krasnopolsky, 2012). The mass flow controllers are set at $10 \mathrm{sccm}$ so that the pressure in the chamber is 1 mbar. The gas mixture is then exposed to the AC glow discharge, and analog haze chemistry proceeds at $90 \mathrm{~K}$.

The gas mixture first is flowed through the cooling coil immersed in a liquid $77 \mathrm{~K} \mathrm{~N}_{2}$ bath and then into the reaction chamber so that gases in the chamber are approximately $90 \pm 5 \mathrm{~K}$ (He et al., 2017), following the best fit upper atmospheric temperature determined by Strobel and Zhu (2017). This temperature corresponds to regions $300 \mathrm{~km}$ above Triton's surface where Triton's ionosphere is located (Tyler et al., 1989). On Titan, haze formation begins in the ionosphere (Lavvas et al., 2013; Liang et al., 2007), and Neptune's magnetosphere can drive similar reactions on Triton (Thompson et al., 1989). However, the main haze layer is observed much lower, from 4 to $30 \mathrm{~km}$ (Rages \& Pollack, 1992), where the atmospheric temperature is closer to $50 \mathrm{~K}$ (Strobel \& Zhu, 2017). Our laboratory set-up cannot be maintained at this low a temperature, as $90 \mathrm{~K}$ is near our experimental limit. Nevertheless, running at $90 \mathrm{~K}$ represents a much more accurate temperature condition than any previous Triton haze experiments, which have all been performed at room temperature.

An AC cold plasma glow discharge then provides an energy density of order $170 \mathrm{~W} \mathrm{~m}^{-2}$ into the system (He et al., 2019). This flux is applied over $72 \mathrm{hr}$, for a total of $4.4 \times 10^{7} \mathrm{Wm}^{-2}$ over the course of the experiment. Given that Triton receives $\sim 1.5 \mathrm{Wm}^{-2}$ in incident solar radiation, our experiment corresponds to approximately 340 days of solar irradiation at Triton. The plasma source is energetic enough to dissociate even extremely stable molecules, such as $\mathrm{N}_{2}$ and $\mathrm{CO}$ (Cable et al., 2012). This cold plasma does not directly replicate any single energetic atmospheric process, photochemical or otherwise. Instead it provides a method by which to approximate the energetics of upper atmospheres. For Triton's upper atmosphere, such energy distributions may derive from a combination of sources such as incident UV solar photons, cosmic ray bombardment, or charged particles from the magnetic field of Neptune.

The gases were flowed at 10 standard cubic centimeters per minute $(\mathrm{sccm})$ so that the pressure within the chamber was maintained at 1 mbar, which is higher than any pressure in Triton's atmosphere, which at highest is estimated to be $40 \mu$ bar (Lellouch et al., 2010). Maintaining the experimental chamber at 1 mbar pressure reduces reaction times given the mean free path within the size of the reaction chamber, allowing for completion of experimental runs within a reasonable timeframe. Furthermore, many other haze formation experiments are run at this pressure (e.g., He et al., 2017; Jovanović et al., 2020; Khare et al., 1984), which allows for comparison of the chemical and physical properties of the resulting tholins as a function only of gas species and temperature without the additional variable of pressure, which previous work has shown to affect the tholin composition and spectra. For example, Imanaka et al. (2004) found that tholin produced at lower pressures $(0.13$ mbar) incorporated more nitrogen than tholin produced at higher pressure ( $23 \mathrm{mbar}$ ), and that this led to stronger, deeper features from N-H bonds and aromatic ring structures in their spectra. 
As ionization and dissociation of the inital $\mathrm{N}_{2}, \mathrm{CO}$, and $\mathrm{CH}_{4}$ gas molecules proceeds, these ions and radicals react to make new molecules, and some combination of initial and newly created molecules generate longer and longer molecular chains. Eventually, some such molecules become macroscopic solids and deposit out in the form of orange-brown powder on the chamber walls, floor, and quartz disks placed at the bottom of the chamber. We use quartz substrates because quartz is inert and does not react with either the energy source or gas mixture during the experiment and is well characterized in our reaction chamber (He et al., 2022).

After $72 \mathrm{hr}$, the gas flow is turned off and remains under vacuum for $48 \mathrm{hr}$ to allow volatile dissipation. The chamber is then moved into a dry $\left(<0.1 \mathrm{ppm} \mathrm{H}_{2} \mathrm{O}\right)$, oxygen-free $\left(<0.1 \mathrm{ppm} \mathrm{O}_{2}\right) \mathrm{N}_{2}$ glove box (Inert Technology Inc., I-lab 2GB) where solid sample is collected and stored, insulated from ambient atmosphere and light sources. To determine the production rate of the experiment, we manually collect solid material from the chamber walls and base and weigh the total with an analytical balance (Sartorius Entris 224-1S with standard deviation of $0.1 \mathrm{mg}$ ). The production rate is then calculated by dividing the total mass by the runtime of the experiment ( $72 \mathrm{hr}$ ), with the error $(0.20 \mathrm{mg} / \mathrm{hr})$ induced from the balance and the sample residuals on the reaction chamber walls. We note also that the production rate is necessarily a lower limit as collection efficiency is always less than 1 . Samples were stored in the glovebox for $\sim 6$ months prior to mass spectrometry measurements, $\sim 21$ months prior to spectroscopy measurements, and $\sim 30$ months prior to combustion measurements. Agreement between combustion analysis results and Orbitrap mass spectrometry results, discussed in Section 3.2, confirms that external chemical alteration does not take place in storage in the glovebox.

\subsection{Combustion Analysis}

We employed elemental combustion analysis with a Thermo Scientific Flash 2000 Elemental Analyzer (Department of Chemistry and Biochemistry, University of Northern Iowa, IA, USA) of the Triton haze analogs produced in the PHAZER chamber. We placed $1 \mathrm{mg}$ of particles in the analyzer to directly measure percentages of $\mathrm{C}, \mathrm{H}$, and $\mathrm{N}$. We then perform mass subtraction to calculate the percentage of $\mathrm{O}$.

\subsection{Fourier Transform Infrared Spectroscopy}

Using a Bruker Vertex 70V Fourier Transform Infrared Spectrometer (JHU, Baltimore, MD), we measured the transmission and reflectance of thin films of Triton haze analog deposited on quartz substrates from 0.4 to 5 microns. From 0.4 to 1.1 microns, we use a Si-diode detector while from 0.83 microns to 5 microns, we employ a DLaTGS detector. Overlap between detectors allows for calibration between the wavelength ranges. The spectrometer is fitted with a quartz beamsplitter and uses a near-IR source of a tungsten-halogen lamp. A silicon carbide globar provides the mid-IR source. When performing measurements, we vent the optical bench to $\leq 1 \mathrm{hPa}$. All measurements were performed at room temperature, monitored and held stable at $294 \mathrm{~K}$. We first take transmission and reflectance measurements of a blank quartz disc to provide a baseline correction before measuring the Triton sample deposited on the quartz substrate. We use a source aperture size of $2 \mathrm{~mm}$ and average over 250 scans for each measurement. With the instrument settings as configured, our measurements have a resolution of $2 \mathrm{~cm}^{-1}$. Interference fringes are observed in the optical to near-IR range ( 0.45 micron to 2 micron), and we perform a correction for this fringing following the moving average method of Neri et al. (1987), as in He et al. (2022). This correction is given as

$$
F\left(x_{n}\right)=\frac{1}{4}\left(2 G\left(x_{n}\right)+G\left(x_{n+m}\right)+G\left(x_{n-m}\right)\right)
$$

where $\mathrm{F}\left(\mathrm{x}_{n}\right)$ is the fringe-removed spectrum, $\mathrm{G}\left(\mathrm{x}_{n}\right)$ is the original spectrum, and $2 m$ is the number of integer points in $d$, the average fringe spacing, which is $2,260 \mathrm{~cm}^{-1}$. With spectral resolution of $2 \mathrm{~cm}^{-1}$, we find $m$ to be 565 for our transmission and reflectance spectra. For completeness, we provide the uncorrected spectrum with fringes visible in Appendix C.

Once fringe removal is complete, we then compare the spectral features observed in the Triton tholin to both previous results of relevant planetary haze analogs as well as to general spectral databases in order to identify chemical bonds and functional groups. 


\subsection{Orbitrap Mass Spectrometry}

To investigate the detailed chemical composition of the Triton haze analog sample, we employed very high resolution mass spectrometry with a Thermo Fisher Scientific LTQ-Orbitrap XL mass spectrometer with an Ion Max electrospray ionization source (ESI; IPAG, Grenoble, FR), which has resolving power $(\mathrm{m} / \Delta \mathrm{m})$ of at least $10^{5}$ up to $m / z 400$ and an exact mass accuracy of $\pm 2 \mathrm{ppm}$. Prior to sample measurement, we performed mass calibration between $\mathrm{m} / \mathrm{z} 200$ and 2000 with Thermo Fisher Scientific Calmix (caffeine, MRFA peptide, and Ultramark 1621 for positive mode and sodium dodecyl sulfate, sodium taurocholate, and Ultramark 1621 in negative mode) solution. We also measured a blank solution from $m / z, 150-1000$ of pure $\mathrm{CH}_{3} \mathrm{OH}$ with the Orbitrap to account for and remove any potential contamination in sample signal from the mass calibration solution, ambient conditions, or the sample vial. To prepare the sample, we dissolved the Triton haze analog in $\mathrm{CH}_{3} \mathrm{OH}$ (methanol) at a concentration of $1 \mathrm{mg} / \mathrm{mL}$. We then subjected the dissolved sample to sonification for $1 \mathrm{hr}$, followed by centrifugation at $10,000 \mathrm{rpm}$ for $10 \mathrm{~min}$. The soluble fraction of the Triton sample was then diluted again in $\mathrm{CH}_{3} \mathrm{OH}$ at $1 \mathrm{mg} / \mathrm{mL}$. We injected the diluted soluble fraction into the Orbitrap with electrospray ionization (ESI) and obtained overlapping mass-to-charge $(\mathrm{m} / \mathrm{z}$ ) bin measurements of $\mathrm{m} / \mathrm{z}, 50-300, \mathrm{~m} / \mathrm{z}, 150-450$, and $\mathrm{m} / \mathrm{z}, 400-1000$. The tube lens voltage was set to 50,70, and $90 \mathrm{~V}$ respectively, with source voltages of $3.5 \mathrm{kV}$ (positive) and $3.8 \mathrm{kV}$ (negative). For each mass-to-charge bin, our measurements averaged together four scans of 128 microscans, with the injection of sample set at a flow rate of $3 \mu \mathrm{L} \mathrm{min}{ }^{-1}$, to maximize signal and reduce noise (Wolters et al., 2020). Initial measurements were taken in positive ion mode. The instrument polarization was then switched to negative ion mode and allowed to re-equilibrate for $90 \mathrm{~min}$. With the instrument in standby, the capillaries were flushed with $10 \mu \mathrm{L}$ of $\mathrm{CH}_{3} \mathrm{OH}$ and then the direct injection polyetheretherketone (PEEK) capillary replaced. The instrument was then mass calibrated again and another blank solution was taken in negative polarity before the Triton sample was measured in negative ion mode with the same instrument settings as above.

\subsection{Data Analysis of Orbitrap MS With Idmol}

After data acquisition and preliminary inspection with Thermo Fisher Scientific XCalibur software, we use custom IDL/Fortran software called idmol (Hörst, 2011) to extract and analyze the mass spectral data. First, we compare the mass spectra of blank solution to that of the Triton analog and remove contaminant peaks with intensities greater than $2 \times 10^{5}$ in the Triton spectra. More recent work suggests that this method of blank subtraction can remove useful signal due to the behavior of the automatic gain control (AGC) of the Orbitrap (see Wolters et al., forthcoming), but in this case we do not remove many peaks and therefore expect minimal impact on our results (see Appendix B, Figure B1). Next, we use idmol to assign molecular peaks, where the program calculates all potential molecular combinations and then uses a series of decision trees based on user inputs regarding minimum N/C ratio, maximum number of oxygens, mass tolerance, and the nitrogen rule to make assignments of low-mass mass peaks. Above $m / z, 300$, where non-unique mass peaks are no longer within the instrument's resolving power (Gautier et al., 2014), the program uses lower mass peak assignments to choose best fit assignments by eliminating potential molecules which substantially deviate from the average number of carbons, nitrogens, and double bond equivalent for the previous 20 assignments (Hörst, 2011).

As in previous analyses (Moran et al., 2020; Vuitton et al., 2021), we calculate the elemental composition of the tholin sample from Orbitrap measurements using idmol peak assignments weighted by the spectral intensity. We perform a correction to the intensity weighted elemental composition by multiplying the lowest $10 \%$ intensities by 10 to account for differing ionization yields of oxygen molecules, as was shown by Hörst (2011) to mitigate differences between Orbitrap and combustion derived elemental compositions. We also note that additional factors can introduce uncertainty into elemental ratios derived from Orbitrap results, such as the solubility of sample or the efficiency of ion transmission, in addition to the inherent error accounted for with our formula assignments. We also calculate the non-intensity weighted elemental composition, as ionization efficiency may prevent a direct proportionality to molecular concentration and impact the derived elemental ratios. For further discussion regarding ionization efficiencies of various chemical species, including as a function of positive and negative polarities, see Vuitton et al. (2021). 

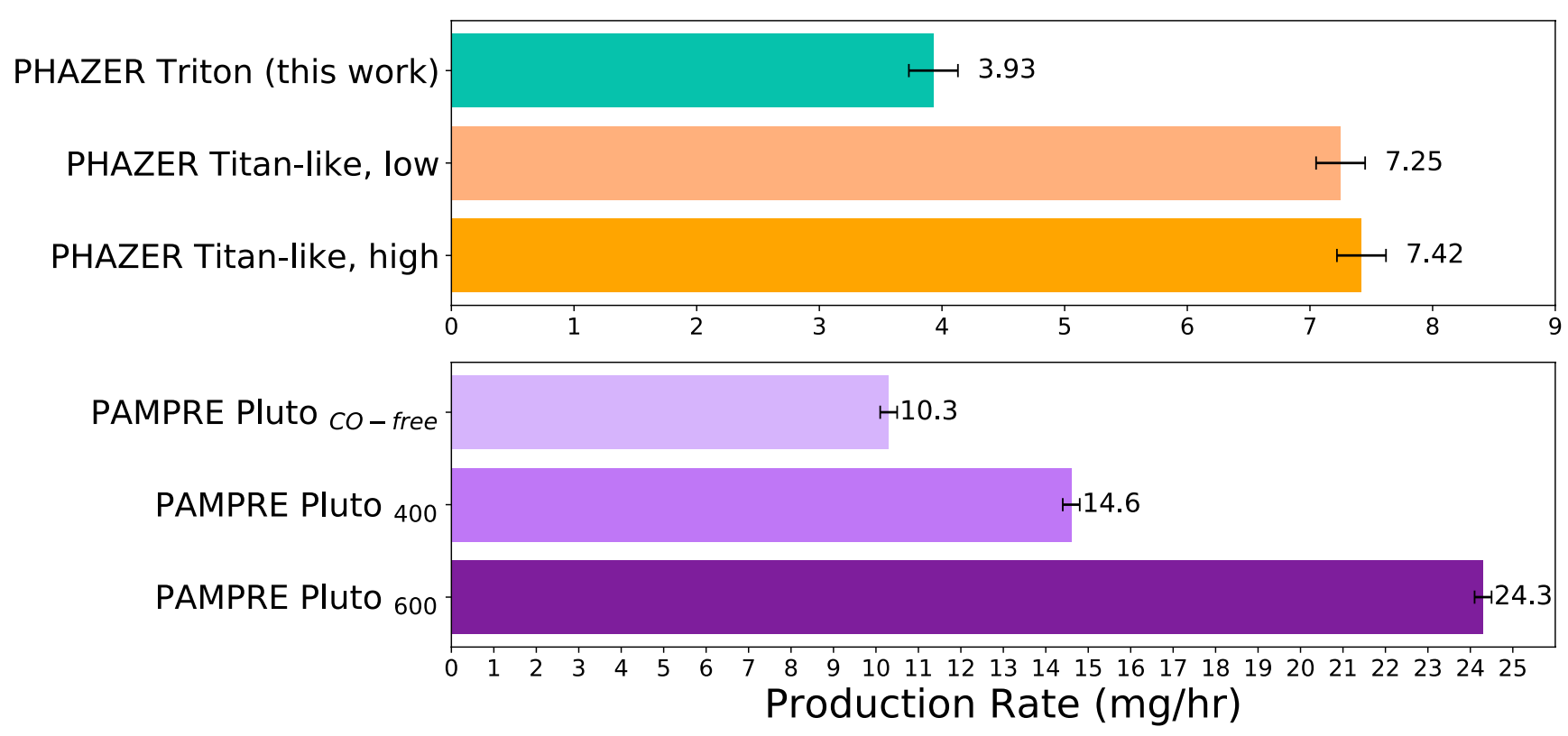

Figure 2. Top: PHAZER production rates (in $\mathrm{mg} / \mathrm{hr})$ of the amount of solid produced from the Triton gas mixture $\left(\mathrm{CO}=0.5 \%\right.$; $\mathrm{CH}_{4}=0.2 \%$; top bar in turquoise) compared to our Titan-like gas mixture $\left(\mathrm{CH}_{4}=5 \%\right.$; $\mathrm{CO}$ in varying mixing ratios from $0 \%$ to 5\%; He et al., 2017, middle and lower orange bars). Bottom: PAMPRE production rates from the room temperature Pluto-like gas mixtures $\left(\mathrm{P}_{\mathrm{CO}-\text { free }}: \mathrm{CO}=0 \%, \mathrm{CH}_{4}=1 \%\right.$, top pale purple bar; $\mathrm{P}_{400}: \mathrm{CO}=500$ ppm; $\mathrm{CH}_{4}=1 \%$, middle purple bar; and $\mathrm{P}_{600}: \mathrm{CO}=500 \mathrm{ppm}, \mathrm{CH}_{4}=5 \%$, lower dark purple bar) of Jovanović et al. (2020). Note that due to differing experimental set-ups, the absolute production rates between the PHAZER and PAMPRE apparatus cannot be directly compared and have different $x$-axis limits. While CO alters the haze chemistry, higher methane mixing ratios generate larger amounts of haze material in both experimental set-ups.

\section{Results}

In this section, we present the results of the production rate, our elemental, mass spectrometry, and Fourier Transform Infrared (FTIR) spectroscopy analysis. Broadly, CO from the initial gas mixture profoundly influences the resulting composition and slightly alters the spectra of the solid particles. First, we discuss production rate of haze as a function of $\mathrm{CO}$ and $\mathrm{CH}_{4}$ mixing ratios. Then we focus on the bulk composition as determined by both combustion and high resolution mass spectrometry (HRMS). Next we delve deeper into the composition and specific molecular identifications enabled by HRMS, and finally we examine the Triton tholin FTIR spectra for signs of the novel chemistry that may be observable by remote sensing.

\subsection{Production Rate of Triton Tholin Particles}

In Figure 2, we show the production rates of our Triton tholin experiment compared to that of our standard Titan gas mixture (5\% $\mathrm{CH}_{4}$ in $\mathrm{N}_{2}$ ) with varying $\mathrm{CO}$ percentages (He et al., 2017). While the production rates measured by $\mathrm{He}$ et al. (2017) found that varying $\mathrm{CO}$ from $0 \%$ to $5 \%$ with $5 \% \mathrm{CH}_{4}$ did not impact the production rate within the limits of experimental error $(0.20 \mathrm{mg} / \mathrm{hr})$, our Triton experiment made markedly less haze, at only $3.93 \pm 0.2 \mathrm{mg} / \mathrm{hr}$ compared to the Titan-like production rates of $7.25-7.42 \mathrm{mg} / \mathrm{hr}$. We also show the production rates (L. Jovanović, personal communication) of tholin experiments for Pluto gas composition from the PAMPRE experimental set-up (Alcouffe et al., 2009; Szopa et al., 2006) at LATMOS, which used 500 ppm CO in $\mathrm{N}_{2}$ with either $1 \% \mathrm{CH}_{4}$ to simulate Pluto's atmosphere at altitude of $400 \mathrm{~km}$ or $5 \% \mathrm{CH}_{4}$ for an altitude of $600 \mathrm{~km}$, as well as a CO-free experiment with $99 \% \mathrm{~N}_{2}$ and $1 \% \mathrm{CH}_{4}$ (Jovanović et al., 2020), all at room temperature. A description of the experimental conditions for both sets of experiments can also be found in Section 3.3.2 in Table 3. Like our Triton and Titan results, the Pluto-like results show a much higher production rate when the methane mixing ratio is higher (14.6 mg/hr with $1 \% \mathrm{CH}_{4}$ compared to $24.3 \mathrm{mg} / \mathrm{hr}$ with $5 \% \mathrm{CH}_{4}$.) While the differing experimental setups prevent a direct comparison between the absolute production rates seen in PHAZER and PAMPRE, a trend in the relative yields (i.e., normalizing the PAMPRE values to the PHAZER values as in Figure 2) is suggested. The much lower starting mixing ratio of methane in the Triton mixture $(0.2 \%)$ and low-altitude Pluto composition mixture (1\%) results in much less haze compared to that of our Titan experiments or high-altitude Pluto-like 
experiments with 5\% methane. Even more striking is the effect of removing CO from the system entirely, as seen in the CO-free, $1 \% \mathrm{CH}_{4}$ in $\mathrm{N}_{2}$ experiment of Jovanović et al. (2020). Despite the same amount of methane as in their Pluto ${ }_{400}$ condition, their CO-free condition made just $10.3 \mathrm{mg} / \mathrm{hr}$ of solid. In fact, the relative yield of their $\mathrm{CO}$-free experiment is lower than our Triton experiment which has an order of magnitude less starting $\mathrm{CH}_{4}$, but an order of magnitude more starting $\mathrm{CO}$.

Other investigations of $\mathrm{N}_{2}-\mathrm{CH}_{4}$ mixtures have shown that aerosol mass loading is always higher with $2 \% \mathrm{CH}_{4}$ than with $0.1 \% \mathrm{CH}_{4}$ using a spark discharge energy source, though the opposite is observed when the energy source is a UV lamp (Hörst \& Tolbert, 2013). Given the Triton and Titan PHAZER experiments, our glow discharge is thus likely more analogous to the spark discharge, where the larger methane gas mixing ratio allows for the production of more solid material. In a later experiment with the same set-up, the total abundance of gas phase products also increased with higher methane mixing ratios in the initial gas mixture (Hörst, Yoon, et al., 2018). PHAZER exoplanet atmospheric studies have also shown that higher initial methane mixing ratios can increase solid material production (Hörst, He, Lewis, et al., 2018), though the third highest observed production rate of those experimental conditions did not include an initial gas phase inventory of methane at all. Additional Pluto-like composition experiments (of $500 \mathrm{ppm} \mathrm{CO}$ in $\mathrm{N}_{2}$ ) observed significantly thicker thin films were produced under $5 \% \mathrm{CH}_{4}$ conditions than with $0.5 \%$ or $1 \% \mathrm{CH}_{4}$ (Jovanović et al., 2021). We also note that all these experiments have been performed at highly varying temperature conditions (90-800 K), adding a further layer of complication in extrapolating trends. Prior work has shown that Titan's methane mixing ratio appears to most efficiently convert gas precursors to tholin solids due to competing solid growth mechanisms when $\mathrm{CH}_{4}$ increases beyond this percentage (Sciamma-O'Brien et al., 2010; Trainer et al., 2006). The exact percentage of methane represented in the lab depends on the optical depth of the set-up in question (Trainer et al., 2006).

While we clearly demonstrate in later sections that the haze chemistry changes under the influence of very small amounts of $\mathrm{CO}$, the literature suggests $\mathrm{CH}_{4}$ may play a larger part in the ultimate aerosol mass loading. Current trends in the literature when combining $\mathrm{CO}$ and $\mathrm{CH}_{4}$ remain tentative or contradictory and could benefit from further study (see e.g., Hörst \& Tolbert, 2014). Early Earth studies have also examined the effect of combining methane with carbon dioxide, finding that a certain amount of $\mathrm{CO}_{2}$ with $\mathrm{CH}_{4}$ actually increases particle production up to a $\mathrm{CH}_{4} / \mathrm{CO}_{2}$ ratio of 1 before decreasing (Trainer et al., 2006), though particles are still produced even when the $\mathrm{CO}_{2}$ ratio is much higher (DeWitt et al., 2009). Therefore, while we identify the methane mixing ratio as the major driver behind increased production, it remains unclear if any carbon source in high enough quantities could serve to generate substantial haze (see, e.g., He et al., 2018, 2020; Hörst, He, Lewis, et al., 2018). This is underscored with the marked decrease in production rate when $\mathrm{CO}$ is removed entirely with a low methane mixing ratio as in Jovanović et al. (2020)'s CO-free Pluto experiment. Clearly the balance of the two carbon sources is critical to the ultimate haze mass loading.

\subsection{Composition of Triton Tholin Particles}

\subsubsection{Bulk Composition and Differences in Ion Polarities}

We observe first that despite $\mathrm{CO}$ being present at only a $0.5 \%$ mixing ratio in the initial gas mixture, the bulk solid is approximately $10 \%$ oxygen. From the combustion analysis, we determine that $\mathrm{H}, \mathrm{O}, \mathrm{C}$, and $\mathrm{N}$ are present at $5.29 \pm 0.06 \%, 10.3 \pm 0.3 \%, 43.7 \pm 0.4 \%$, and $40.7 \pm 0.4 \%$ respectively. The bulk composition as calculated from the combined positive and negative ions of the soluble fraction of the tholin from Orbitrap is $5.4 \pm 0.7 \% \mathrm{H}$, $9.8 \pm 4.3 \% \mathrm{O}, 47.4 \pm 1.7 \% \mathrm{C}$, and $37.4 \pm 2.8 \% \mathrm{~N}$. The bulk composition from the Orbitrap agrees to within $2 \%$ or better with the combustion analysis. This suggests that the soluble fraction of the Triton tholin, as measured from Orbitrap HRMS, is fairly representative of the global sample from an elemental perspective. Previous work has shown that the soluble and insoluble fractions of tholin can be quite different chemically (Maillard et al., 2018). The similarity of our elemental analyses as calculated from Orbitrap and combustion demonstrates that this effect is likely minor for our Triton tholin, though it is possible that even with the same elemental composition, the chemical composition and structure can still be quite different between the soluble and insoluble fractions.

As seen in previous work (Hörst, 2011), oxygen is primarily detected in the negatively ionized products from the Orbitrap, which is consistent with the identification of carboxylic acid groups (discussed further in the following subsections) which are efficient proton donors (Vuitton et al., 2021). The bulk values obtained for negative, positive, and combined ions from Orbitrap, as well as from combustion analysis, are shown in Figure 3. 


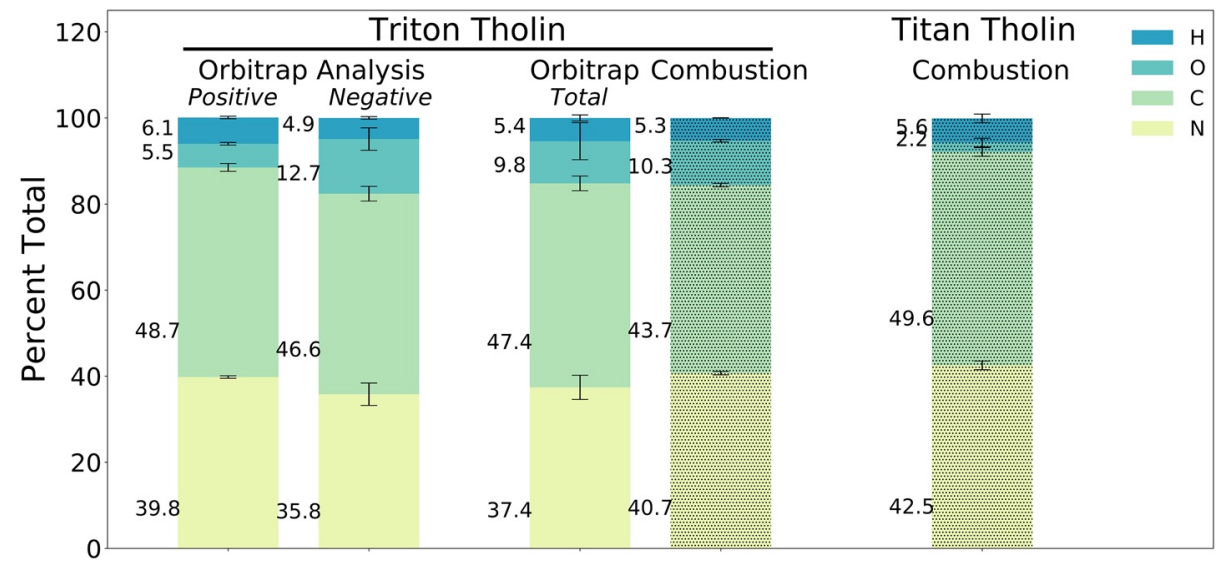

Figure 3. Average elemental composition of Triton tholin from positive, negative, and combined ions, as determined by Orbitrap MS analysis (left three bars), combustion analysis (fourth bar), and comparison to standard PHAZER Titan values from combustion analysis (right). Triton tholin more strongly incorporates oxygen, apparently through carbon depletion. Hatching on the right two bars indicates these results come from combustion analysis.

Titan tholin produced without $\mathrm{CO}$ from the same experimental set-up contains $\sim 2 \%$ oxygen (likely due to minor water adsorption during measurements) compared to the Triton tholin with $\sim 10 \%$ oxygen (Figure 3 ). Previous PHAZER CO experiments (He et al., 2017) have included up to $5 \% \mathrm{CO}$ in $\mathrm{N}_{2}-\mathrm{CH}_{4}$ mixtures, yet never observe more than $8 \%$ oxygen in the solid and indeed measure only $5 \%$ oxygen in mixtures with starting $\mathrm{CO}$ at $0.5 \%$ as in this work. Importantly, these prior experiments were conducted with constant $\mathrm{CH}_{4}$ gas mixing ratios of 5\% and did not include a case where $\mathrm{CO}>\mathrm{CH}_{4}$. Thus our Triton haze analogs demonstrate how much more strongly oxygen can be incorporated into the solid when $\mathrm{CO}>\mathrm{CH}_{4}$ in the starting gas mixture. Similar results from $\mathrm{CO}_{2}-$ $\mathrm{CH}_{4}$ experiments also show a marked chemical transition when $\mathrm{CH}_{4} / \mathrm{CO}_{2}$ reaches unity (Trainer et al., 2006). Hydrogen contents remain at approximately $5 \%$ across varying $\mathrm{CO} / \mathrm{CH}_{4}$ starting ratios (He et al., 2017), and our Triton results here support this trend, which is also seen when $\mathrm{CO}_{2}$ and $\mathrm{H}_{2}$ ratios are varied with methane (DeWitt et al., 2009). Our elemental analysis results in a bulk N/C ratio for the Triton tholin of 0.93, which is higher than that of "standard" PHAZER Titan tholin at N/C $=0.85$. This result is surprising, considering that past work with $\mathrm{N}_{2}-\mathrm{CH}_{4}-\mathrm{CO}$ mixtures has postulated that the competition between $\mathrm{N}_{2}$ and $\mathrm{CO}$ chemistry results in oxygen incorporation primarily at the expense of nitrogen incorporation (He et al., 2017; Jovanović et al., 2020). Here, instead, carbon appears depleted with respect to the Titan tholin structure. While this could simply result from $\mathrm{CO}>\mathrm{CH}_{4}$ in the gas mixture, this result is more in line with previous work that explored the addition of $\mathrm{CO}_{2}$ and $\mathrm{O}_{2}$ in $\mathrm{N}_{2}-\mathrm{CH}_{4}$ atmospheres (Hörst, He, Ugelow, et al., 2018), which found increasingly oxidized gas mixtures increased nitrogen fixation in the solid. A previous Triton experiment also showed stronger nitrogen incorporation compared to Titan tholin (McDonald et al., 1994), though that experiment contained no CO and simply reflects the lower methane mixing ratio of the starting gas mixture. For PHAZER Titan tholin, the C/O ratio of the bulk solid is 22.5 but the Triton tholin's bulk C/O is only 4.2. Even the previous PHAZER CO experimental series with the highest starting $\mathrm{CO} / \mathrm{CH}_{4}$ gas ratio (1:1) resulted in a $\mathrm{C} / \mathrm{O}$ ratio for the tholin of $\sim 6$, considerably higher than we observe for the Triton tholin. In our Triton case, it remains unclear therefore whether the higher nitrogen and oxygen contents we see derive from the lower total carbon abundance in the starting gas mixture or the presence of $\mathrm{CO}$ in particular.

\subsubsection{Molecules in the Triton Sample From HRMS}

Following our characterization of the bulk Triton haze analog sample and its bulk soluble fraction, we examined the mass spectral data for specific molecular formulas of interest. The positive and negative mass spectra are shown in Figure 4. We detect thousands of individual peaks across both ionization polarities, indicating the high complexity of the Triton tholin as is the case with its Titan, Pluto, and exoplanet counterparts (e.g., Gautier et al., 2016; He et al., 2017; Jovanović et al., 2020; Moran et al., 2020). Distinct periodicity is observed in the peak groupings, with a repeating pattern between 13 and 14 amu across both the positive and negative ions. Positive ions have peak group periods that average to $13.57 \mathrm{amu}$; negative ions cluster in peak groupings with average spacing of $13.66 \mathrm{amu}$. These values are quite close to the $13.5 \mathrm{amu}$ observed by Hörst (2011) and consistent with 


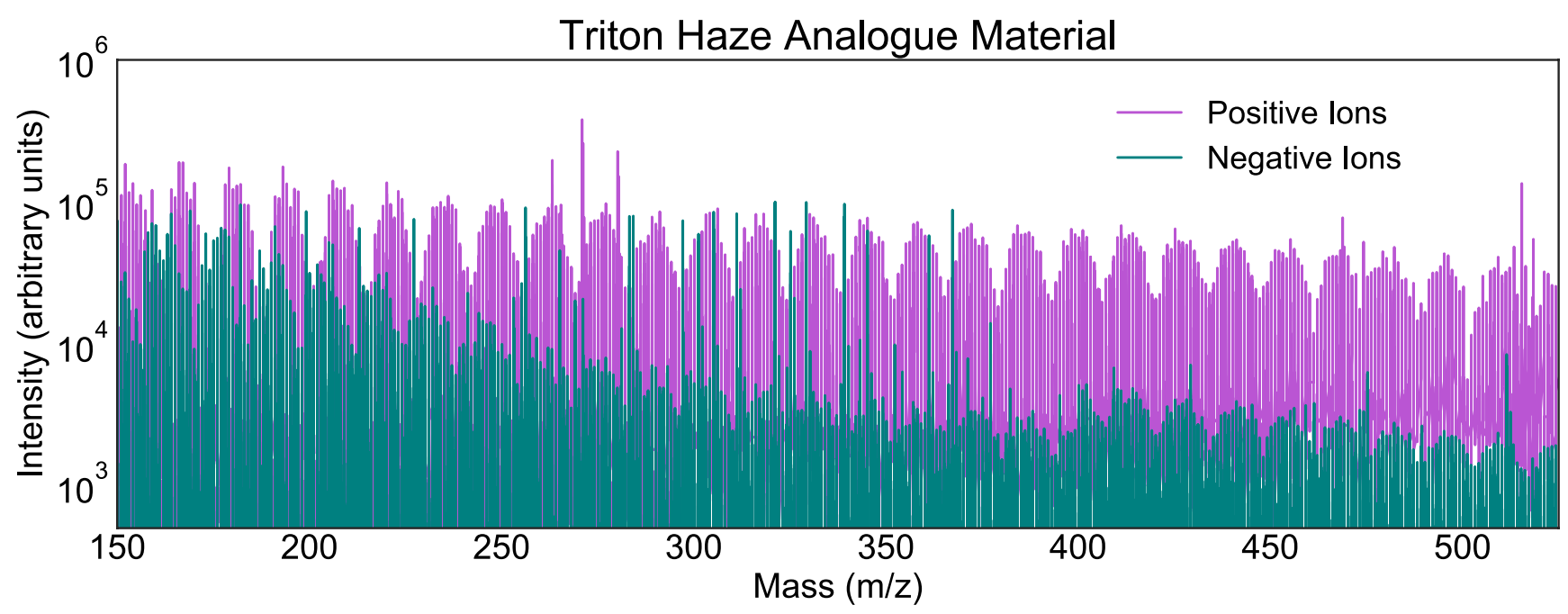

Figure 4. Positive (magenta) and negative (teal) ion mode mass spectra of the Triton haze analog particles from $\mathrm{m} / \mathrm{z} 150$ to 525 . Due to differing ionization efficiencies, the negative ion mode intensities are systematically lower. Peak groupings of $\sim 13.5$ amu repeat across the observed mass range.

the frequently observed 12-16 amu spacing (averaging to $14 \mathrm{amu}$ ) in Titan and Pluto tholin produced in various set-ups (Cable et al., 2012; Gautier et al., 2014, 2017; Imanaka et al., 2004; Jovanović et al., 2020). These peak spacings display quite regular but not exact amu periodicity. Therefore, these groupings likely indicate repeating chemical units of co-added and substituted monomers (such as $\mathrm{HCN}, \mathrm{CH}_{2}$, etc.; Gautier et al., 2017).

We report in Table 1 a small subset of the thousands of molecular formulas of species identified from Orbitrap MS with intensities $\geq 10^{3}$. These molecular formulas are consistent with those of various prebiotic molecules, though we stress that without structural information (which is not possible with the measurements conducted in this work), we cannot confirm that the formula we identify is in fact the isomer of the prebiotic species indicated in Table 1 . We detect the formulas for all biologically relevant nucleotide bases (adenine, $\mathrm{C}_{5} \mathrm{H}_{5} \mathrm{~N}_{5}$; cytosine, $\mathrm{C}_{4} \mathrm{H}_{5} \mathrm{~N}_{3} \mathrm{O}$; guanine, $\mathrm{C}_{5} \mathrm{H}_{5} \mathrm{~N}_{5} \mathrm{O}$; thymine, $\mathrm{C}_{5} \mathrm{H}_{6} \mathrm{~N}_{2} \mathrm{O}_{2}$; and uracil, $\mathrm{C}_{4} \mathrm{H}_{4} \mathrm{~N}_{2} \mathrm{O}_{2}$ ) and one non-biologic nucleotide base (xanthine, $\mathrm{C}_{5} \mathrm{H}_{4} \mathrm{~N}_{4} \mathrm{O}_{2}$ ). We note that while we include the formulas for uracil and thymine because they represent the remaining nucleotide bases, they are present only at $10^{2}$ intensities. Each of these species has been previously identified and confirmed through alternate measurement techniques in prior tholin produced from $\mathrm{N}_{2}-\mathrm{CH}_{4}-\mathrm{CO}$ mixtures (Hörst et al., 2012; Sebree et al., 2018), raising the likelihood that the formulas we identify in the Triton tholin could be the prebiotic species in question. We also identify numerous formulas consistent with isomers of amino acids and their derivatives. Nearly all of these formulas are also present as at least one ion in HRMS data of tholin produced under CO-free Titan conditions with the PHAZER set-up, as indicated in the far right column of Table 1. Because we identify most of these oxygenated molecules in the CO-free Titan sample as well as in our Triton sample, we cannot assume they are formed during sample synthesis, but must consider that they could be the result of contamination. Despite this possibility, many of these formulas have been unambiguously identified from CO-containing Titan experiments (e.g., Hörst et al., 2012) by using ${ }^{18} \mathrm{O}$ labeled CO, which we could employ in future studies to eliminate contamination concerns.

In contrast to these ambiguous detections, and most notably, we detect here the formula $\mathrm{C}_{3} \mathrm{H}_{6} \mathrm{O}_{3}$ with a potential isomer of glyceraldehyde, the simplest possible monosaccharide. This species' formula is unique to the Triton tholin among all $\mathrm{N}_{2}-\mathrm{CH}_{4}-\mathrm{CO}$ experiments to which we compare our results. Only prior tholin work considering significantly more oxidizing atmospheres has previously observed sugar formulas (Moran et al., 2020). Again, this result underscores the impact of a starting $\mathrm{CO} / \mathrm{CH}_{4}$ mixing ratio over unity. If confirmed, the existing co-presence of nucleotide bases, amino groups, and sugar molecules in the complex solids that make up this haze should significantly advance Triton as a target of astrobiological interest. For each identified formula in Table 1, we confirm that it is not present in the Orbitrap data of the blank solvent above the noise level (intensities $\geq 10^{1}$ ).

In Figure 5, we present van Krevelen diagrams (van Krevelen, 1950) using H/C versus N/C ratios of the identified molecules, which are a modification frequently used in the planetary literature to study complex organic materials like tholin (e.g., Gautier et al., 2014; Hörst, 2011; Jovanović et al., 2020). In Figure 6, we show H/C 
Table 1

Molecular Formulas Identified by Orbitrap High Resolution Mass Spectrometry

\begin{tabular}{|c|c|c|c|c|c|}
\hline$m / z \pm$ & $\Delta \mathrm{ppm}$ & Ion formula & Intensity & Potential molecule & In PHAZER Titan sample? \\
\hline 90.0312 & 0.18 & $\mathrm{C}_{3} \mathrm{H}_{6} \mathrm{O}_{3}^{-}$ & $9.4 \times 10^{3}$ & Glyceraldehyde & No \\
\hline 111.043 & -0.45 & $\mathrm{C}_{4} \mathrm{H}_{5} \mathrm{~N}_{3} \mathrm{O}^{+}$ & $1.8 \times 10^{4}$ & Cytosine & Yes \\
\hline 112.027 & 0.18 & $\mathrm{C}_{4} \mathrm{H}_{4} \mathrm{~N}_{2} \mathrm{O}_{2}^{-}$ & $6.9 \times 10^{2}$ & Uracil & Yes \\
\hline 126.043 & 0.21 & $\mathrm{C}_{5} \mathrm{H}_{6} \mathrm{~N}_{2} \mathrm{O}_{2}^{-}$ & $8.0 \times 10^{2}$ & Thymine & Yes \\
\hline \multirow[t]{2}{*}{135.054} & -0.97 & $\mathrm{C}_{5} \mathrm{H}_{5} \mathrm{~N}_{5}^{+}$ & $1.6 \times 10^{4}$ & Adenine & No \\
\hline & 0.43 & $\mathrm{C}_{5} \mathrm{H}_{5} \mathrm{~N}_{5}^{-}$ & $9.3 \times 10^{3}$ & & Yes \\
\hline \multirow[t]{2}{*}{151.049} & -1.56 & $\mathrm{C}_{5} \mathrm{H}_{5} \mathrm{~N}_{5} \mathrm{O}^{+}$ & $9.0 \times 10^{3}$ & Guanine & No \\
\hline & -0.10 & $\mathrm{C}_{5} \mathrm{H}_{5} \mathrm{~N}_{5} \mathrm{O}^{-}$ & $7.4 \times 10^{4}$ & & Yes \\
\hline 151.063 & 0.90 & $\mathrm{C}_{8} \mathrm{H}_{9} \mathrm{NO}_{2}^{-}$ & $2.5 \times 10^{3}$ & 2-phenylglycine & No \\
\hline 152.033 & -2.27 & $\mathrm{C}_{5} \mathrm{H}_{4} \mathrm{~N}_{4} \mathrm{O}_{2}^{-}$ & $1.3 \times 10^{3}$ & Xanthine & Yes \\
\hline \multirow[t]{2}{*}{155.069} & -1.45 & $\mathrm{C}_{6} \mathrm{H}_{9} \mathrm{~N}_{3} \mathrm{O}_{2}^{+}$ & $9.2 \times 10^{3}$ & Histidine & Yes \\
\hline & -0.41 & $\mathrm{C}_{6} \mathrm{H}_{9} \mathrm{~N}_{3} \mathrm{O}_{2}^{-}$ & $1.0 \times 10^{4}$ & & Yes \\
\hline \multirow[t]{2}{*}{156.065} & -0.74 & $\mathrm{C}_{5} \mathrm{H}_{8} \mathrm{~N}_{4} \mathrm{O}_{2}^{+}$ & $1.2 \times 10^{4}$ & 1,2,4-triazole-3-alanine & Yes \\
\hline & -0.04 & $\mathrm{C}_{5} \mathrm{H}_{8} \mathrm{~N}_{4} \mathrm{O}_{2}^{-}$ & $1.4 \times 10^{4}$ & & Yes \\
\hline 160.048 & 0.57 & $\mathrm{C}_{5} \mathrm{H}_{8} \mathrm{~N}_{2} \mathrm{O}_{4}^{-}$ & $4.1 \times 10^{3}$ & Thymine glycol & No \\
\hline 167.069 & -0.68 & $\mathrm{C}_{7} \mathrm{H}_{9} \mathrm{~N}_{3} \mathrm{O}_{2}^{-}$ & $6.4 \times 10^{3}$ & $\beta$-pyrazinyl-L-alanine & Yes \\
\hline \multirow[t]{2}{*}{169.085} & 0.18 & $\mathrm{C}_{7} \mathrm{H}_{11} \mathrm{~N}_{3} \mathrm{O}_{2}^{+}$ & $1.4 \times 10^{4}$ & 3-methylhistidine & Yes \\
\hline & -0.78 & $\mathrm{C}_{7} \mathrm{H}_{11} \mathrm{~N}_{3} \mathrm{O}_{2}^{-}$ & $6.0 \times 10^{3}$ & & Yes \\
\hline 171.064 & 0.15 & $\mathrm{C}_{6} \mathrm{H}_{9} \mathrm{~N}_{3} \mathrm{O}_{3}^{-}$ & $3.2 \times 10^{3}$ & $\beta$-hydroxyhistidine & Yes \\
\hline \multirow[t]{2}{*}{172.096} & 0.05 & $\mathrm{C}_{6} \mathrm{H}_{12} \mathrm{~N}_{4} \mathrm{O}_{2}^{+}$ & $2.7 \times 10^{4}$ & Iminoarginine & Yes \\
\hline & 0.55 & $\mathrm{C}_{6} \mathrm{H}_{12} \mathrm{~N}_{4} \mathrm{O}_{2}^{-}$ & $1.2 \times 10^{3}$ & & No \\
\hline \multirow[t]{2}{*}{182.080} & 0.72 & $\mathrm{C}_{7} \mathrm{H}_{10} \mathrm{~N}_{4} \mathrm{O}_{2}^{+}$ & $1.8 \times 10^{4}$ & Lathyrine & Yes \\
\hline & 0.54 & $\mathrm{C}_{7} \mathrm{H}_{10} \mathrm{~N}_{4} \mathrm{O}_{2}^{-}$ & $1.4 \times 10^{4}$ & & Yes \\
\hline 193.074 & 0.32 & $\mathrm{C}_{10} \mathrm{H}_{11} \mathrm{NO}^{-}$ & $1.3 \times 10^{3}$ & Phenylglycine, m-acetyl & No \\
\hline 206.080 & 0.76 & $\mathrm{C}_{9} \mathrm{H}_{10} \mathrm{~N}_{4} \mathrm{O}_{2}^{-}$ & $6.2 \times 10^{3}$ & Benzotriazolylalanine & Yes \\
\hline 226.107 & -0.26 & $\mathrm{C}_{9} \mathrm{H}_{14} \mathrm{~N}_{4} \mathrm{O}_{3}^{-}$ & $2.8 \times 10^{3}$ & Alanylhistidine & Yes \\
\hline 246.100 & -1.85 & $\mathrm{C}_{13} \mathrm{H}_{14} \mathrm{~N}_{2} \mathrm{O}_{3}^{-}$ & $1.6 \times 10^{3}$ & Acetyltryptophan & No \\
\hline
\end{tabular}

Note. The final column notes whether this formula is seen in Titan sample produced with the PHAZER set-up under CO-free conditions.

versus $\mathrm{O} / \mathrm{C}$ ratios of the identified molecules, which are widely used to understand the structures of complex organic matter on Earth (e.g., Kim et al., 2003). We separate the molecular ratios by whether they were seen in positive or negative ion mode. The difference in clustering between ion polarities clearly demonstrates that both are needed to understand the full chemical nature of the tholin's soluble fraction, which is frequently only a subset of the global molecular complexity (Maillard et al., 2018). Strongly oxygenated molecules clearly favor negative ionization while more heavily nitrogenated molecules increase in positive ionization mode. In H/C versus N/C space, we compare our results to PHAZER Titan tholin produced without CO. The Titan van Krevelen diagrams use molecular assignments performed with slightly different software, Attributor (Orthous-Daunay et al., 2020), but which has been benchmarked against $i d m o l$ and reliably produces comparable results at masses $m / z \leq 300$ (Bonnet et al., 2013; Hörst, 2011).

The Triton tholin reach high $\mathrm{O} / \mathrm{C}$ ratios across all $\mathrm{H} / \mathrm{C}$ ratios. Oxygen species are always more prevalent in the negative ionization data and consequently reach larger O/C ratios. We also show in Figure 6 an empty van Krevelen diagram that shows the regions in which particular molecular functional groups fall, for reference. The Triton tholin distinctly cluster above an $\mathrm{H} / \mathrm{C}$ of 1.0 , the demarcation of oxidation reactions and more highly 

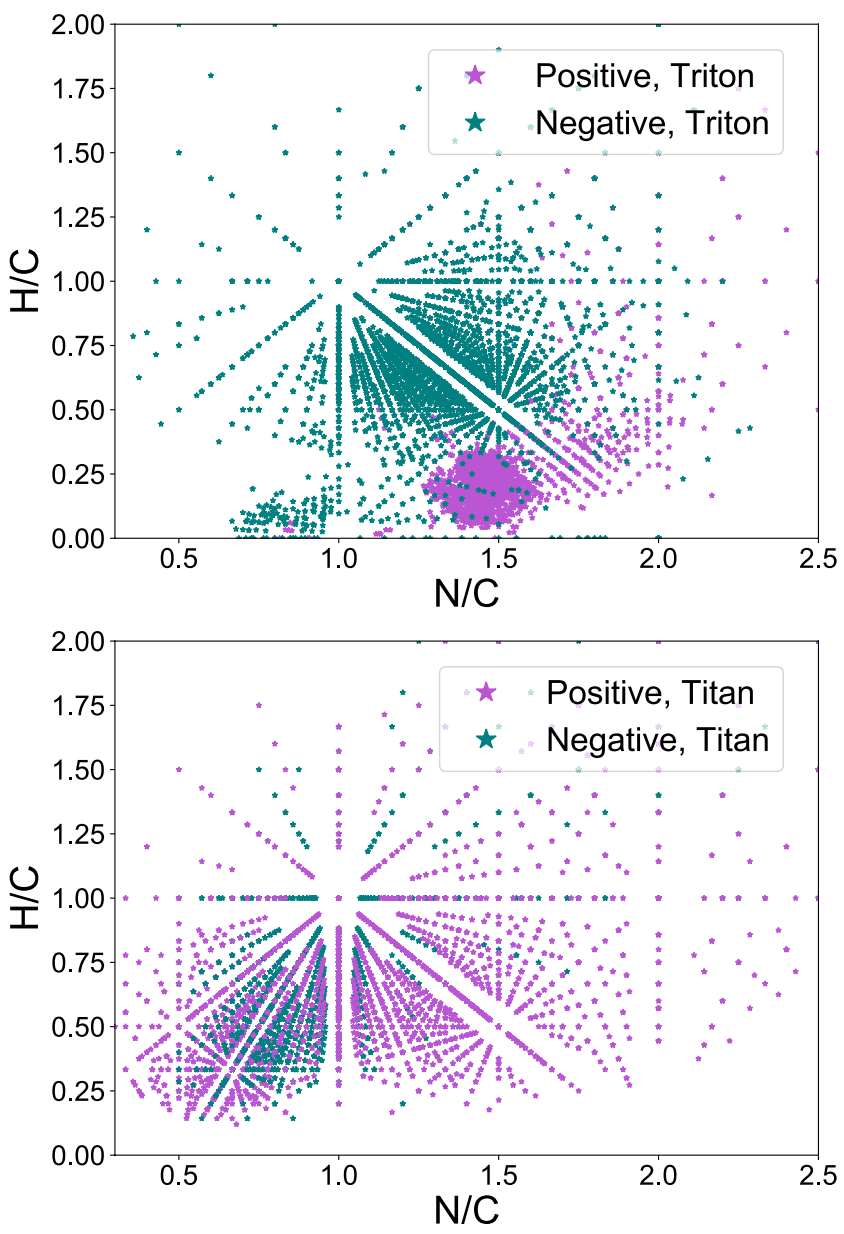

Figure 5. van Krevelen diagrams of the PHAZER Triton haze analogs (top) and PHAZER Titan haze analogs (bottom) in terms of H/C versus N/C, as is common in other tholin studies. The molecular character clearly differs between negative and positive ionizations, underscoring the need for both to understand the sample's chemistry. The nitrogen incorporation also clearly differs between the Titan sample and the Triton sample produced from a gas mix with $\mathrm{CO}$ dominant over $\mathrm{CH}_{4}$. unsaturated species. From the elemental analysis, however, we know that the overall hydrogen content is comparable between the Triton and Titan samples, reiterating our previous interpretation that oxygen is incorporated into the solid at the expense of carbon. The Triton tholin exhibit a shift away from simple unsaturated hydrocarbons to long chain carboxylic acids (fatty acids), bounded by $\mathrm{H} / \mathrm{C}$ ratios of $1.0-2.0$ and an $\mathrm{O} / \mathrm{C}$ ratio $\leq 0.4$. Additionally, the Triton tholin displays a large clustering of molecules consistent with amino acid and peptide-like species $(1.0 \geq \mathrm{H} / \mathrm{C} \leq 2.0 ; 0.2 \geq \mathrm{O} / \mathrm{C} \leq 0.8)$. Finally, the Triton tholin, due to its increased oxidation, shows greater clustering above $\mathrm{H} / \mathrm{C}$ of 1.5 and $\mathrm{O} / \mathrm{C}$ of 0.8 , where carbohydrate species fall on van Krevelen diagrams (Ruf et al., 2018).

In $\mathrm{H} / \mathrm{C}$ versus N/C space, the Titan tholin is relatively similar between positive and negative ionization, but the Triton tholin is dramatically different. While the negatively ionized molecules from the Triton tholin are qualitatively similar to Titan in this visualization, the nitrogenation of the Triton tholin is clearly enhanced above that of the Titan tholin as seen in the intense clustering of molecules above an N/C of 1.0. These results are also in strong contrast to the results of Jovanović et al. (2020), who studied varying CO-containing $\mathrm{N}_{2}-\mathrm{CH}_{4}$ mixtures for Pluto atmospheric composition. Jovanović et al. (2020) found, like with our Titan tholin, a preference for molecules with N/C ratios less than one, suggesting that oxygen is incorporated into the solid at the expense of nitrogen. As discussed in our elemental analysis results, we find instead that carbon depletion is associated with the addition of oxygen atoms into the tholin while nitrogen comparatively increases. These results strongly suggest an alternative chemical pathway is at work in the production of the solids when $\mathrm{CO}$ gas $\geq \mathrm{CH}_{4}$. The shift to a more oxidizing atmosphere increasing nitrogen fixation has precedence in early Earth studies with $\mathrm{CO}_{2}$ and $\mathrm{O}_{2}$ (Gavilan et al., 2018; Hörst, He, Ugelow, et al., 2018). Moreover, unlike our PHAZER Titan tholin, the positively ionized molecules in our PHAZER Triton tholin are remarkably tightly confined between N/C ratios of 1.2 and 1.5 and $\mathrm{H} / \mathrm{C}$ ratios of $0-0.5$. Combined with the increase in unsaturation and oxidation, this tight clustering is likely demonstrating that hydrogen atoms preferentially bond to nitrogen and oxygen atoms. As postulated in Hörst and Tolbert (2014) and He et al. (2017), these reactions may occur due to oxygen radicals from $\mathrm{CO}$ efficiently removing hydrogen atoms and molecules from $\mathrm{CH}_{4}$ along the pathway to solid haze formation.

\subsection{Transmission and Reflectance Spectra of the Triton Tholin}

\subsubsection{Functional Groups From VIS to NIR}

We show in Figure 7 the transmittance and reflectance spectra of the Triton tholin from visible to near-infrared wavelengths, $0.4-5 \mu \mathrm{m}\left(25,000\right.$ to $\left.2,000 \mathrm{~cm}^{-1}\right)$. In visible wavelengths, the most noticeable characteristic of the tholin is its sharp downward slope from $15,000 \mathrm{~cm}^{-1}$ in transmission, which is observable to the eye as the tholin's brown-ish orange color, well known from Titan tholin studies. In the near-IR, we attribute the variety of features observed to a combination of $\mathrm{N}-\mathrm{H}, \mathrm{C}-\mathrm{H}, \mathrm{C} \equiv \mathrm{N}, \mathrm{C}=\mathrm{C}$, and $\mathrm{O}-\mathrm{H}$ bonds. Amine $\left(\mathrm{N}-\mathrm{H}\right.$ and $\left.\mathrm{N}-\mathrm{H}_{2}\right)$ features are well described in Titan and Pluto tholin in the $3,500 \mathrm{~cm}^{-1}$ to $3,000 \mathrm{~cm}^{-1}$ regions (e.g., Imanaka et al., 2004; Jovanović et al., 2020), but given the prevalence of $\mathrm{O}$ species identified in the mass spectral data and combustion analysis, we assign a subset of these features tentatively to $\mathrm{O}-\mathrm{H}$ alcohol bonds in addition to amines. There is slight deepening and broadening of the amine feature out to $2,500 \mathrm{~cm}^{-1}$, which could derive from carboxylic acid species with $\mathrm{O}-\mathrm{H}$ bonds, which would be consistent with the clustering we describe above in the van Krevelen diagrams. Around the $3,000 \mathrm{~cm}^{-1}$ region, we identify a feature consistent with various $\mathrm{C}-\mathrm{H}$ bonds, namely alkenes and alkanes. Between 2,300 and 2,000 $\mathrm{cm}^{-1}$, we see some combination of nitrile, alkyne, and imine functional groups. Overall, this plethora of features underscores the complex nature of tholin. We provide a complete list of 

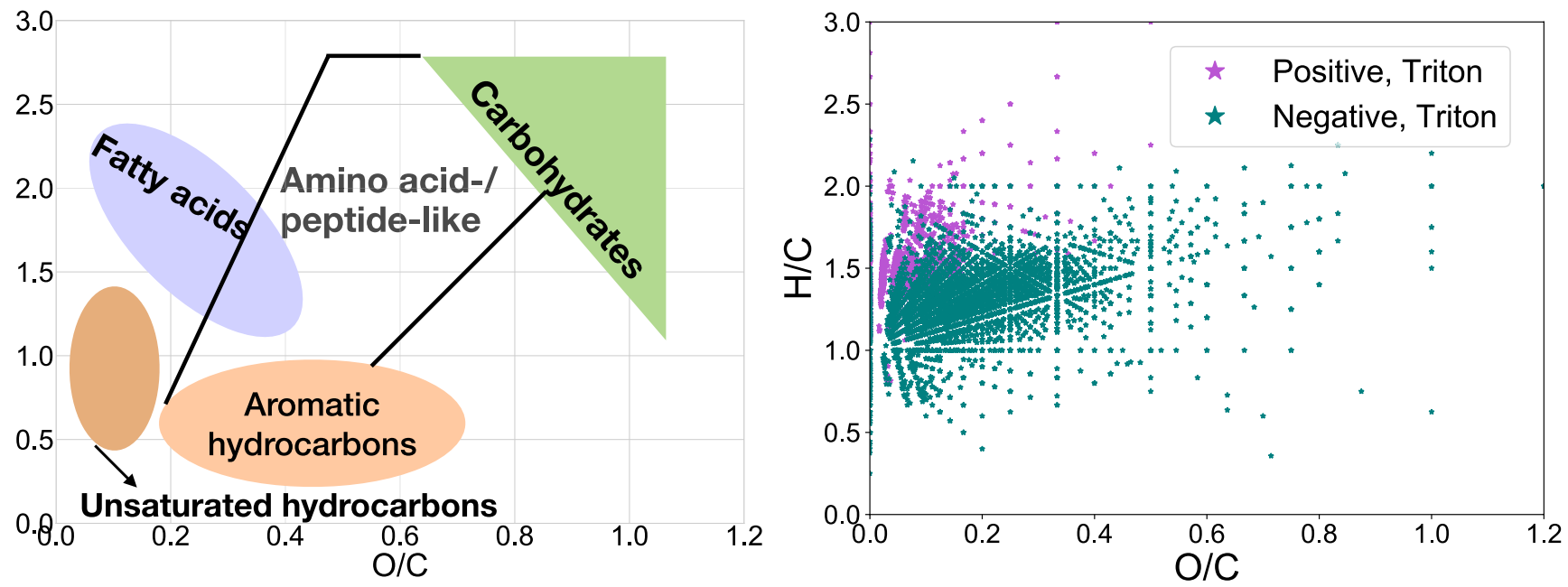

Figure 6. Left: An empty van Krevelen diagram showing H/C versus $\mathrm{O} / \mathrm{C}$ with shaded, labeled regions denoting where particular molecular functional groups tend to cluster, following Ruf et al. (2018). Right: van Krevelen in terms of H/C versus O/C for our Triton sample. The Triton sample clusters strongly in the regions for fatty acids and amino acid-like molecular groups.

potential attributions of the spectra in Table 2. We also observe some weak features likely indicative of adsorption from ambient atmosphere or residuals in the spectrometer $(\sim 1 \mathrm{hPa})$ when the sample is briefly exposed to air (water at 3,900-3,700 $\mathrm{cm}^{-1}$ and carbon dioxide at 2,370 $\mathrm{cm}^{-1}$ ), which is also seen in Pluto-like tholin (Jovanović et al., 2020).

Unfortunately, due to our quartz substrate which strongly absorbs in the mid-IR, we are not sensitive to wavelengths beyond $5 \mu \mathrm{m}\left(2,000 \mathrm{~cm}^{-1}\right)$, where carbonyl groups $(\mathrm{C}=\mathrm{O})$ have prominent spectral features. While other substrates $(\mathrm{KBr}, \mathrm{Si}$, etc.) would enable better mid-IR spectroscopy, these materials still require vetting for chemical non-reactivity in our experimental set-up, which is the subject of future work. With quartz, the only $\mathrm{O}$ bonds we can readily detect are $\mathrm{O}-\mathrm{H}$ bonds, which could be alcohols or carboxylic acids. Both of these have clusters in the relevant regions of our van Krevelen diagrams (Figure 6), but considerably more molecules fall in the carboxylic acid region (where the line with intercept at $\mathrm{H} / \mathrm{C}=2.0$ has a slope of -1 ) than in the alcohol region

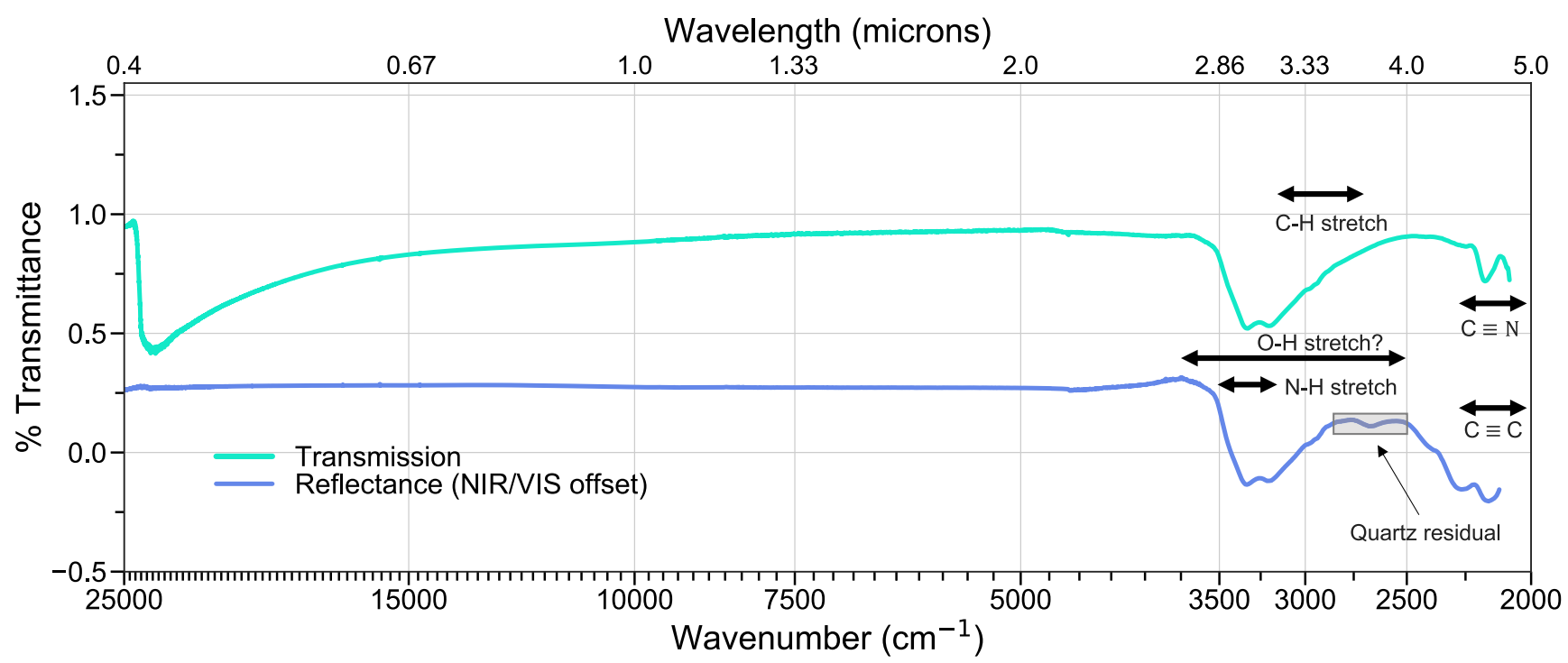

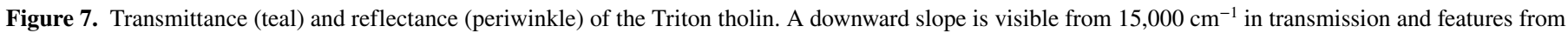

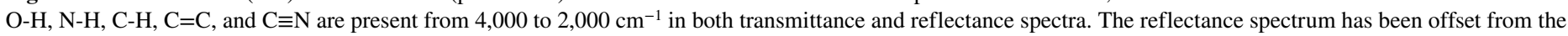

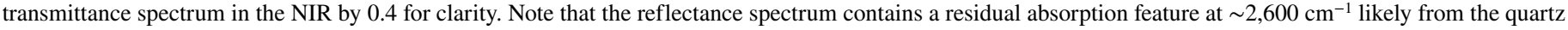
substrate. 
Table 2

Spectral Features and Corresponding Functional Groups Identified in the Triton Sample

\begin{tabular}{|c|c|c|c|}
\hline Frequency $\left(\mathrm{cm}^{-1}\right)$ & Wavelength $(\mu \mathrm{m})$ & Potential functional group (bond, type) & Feature (extent, strength) \\
\hline $3,900-3,700$ & $2.56-2.7$ & $\mathrm{H}_{2} \mathrm{O}$ adsorption (contaminant) & Broad, weak \\
\hline $3,560-2,889$ & $2.8-3.46$ & $\mathrm{~N}-\mathrm{H}$ stretching (amine) & Broad, strong \\
\hline 3,337 & 2.99 & $-\mathrm{NH}_{2}$ asymmetric stretching, $-\mathrm{NH}-$ stretching (amines) & Sharp, strong \\
\hline 3,260 & 3.07 & N-H stretching (amine) & Sharp, strong \\
\hline $3,200-3,190$ & $3.12-3.13$ & $-\mathrm{NH}-$ stretching, overtone of $-\mathrm{NH}_{2}$ bending (amines) & Sharp, strong \\
\hline $3,500-2,500$ & $2.86-4.0$ & O-H stretching (alcohols)?, O-H stretching (carboxylic acids)? & Broad, strong \\
\hline $3,004-2,906$ & $3.32-3.44$ & $-\mathrm{CH}_{3},-\mathrm{CH}_{2}-$ asymmetric stretching (alkenes, alkanes) & Shoulder, weak \\
\hline 2,370 & 4.21 & $\mathrm{CO}_{2}$ residual (contaminant) & Shoulder \\
\hline $2,340-2,215$ & $4.27-4.51$ & $-\mathrm{C} \equiv \mathrm{N}$ stretching (nitrile) & Broad, weak \\
\hline 2,210 & 4.52 & $\mathrm{C} \equiv \mathrm{C}$ stretching (alkyne), $-\mathrm{C} \equiv \mathrm{N}$ stretching (aromatic nitrile) & Sharp, strong \\
\hline 2,145 & 4.66 & $-\mathrm{C} \equiv \mathrm{N}$ stretching (nitrile), $-\mathrm{N}=\mathrm{C}=\mathrm{N}-$ (carbodiimide), $\mathrm{CO}$ fundamental & Shoulder \\
\hline
\end{tabular}

(where the line with intercept at $\mathrm{H} / \mathrm{C}=2.0$ has a slope of 0 ). Overall, the spectral features we are able to observe at wavenumbers above $2,000 \mathrm{~cm}^{-1}$ are consistent with the functional groups we identify from HRMS.

\subsubsection{Comparison to Other Tholin Spectra}

We compare our measured transmission spectrum of Triton tholin to a collection of literature-reported values for tholin generated from nitrogen/methane atmospheres. We do not report every single tholin experiment to date, but select experiments which contain $\mathrm{CO}$ or have a Triton-focus. We also generally include only those data published originally as spectra, rather than including each set of published optical constants. We make an exception for the work of Khare et al. due to their widespread use and for Jovanović et al. (2021) as their Pluto-like experimental mixtures are otherwise the most similar to our Triton mixture. The Titan optical constants of Khare et al. (1984), the Triton-specific optical constants of Khare et al. (1994), and the Pluto-like optical constants of Jovanović et al. (2021) provide the complex refractive indices, $n$ and $k$, which they derive from their measured spectra. To convert these values back to spectra for comparison here, we follow the method from Khare et al. (1984) given by

$$
\begin{gathered}
R=\frac{(n-1)^{2}+k^{2}}{(n+1)^{2}+k^{2}} \\
T=(1-R) e^{\lambda / 4 t \pi} e^{-k}
\end{gathered}
$$

where $R$ and $T$ are the reflectance and transmittance, respectively, $n$ is the real refractive index, $k$ is the imaginary refractive index, $\lambda$ is the wavelength in microns, and $t$ is the film thickness, which in the region of interest $(0.4-5 \mu \mathrm{m})$ is reported in each publication. We affirm that the reflectance spectrum we derive from the given complex refractive indices in Khare et al. (1984) is identical to their Figure 5. The full set of $n$ and $k$ from Khare et al. (1984) and Jovanović et al. (2021) are available in the literature, but for completeness we include these values from Khare et al. (1994) in Table A1 of Appendix A.

A general summary of the experimental conditions used to generate each set of tholin spectra discussed here is found in Table 3. Note that a more detailed discussion of most experimental conditions, as well as the wider range of experiments used to measure optical constants of (Titan) tholin, can be found in the review by Brassé et al. (2015).

We present a comparison of our Triton tholin transmittance spectra, PHAZER Titan transmittance spectra (He et al., 2022), and the other experimental data to which we compare our results in Figure 8. The PHAZER Titan tholin spectra has wider absorption at the blue visible edge $(0.4 \mu \mathrm{m})$ compared to our Triton spectra, consistent with the slightly bluer cast of Triton's haze compared to Titan's, which is known from photometry (Hillier et al., 2021). The most noticeable similarity amongst all the spectra is the broad feature between 3,600 and $3,200 \mathrm{~cm}^{-1}$, which results from N-H bonds in the form of primary and secondary amines. The most overall similar spectra are the PHAZER Titan (He et al., 2022) and Triton (this work) spectra, which is not surprising given 
Table 3

Summary of Experimental Conditions of the Tholin Spectra Presented in Figure 8

\begin{tabular}{|c|c|c|c|c|}
\hline Reference/Body & Gas mixture & Temperature & Pressure & Energy source \\
\hline $\begin{array}{l}\text { This work } \\
\text { Triton }\end{array}$ & $\begin{array}{l}99.3 \% \mathrm{~N}_{2} \\
0.5 \% \mathrm{CO} \\
0.2 \% \mathrm{CH}_{4}\end{array}$ & $90 \mathrm{~K}$ & $\sim 1$ mbar & $\begin{array}{l}\text { AC plasma } \\
\text { discharge }\end{array}$ \\
\hline $\begin{array}{l}\text { He et al. (2022) } \\
\text { Titan }\end{array}$ & $\begin{array}{l}95 \% \mathrm{~N}_{2} \\
5 \% \mathrm{CH}_{4}\end{array}$ & $90 \mathrm{~K}$ & $\sim 1 \mathrm{mbar}$ & $\begin{array}{l}\text { AC plasma } \\
\text { discharge }\end{array}$ \\
\hline $\begin{array}{l}\text { McDonald et al. (1994) } \\
\text { Triton }\end{array}$ & $\begin{array}{l}99.9 \% \mathrm{~N}_{2} \\
0.1 \% \mathrm{CH}_{4}\end{array}$ & Room temperature (294 K) & $\sim 1 \mathrm{mbar}$ & RF ICP discharge \\
\hline $\begin{array}{l}\text { Tran et al. (2008) } \\
\text { Titan }\end{array}$ & $\begin{array}{c}98 \% \mathrm{~N}_{2} \\
1.8 \% \mathrm{CH}_{4} \\
0.2 \% \mathrm{H}_{2} \\
400 \mathrm{ppm} \mathrm{C}_{2} \mathrm{H}_{2} \\
300 \mathrm{ppm} \mathrm{C}_{2} \mathrm{H}_{4} \\
20 \mathrm{ppm} \mathrm{HC}_{3} \mathrm{~N} \\
0.3 \% \mathrm{CO}\end{array}$ & $297 \mathrm{~K}$ & $900 \mathrm{mbar}$ & Mercury lamp \\
\hline $\begin{array}{l}\text { Jovanović et al. (2021) } \\
\text { Pluto, } H(<350 \mathrm{~km})\end{array}$ & $\begin{array}{c}99.5 \% \mathrm{~N}_{2} \\
0.5 \% \mathrm{CH}_{4} \\
500 \mathrm{ppm} \mathrm{CO}\end{array}$ & Room temperature (294 K) & $\sim 1 \mathrm{mbar}$ & $\begin{array}{l}\text { RF CCP } \\
\text { discharge }\end{array}$ \\
\hline $\begin{array}{l}\text { Jovanović et al. }(2020,2021) \\
\text { Pluto, } 400 \mathrm{~km}\end{array}$ & $\begin{array}{c}99 \% \mathrm{~N}_{2} \\
1 \% \mathrm{CH}_{4} \\
500 \mathrm{ppm} \mathrm{CO}\end{array}$ & Room temperature (294 K) & $\sim 1$ mbar & $\begin{array}{l}\text { RF CCP } \\
\text { discharge }\end{array}$ \\
\hline $\begin{array}{l}\text { Jovanović et al. (2021) } \\
\text { Pluto, } 650 \mathrm{~km}\end{array}$ & $\begin{array}{c}95 \% \mathrm{~N}_{2} \\
5 \% \mathrm{CH}_{4} \\
500 \mathrm{ppm} \mathrm{CO}\end{array}$ & Room temperature (294 K) & $\sim 1 \mathrm{mbar}$ & $\begin{array}{l}\text { RF CCP } \\
\text { discharge }\end{array}$ \\
\hline $\begin{array}{l}\text { Imanaka et al. (2004) } \\
\text { Titan }\end{array}$ & $\begin{array}{l}90 \% \mathrm{~N}_{2} \\
10 \% \mathrm{CH}_{4}\end{array}$ & Room temperature (294 K) & 0.67 mbar & RF ICP discharge \\
\hline $\begin{array}{l}\text { Khare et al. (1984) } \\
\text { Titan }\end{array}$ & $\begin{array}{l}90 \% \mathrm{~N}_{2} \\
10 \% \mathrm{CH}_{4}\end{array}$ & Room temperature (294 K) & 0.2 mbar & $\begin{array}{l}\text { DC plasma } \\
\text { discharge }\end{array}$ \\
\hline $\begin{array}{l}\text { Khare et al. (1994) } \\
\text { Triton }\end{array}$ & $\begin{array}{l}99.9 \% \mathrm{~N}_{2} \\
0.1 \% \mathrm{CH}_{4}\end{array}$ & Room temperature (294 K) & 0.86 mbar & $\begin{array}{l}\text { DC plasma } \\
\text { discharge }\end{array}$ \\
\hline
\end{tabular}

Note. AC, Alternating current; RF ICP, Radio frequency inductively coupled plasma; RF CCP, Radio frequency capacitively coupled plasma; DC, Direct current.

the known effect of different experimental set-ups on resulting tholin properties (e.g., Brassé et al., 2015; Cable et al., 2012). Our Triton tholin spectra have broad, wide features in the 3,600-3,200 $\mathrm{cm}^{-1}$ region, which could also be due to the inclusion of oxygen in the chemical structure of the tholin, which could add $\mathrm{O}-\mathrm{H}$ stretching from alcohols. This broadening extends to nearly $2,500 \mathrm{~cm}^{-1}$ and is apparent even in comparison to the PHAZER Titan data, which has absorption neither as wide nor as deep at these wavelengths.

The second commonality to most spectra (except McDonald et al. [1994] and Khare et al. [1994] likely due to their very low starting methane mixing ratios) is a narrower feature at $\sim 2,200 \mathrm{~cm}^{-1}(4.5 \mu \mathrm{m})$. This absorption is likely from some combination of $\mathrm{C} \equiv \mathrm{C}$ (alkyne) or $\mathrm{C} \equiv \mathrm{N}$ (nitrile) bonds. While our Triton spectrum and Jovanović et al. (2020)'s Pluto spectrum have similarly wide and deep features here, the PHAZER Titan spectrum (He et al., 2022) is markedly narrower and deeper at this feature, suggesting a different balance of alkynes to nitriles than that of the Pluto and Triton tholin. 

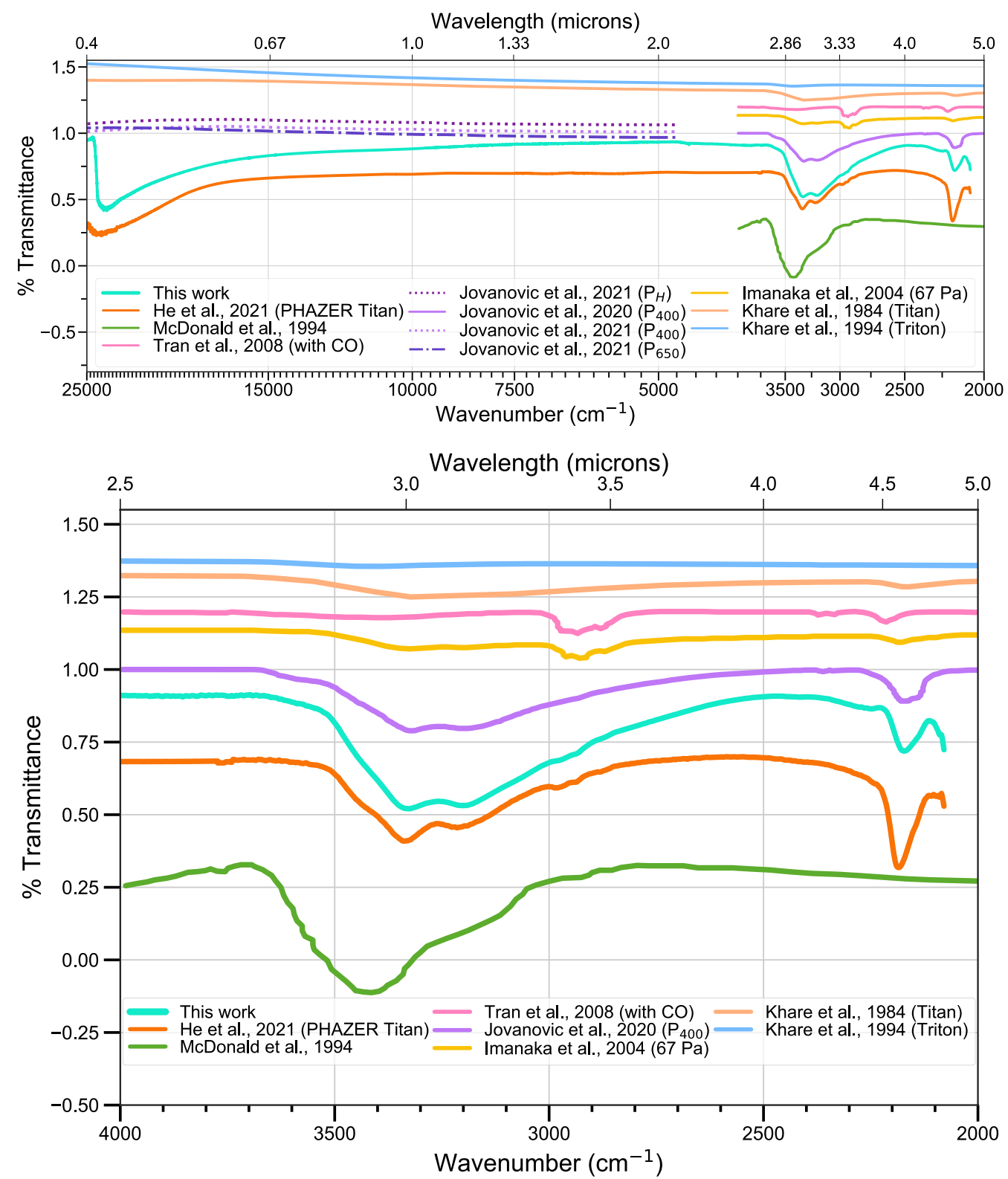

Figure 8. Top: Transmittance spectra of various tholins produced from $\mathrm{N}_{2}-\mathrm{CH}_{4}$ mixtures. The full details of each experiment can be found in Table 3. Some features are present in all tholin. In the Triton tholin of this work, we attribute some of the broadening and additional absorption between 3,500 and $2,500 \mathrm{~cm}^{-1}$ to be due uniquely to $\mathrm{O}-\mathrm{H}$ bonds. These bonds likely result from $\mathrm{CO}>\mathrm{CH}_{4}$ in the initial gas mixture. All spectra have been offset vertically for clarity. Bottom: As above with emphasis on the $4,000 \mathrm{~cm}^{-1}$ to $2,000 \mathrm{~cm}^{-1}$ region.

Our Triton tholin, PHAZER Titan tholin (He et al., 2022), Imanaka et al. (2004) tholin, and Tran et al. (2008) tholin all have features at $\sim 3,000 \mathrm{~cm}^{-1}$, which are indicative of $\mathrm{C}-\mathrm{H}$ bonds in the form of alkenes or alkanes. These features are notably absent in the spectra of Jovanović et al. (2020). He et al. (2022), Imanaka et al. (2004), and Tran et al. (2008) all produced tholin from much higher methane mixing ratios (or in the case of Tran et al., higher mixing ratios of other hydrocarbon species) than either Jovanović et al. (2020) or our Triton tholin. This suggests the additional absorption we see in the Triton tholin spectra in this region $\left(\sim 3,000\right.$ to $\left.2,500 \mathrm{~cm}^{-1}\right) \mathrm{could}^{-}$ derive from additional $\mathrm{O}-\mathrm{H}$ bonds from alcohols and carboxylic acids that are not present in the Pluto composition tholin of Jovanović et al. (2020) because of the smaller amount of CO in their starting gas mixture. In a follow-up study, Jovanović et al. (2021) measured optical constants for Pluto analog aerosols as well, varying the $\mathrm{CH}_{4}$ mixing ratio but holding $\mathrm{CO}$ steady. In effect, this explored the importance of $\mathrm{CO}$ as it competes with 
$\mathrm{CH}_{4}$. In agreement with our results here, their optical constants results show that $\mathrm{N}$ - and O-bearing molecules increase and generate additional absorption in the visible and near-IR with lower methane mixing ratios. Their study extended into the UV as well, finding that this absorption due to $\mathrm{N}$ and $\mathrm{O}$ molecules also affects shorter wavelengths (Jovanović et al., 2021).

We also note that many of the spectra to which we compare have their strongest identifying features at slightly longer wavelengths and into the fingerprint region, from roughly $5-15 \mu \mathrm{m}\left(1,700-700 \mathrm{~cm}^{-1}\right)$. This region is highly complex because of the multitude of features found there, but it can also provide much needed context to the VIS-NIR wavelength spectra explored here, particularly regarding the oxygen-bonding environment of tholin or haze particles. Carbonyl $(\mathrm{C}=\mathrm{O})$ species including aldehydes, ketones, esters, amides, and carboxylic acids all have prominent peaks from 1,800 to $1,600 \mathrm{~cm}^{-1}(5.55-6.25 \mu \mathrm{m})$, and in fact have been observed in other studies with relatively larger CO starting fractions (Tran et al., 2008). Additionally, a study which examined the effect of $\mathrm{CO}_{2}$ in $\mathrm{N}_{2}-\mathrm{CH}_{4}$ gas mixtures also found significant enhancement in both $\mathrm{N}$ - and O-bearing functional groups as $\mathrm{CO}_{2}$ increased (Gavilan et al., 2018). This study examined both shorter (UV, down to $0.13 \mu \mathrm{m}$ ) and longer (MIR, 6-10 $\mu \mathrm{m}$ ) wavelengths, reitering the importance of wide wavelength coverage in understanding increasingly oxidized aerosols.

\section{Discussion}

\subsection{Comparison to Existing Observations of Triton}

\subsubsection{Voyager 2 Atmospheric Observations}

In situ observations of Triton's atmosphere are mainly limited to visible and UV photometry rather than spectra, preventing a true direct comparison of our experimental tholin results. Voyager 2 imaging and visible photometry observations of Triton's atmosphere suggest optically thin hazes (Smith et al., 1989), with scattering optical depths between 0.001-0.01 that increase with shorter wavelengths (Pollack et al., 1990; Rages \& Pollack, 1992) and particle sizes of around 100-200 nm (Pollack et al., 1990; Rages \& Pollack, 1992). UV occultation data from Voyager 2 suggests significantly higher (0.024) scattering optical depths, reiterating the wavelength dependent nature of the haze scattering and suggesting even smaller particles $(30 \mathrm{~nm})$ in the Rayleigh regime (Herbert \& Sandel, 1991). At visible wavelengths, the haze is strongly forward scattering and requires particles larger than $200 \mathrm{~nm}$ (Hillier et al., 1991). To meet these observational signatures of higher scattering in the blue (small particles) and large forward scattering (large particles) simultaneously, the hazes have been suggested to be fractal aggregates (Lavvas et al., 2021; Ohno et al., 2021). Aggregates also explain the haze properties of both Titan (Tomasko et al., 2008) and Pluto (Cheng et al., 2017; Gao et al., 2017; Gladstone et al., 2016; Lavvas et al., 2021), though the fractal dimension and growth rate is not settled (Kutsop et al., 2021). This range of particle sizes well fits with those we produce in the PHAZER chamber (e.g., He et al., 2017, 2018), though these particle sizes may in part result from the physical size of the chamber itself, which inhibits further growth. Material characterization of PHAZER Titan tholin particles suggests, however, that the necessary particle size and shape to match observations is easily achieved through coagulation (Yu et al., 2017). More generally, tholin particles produced in a variety of setups seem to easily coagulate and form aggregates (Cable et al., 2012).

\subsubsection{Seasonal Changes From Space- and Ground-Based Observations}

Ground-based observations were responsible for the initial confirmed detection of $\mathrm{CO}$ in Triton's atmosphere (Lellouch et al., 2010). However, earlier Hubble Space Telescope (HST) data using the Space Telescope Imaging Spectrograph (STIS) also provided upper limits on CO that differed from the initial Voyager 2 data and suggested strong surface absorption at UV wavelengths, which could be attributable to photochemical products from the atmosphere settling to the surface (Krasnopolsky \& Cruikshank, 1995; Stern et al., 1995). Both the ground- and space-based data indicate that CO levels in the atmosphere can change over seasonal timescales. Further HST STIS observations showed that Triton's UV albedo brightened between the Voyager 2 flyby and 1999, evidence of ongoing seasonal cycling in the form of volatile transport between the surface and atmosphere or plume activity (Young \& Stern, 2001). Later HST and ground-based observations continued to find surface reflectivity changes in the UV, as well as in visible and NIR wavelengths which probe methane abundances, suggestive of continued seasonal volatile transport on sub-decadal timescales (Bauer et al., 2010; Buratti et al., 2011). Additionally, both thermal and pressure changes have been observed from ground-based solar occultations (Elliot et al., 1998; Olkin et al., 1997), demonstrating that the atmosphere undergoes substantial interaction with the surface. The 
surface is predominately $\mathrm{N}_{2}$ ice, with contributions from diluted $\mathrm{CO}$ ice (Merlin et al., 2018). This CO ice and $\mathrm{N}_{2}$ ice experience greater longitudinal and temporal variation than does $\mathrm{CH}_{4}$ ice present on the surface (Grundy et al., 2002, 2010), including over diurnal timescales (Holler et al., 2016).

Given our results regarding the importance of the exact $\mathrm{CO} / \mathrm{CH}_{4}$ mixing ratio in determining both the nitrogenation and oxidation of haze particles, these observations suggest that large shifts can happen in the dominant haze chemistry over Triton's seasonal cycle, as may also be occurring on Pluto (Bertrand \& Forget, 2017). In their study of Pluto tholin optical constants, Jovanović et al. (2021) found that the real refractive index $n$ increased when the relative $\mathrm{CO}$ mixing ratio in the gas increased, and oxidation of the aerosols promoted both UV and visible absorption. Therefore, our experimental results combined with past observations suggests a complicated set of processes likely controls Triton's atmospheric and surface properties. Ices sublimate, change the overall atmospheric abundances, shift haze chemistry, and these hazes settle or condense out onto the surface, whereupon both ices and hazes can undergo additional alteration by cosmic rays and UV bombardment before potentially cycling back to the atmosphere again.

\subsection{Haze Formation and Ice Condensation}

In light of this active surface-atmospheric interaction, a growing body of literature suggests that ice condensation, be it of photochemical products directly or with them as condensate nuclei for other ices (Luspay-Kuti et al., 2017; Ohno et al., 2021), could play a large role in Triton's atmosphere. In fact, Lavvas et al. (2021) argues that Titan, Pluto, and Triton lie along a continuum of haze formation outcomes, with Titan at the "molecular growth" end and Triton on the "condensate growth" end, with Pluto in the middle. These results depend on models which simulate organic ice formation (Lavvas et al., 2021), but do not consider the implications of how photochemical haze formation may change due to the presence of $\mathrm{CO}$, which experimental studies have shown can be an important driver in haze formation and growth (He et al., 2017, 2018, 2020; Hörst, He, Lewis, et al., 2018; Hörst \& Tolbert, 2014). While our experiment simulates an atmosphere with $\mathrm{CO}>\mathrm{CH}_{4}$, as is present in Triton's atmosphere, we have increased their relative amounts compared to the background $\mathrm{N}_{2}$ in order to increase our tholin yield within a shorter timeframe. Our experiment used $0.5 \% \mathrm{CO}$ and $0.2 \% \mathrm{CH}_{4}$ while Lellouch et al. (2010) calculated upper limits of $0.18 \% \mathrm{CO}$ and $0.034 \% \mathrm{CH}_{4}$ in Triton's atmosphere.

While it may be tempting to interpret this as evidence for condensation processes over photochemical processes for the production of Triton's haze, many unknowns still exist, precluding a clear answer for the source of Triton's haze as of yet. Lower ratios (as is more reflective of the absolute abundances on Triton) can still generate substantial solid material photochemically. Previous work on $\mathrm{N}_{2}-\mathrm{CH}_{4}$ atmospheres has shown that, even with very low $(0.1 \%-0.01 \%)$ methane mixing ratios, many solid particles are still generated (Trainer et al., 2004), up to the same order in number and density of solids produced under more Titan-like methane mixing ratios (Hörst \& Tolbert, 2013). As discussed in Section 3.1, the role of the carbon source in haze production is not settled either, with varying amounts of $\mathrm{CO}$ and $\mathrm{CH}_{4}$ not decisively showing higher yields across different experimental set-ups. While Lavvas et al. (2021) argues that condensation of photochemical products occurs more quickly on Triton than molecular growth of solids can occur, these results rely on extrapolated nucleation and condensation rates for the materials in question. Our results, along with other laboratory measurements (see Section 3.1) suggests further study is warranted into the timescales of both processes for Triton, as well as on Pluto.

Future experimental work to simulate the uppermost reaches of Triton's atmosphere where haze formation initiates, without $\mathrm{CH}_{4}$, could help disentangle the relative roles of carbon monoxide versus methane in haze formation. Such work could isolate not only the relative importance of each in the chemical composition of the ultimate haze material, but also whether photochemical haze or ice condensates are more favorable for production of the haze observed in Triton's atmosphere. Moreover, UV lamp and plasma produced tholin are known to sometimes differ in composition and spectra, hinting at differing mechanisms for haze initiation in the ionosphere and the lower atmosphere (Cable et al., 2012; Trainer et al., 2006), which could also be investigated by future work with respect to Triton studies.

Prior comparisons arguing for ice condensates over photochemical products have also primarily used the optical constants of Khare et al. (1984) Titan tholin (e.g., Ohno et al., 2021). In the case of Pluto, more recent optical constants of purely photochemical Pluto tholin (from CO-containing mixtures) provide better agreements to existing New Horizons data of Pluto's surface (Jovanović et al., 2021), but there may still be a substantial contribution 
from condensed ices onto the hazes (see, e.g., Fayolle et al., 2021). Moreover, regardless of whether the particles are condensed ices or photochemical hazes that have settled to the surface, UV photons or cosmic ray bombardment could alter either set of particles, resulting in similar optical properties (e.g., Gudipati et al., 2013). Our results, which show increased nitrogenation and oxidation even over that observed by Jovanović et al. (2020, 2021), may further resolve observational discrepancies for Triton that do not necessarily require a substantial ice condensate component, or which could alter the behavior of hazes as potential condensate nuclei.

Further radiative transfer modeling has suggested that perhaps the redder Pluto and Titan hazes are more similar to each other in comparison to Triton, which is bluer. Such differences could be either chemical, perhaps due to higher $\mathrm{CO} / \mathrm{CH}_{4}$ mixing ratios as we have shown here, or physical, potentially due to the increased contribution from ice condensates in Triton's atmosphere (Hillier et al., 2021). The question of whether Triton's (and Pluto's) hazes are solids produced from condensation or molecular growth is far from settled. To pinpoint the cause of the similarities and differences between the hazes of these worlds, further measurements to obtain optical constants of the spectra we provide here, laboratory measurements of the formation and properties of heterogeneous hazeice condensate particles - across multiple haze chemistries, and further modeling studies are all required.

\subsection{Comparison to Other Experimental Results}

We demonstrate above that the formulas for a variety of astrobiologically interesting molecules (amino acids, nucleobases, and a simple sugar) are present in our tholin, and subsequent sedimentation of this putative haze material likely coats the icy surface of Triton, as also likely occurs on Pluto (Grundy et al., 2018; Protopapa et al., 2020). However, amino acids may be subject to further photolysis on icy surfaces, suggesting that they are rapidly destroyed unless this material quickly reaches the subsurface (Johnson et al., 2012), though Triton's $\sim 14 \mu$ bar atmosphere attenuates more UV radiation than does Europa (with an atmosphere of 1 picobar) which Johnson et al. (2012)'s experiment simulated. Complementing our atmospheric study, Triton surface ice chemistry by photolysis can also produce chemically complex material from $\mathrm{N}_{2}-\mathrm{CH}_{4}-\mathrm{CO}$ ice mixtures (Hodyss et al., 2011; Moore \& Hudson, 2003). The products of these surface ice experiments with $\mathrm{CO} / \mathrm{CH}_{4} \sim 1$ include many of the molecular species and spectral features we identify here, including carboxylic acids, alcohols, ketones, aldehydes, amines, and nitriles in addition to having similar nitrogen, carbon, and oxygen abundances overall (Materese et al., 2014, 2015). Additionally, potential interactions of these ices and tholin-like materials could further react with water ice deposits present on Triton's surface (Cruikshank et al., 2000) to generate molecules of additional prebiotic complexity and interest (Cruikshank et al., 2019). Given the seasonal cycling through sublimation and condensation of volatile ices combined with the products of photochemistry both in the atmosphere and on the surface, Triton holds great interest for future study.

\subsection{Future Triton Missions}

While ground- and space-based observations have furthered our understanding of Triton in the years since the Voyager 2 flyby (e.g., Bauer et al., 2010; Lellouch et al., 2010; Stansberry et al., 2015; Stern et al., 1995; Young \& Stern, 2001), in situ missions would dramatically improve our knowledge of the atmosphere, surface, and interior processes of the moon (Christophe et al., 2012; Fletcher et al., 2020; Hofstadter et al., 2019; Masters et al., 2014). Several proposed missions in various states of development would visit Triton, with complementary goals. Trident, a NASA Discovery class mission that was downselected as a finalist, though not ultimately chosen for flight in the Discovery 15 and 16 competition, would perform a single flyby of Triton (Prockter et al., 2019). The Neptune Odyssey mission concept, a NASA Flagship class orbiter and probe under study for the 2023 Planetary Science and Astrobiology Decadal Survey, would orbit the Neptune-Triton system with a four year prime mission and would perform $\sim$ monthly flybys of Triton itself (Rymer et al., 2020). Triton Hopper is a mission concept under study by the NASA Innovative Advanced Concepts (NIAC) program, which would act as a lander capable of producing its own propellant from surface ices in order to perform short flights across the surface (Landis et al., 2019; Oleson \& Landis, 2018).

Trident's major scientific goals would be to confirm the presence of a subsurface ocean and infer whether it interacts with the surface, sample the ionosphere, and perform repeated surface imaging to characterize its composition and geology (Prockter et al., 2019). To achieve these goals, Trident would be equipped with, among other instruments, a plasma spectrometer and a high-resolution infrared spectrometer with spectral range up to $5 \mu \mathrm{m}$. 
In the context of the study we have performed here, the Trident mission could clearly benefit our understanding of ionospheric processes driving haze formation by providing better constraints on the ion energies of the upper atmosphere. On Titan, for example, haze formation in the upper atmosphere is more strongly influenced by the Saturnian magnetosphere and solar EUV while longwave solar UV photons primarily dominate haze formation processes below $500 \mathrm{~km}$ (Lavvas et al., 2008). In laboratory measurements, plasma or spark discharge energy sources compared to UV lamps produce tholin which differs in composition and observable spectra (Cable et al., 2012), illustrating this dual haze formation process. A better understanding of the Triton ionosphere could similarly help guide future laboratory experiments and modeling efforts to describe the haze formation process for Triton specifically. Trident's infrared spectrometer would have the same range as the spectral measurements we have performed in this study (out to $5 \mu \mathrm{m}$ ) and would clearly advance our understanding of Triton's surface and atmosphere-surface interactions (Prockter et al., 2019). However, as we have shown above, the presence of $\mathrm{CO}$ as both a surface ice and and as a minor atmospheric component should induce significant oxygen chemistry. Such chemistry could be best probed with measurements that encompass carbonyl groups out past $6 \mu \mathrm{m}$, which could be taken into account with future development of the Trident concept.

The Triton Hopper concept study explored the ability of its design to generate propellant from the primarily nitrogen-ice surface of the moon, but also considered a wide instrument package to enable scientific characterization of Triton's surface and atmosphere (Landis et al., 2019; Oleson \& Landis, 2018). These instruments include a quadrupole mass spectrometer and a gas chromatograph (based on SAM, Sample Analysis at Mars) a V/UV/NIR spectrometer, a meteorological package, and an X-ray spectrometer. In context of increased oxidation of sedimented haze materials on the surface suggested by our results, the ability of the Hopper concept to use surface materials as propellant may be impacted. Recent efforts to use Pluto-like tholin to match the New Horizons surface observations of dark reddish material suggest highly porous structures of surface ice mixed with aerosols (Fayolle et al., 2021). If similar porous ice-aerosol structure is present on Triton, or if aerosol is entrained with the surface ice or snow, the oxidized refractory organic materials that make up the haze could prevent efficient intake of $\mathrm{N}_{2}$ to melt for propellant.

Neptune Odyssey, as a Flagship class concept, would be equipped with an extensive suite of instrumentation to enable its wide-ranging science goals, à la a Cassini for the Neptune system (Rymer et al., 2020). In terms of Triton science, Odyssey would investigate whether Triton is an ocean world, the source of its plumes, and broad scale compositional and dynamical processes of the atmosphere, surface, and interior. Relevant to our laboratory study here, this mission would carry imaging spectrometers in the UV and VIS-NIR, an ion and neutral mass spectrometer, a thermal plasma spectrometer, and an energetic charged particle detector. Such a mission would enable not only significant comparison to the Saturn-Titan system but also a far deeper understanding of Triton and Neptune than we currently possess. Nevertheless, the concept study also only studied a VIS-NIR spectrometer with range up to $5 \mu \mathrm{m}$ and an ion and neutral mass spectrometer with range up to $100 \mathrm{amu}$ (Rymer et al., 2020). Here we have shown that significantly large and complex molecules with significant oxygen incorporation can be produced under Triton-like atmosphere conditions, which could be best explored with spectroscopy beyond $5 \mu \mathrm{m}$ and mass spectrometry up to and beyond 450 amu. Lessons learned about the complex atmospheric chemistry of Titan and its unveiling (and the new questions uncovered) with Cassini should inform future mission development to explore and understand the correspondingly complex chemistry of Triton's atmosphere and surface.

\section{Conclusion}

We simulated haze formation in Triton's atmosphere using the PHAZER chamber and apparatus with a starting gas mixture of $0.5 \% \mathrm{CO}$ and $0.2 \% \mathrm{CH}_{4}$ in $\mathrm{N}_{2}$ at $90 \mathrm{~K}$. We then measured the production rate, composition, and spectra of the haze analogs produced with combustion analysis, very high resolution mass spectrometry, and transmittance and reflectance spectroscopy. We find that:

1. Oxygen is incorporated into the elemental composition of the solid tholin particles at approximately $10 \%$ by mass despite its inclusion in the form of $\mathrm{CO}$ in the original gas mixture at just $0.5 \%$.

2. When taking our Triton results and comparing them to previous PHAZER $\mathrm{N}_{2}-\mathrm{CH}_{4}-\mathrm{CO}$ experiments, the increase of $\mathrm{CO}$ over that of $\mathrm{CH}_{4}$ in the original gas mixture shifts the elemental composition away from carbon and toward a more nitrogen-rich structure, though more $\mathrm{CH}_{4}$ may generate larger absolute amounts of solid.

3. From very high resolution mass spectrometry measurements, we detect, as in previous studies, molecular formulas consistent with all 5 biological nucleotide bases, one non-biological nucleotide base, and several amino 
acid derivatives. Additionally, we observe the formula for glyceraldehyde, the simplest monosaccharide, for the first time from an $\mathrm{N}_{2}-\mathrm{CH}_{4}-\mathrm{CO}$ atmospheric experiment.

4. Transmission and reflectance spectra of the Triton tholin produce features attributable to O-H, N-H, C-H, $\mathrm{C} \equiv \mathrm{N}$, and $\mathrm{C} \equiv \mathrm{C}$ bonding. Most of these are also seen in spectra of similar tholin experimental data, but we also observe deeper and broader features, potentially attributable to oxygen bonds.

5. To take full advantage of the chemistry we observe in our laboratory setting, future Triton missions should carry instrumentation capable of probing high molecular weight compounds, such as mass spectrometers with high mass range ( $\geq 450 \mathrm{amu}$ ), and carbon-oxygen bonds, such as NIR spectrometers with spectral range out to at least $6.5 \mu \mathrm{m}$.

6. The exact $\mathrm{CO} / \mathrm{CH}_{4}$ mixing ratio in $\mathrm{N}_{2}$ atmospheres can dramatically affect the resulting haze chemistry and production rate. Since both Triton and Pluto undergo substantial atmospheric changes through sublimation of surface ices, their haze chemistry may also experience seasonal dependence.

Given our results along with the existing body of literature for Titan and Pluto, additional study is clearly motivated into the exact chemical pathways for haze formation between these three similar, yet distinct planetary bodies. The nature of this haze chemistry can affect the prebiotic inventories of these worlds, their climates and radiative balance, and seasonal cycling between their atmospheres and surfaces. As three worlds with $\mathrm{N}_{2}-\mathrm{CH}_{4}-$ $\mathrm{CO}$ atmospheres under different energetic regimes, Triton, Titan, and Pluto are themselves a fruitful laboratory for understanding carbon monoxide's dramatic influence on atmospheric chemistry.

\section{Appendix A: Complex Refractive Indices of Khare et al. (1994)}

Table A1

\begin{tabular}{|c|c|c|}
\hline \multirow[b]{2}{*}{ Wavelength (microns) } & \multicolumn{2}{|c|}{ Complex index of refraction } \\
\hline & Real index $(n)$ & Imaginary index $(k)$ \\
\hline 0.04959 & 0.86617 & 0.48009 \\
\hline 0.04999 & 0.87036 & 0.48617 \\
\hline 0.0504 & 0.87473 & 0.49233 \\
\hline 0.05081 & 0.87931 & 0.49861 \\
\hline 0.05123 & 0.88408 & 0.50499 \\
\hline 0.05166 & 0.88905 & 0.51148 \\
\hline 0.05209 & 0.89423 & 0.51807 \\
\hline 0.05253 & 0.89961 & 0.52479 \\
\hline 0.05298 & 0.9052 & 0.53161 \\
\hline 0.05344 & 0.91101 & 0.53856 \\
\hline 0.0539 & 0.91703 & 0.54561 \\
\hline 0.05438 & 0.92329 & 0.55279 \\
\hline 0.05486 & 0.92979 & 0.5601 \\
\hline 0.05535 & 0.93654 & 0.56755 \\
\hline 0.05585 & 0.94355 & 0.57513 \\
\hline 0.05635 & 0.95084 & 0.58285 \\
\hline 0.05687 & 0.95844 & 0.5907 \\
\hline 0.0574 & 0.96638 & 0.5987 \\
\hline 0.05793 & 0.97474 & 0.60686 \\
\hline 0.05848 & 0.9838 & 0.61515 \\
\hline 0.0588 & 0.98937 & 0.62 \\
\hline
\end{tabular}


Table A1

Continued

\begin{tabular}{|c|c|c|}
\hline \multirow[b]{2}{*}{ Wavelength (microns) } & \multicolumn{2}{|c|}{ Complex index of refraction } \\
\hline & Real index $(n)$ & Imaginary index $(k)$ \\
\hline 0.05904 & 0.99352 & 0.6236 \\
\hline 0.05961 & 1.0031 & 0.63202 \\
\hline 0.06018 & 1.0124 & 0.64033 \\
\hline 0.06078 & 1.0216 & 0.64856 \\
\hline 0.06138 & 1.0309 & 0.65665 \\
\hline 0.06199 & 1.0404 & 0.66463 \\
\hline 0.06262 & 1.05 & 0.67247 \\
\hline 0.06325 & 1.0598 & 0.68016 \\
\hline 0.06391 & 1.0697 & 0.68767 \\
\hline 0.06457 & 1.0799 & 0.69501 \\
\hline 0.06525 & 1.0904 & 0.70215 \\
\hline 0.06595 & 1.1011 & 0.70907 \\
\hline 0.06666 & 1.1122 & 0.71576 \\
\hline 0.06738 & 1.1235 & 0.72218 \\
\hline 0.06812 & 1.1352 & 0.72834 \\
\hline 0.06888 & 1.1473 & 0.73419 \\
\hline 0.06965 & 1.1598 & 0.73972 \\
\hline 0.07044 & 1.1727 & 0.74489 \\
\hline 0.07125 & 1.1862 & 0.74968 \\
\hline 0.07208 & 1.2002 & 0.75405 \\
\hline 0.07293 & 1.2149 & 0.75798 \\
\hline 0.0738 & 1.2303 & 0.76143 \\
\hline 0.07469 & 1.2466 & 0.76436 \\
\hline 0.0756 & 1.2639 & 0.76673 \\
\hline 0.07653 & 1.2825 & 0.76849 \\
\hline 0.07749 & 1.303 & 0.76959 \\
\hline 0.07847 & 1.3275 & 0.76999 \\
\hline 0.0785 & 1.3284 & 0.76999 \\
\hline 0.07947 & 1.3551 & 0.76999 \\
\hline 0.08 & 1.3693 & 0.76998 \\
\hline 0.08051 & 1.3826 & 0.76886 \\
\hline 0.08157 & 1.4069 & 0.76294 \\
\hline 0.08265 & 1.4277 & 0.75498 \\
\hline 0.08377 & 1.447 & 0.74602 \\
\hline 0.08492 & 1.4651 & 0.73642 \\
\hline 0.0861 & 1.4825 & 0.72633 \\
\hline 0.08731 & 1.4994 & 0.7158 \\
\hline 0.08856 & 1.516 & 0.70487 \\
\hline 0.08984 & 1.5324 & 0.69356 \\
\hline 0.09116 & 1.5492 & 0.68186 \\
\hline 0.0925 & 1.5678 & 0.66996 \\
\hline 0.09252 & 1.5681 & 0.66975 \\
\hline 0.09392 & 1.5864 & 0.65592 \\
\hline
\end{tabular}


Table A1

Continued

\begin{tabular}{|c|c|c|}
\hline \multirow[b]{2}{*}{ Wavelength (microns) } & \multicolumn{2}{|c|}{ Complex index of refraction } \\
\hline & Real index $(n)$ & Imaginary index $(k)$ \\
\hline 0.09537 & 1.6018 & 0.64162 \\
\hline 0.09686 & 1.6159 & 0.62687 \\
\hline 0.0984 & 1.6293 & 0.61166 \\
\hline 0.09998 & 1.6422 & 0.59597 \\
\hline 0.1016 & 1.6554 & 0.58 \\
\hline 0.10162 & 1.6556 & 0.5798 \\
\hline 0.10332 & 1.6683 & 0.56301 \\
\hline 0.10507 & 1.6791 & 0.54572 \\
\hline 0.10688 & 1.689 & 0.52785 \\
\hline 0.10875 & 1.6992 & 0.5094 \\
\hline 0.1097 & 1.7042 & 0.49999 \\
\hline 0.1107 & 1.7092 & 0.48935 \\
\hline 0.11271 & 1.7164 & 0.46748 \\
\hline 0.1148 & 1.721 & 0.44355 \\
\hline 0.1159 & 1.7214 & 0.42999 \\
\hline 0.11696 & 1.7214 & 0.41559 \\
\hline 0.1181 & 1.7184 & 0.40008 \\
\hline 0.11921 & 1.7143 & 0.38848 \\
\hline 0.1215 & 1.6999 & 0.37 \\
\hline 0.12155 & 1.6996 & 0.3698 \\
\hline 0.12398 & 1.6837 & 0.36075 \\
\hline 0.126 & 1.6713 & 0.35602 \\
\hline 0.12651 & 1.6685 & 0.35704 \\
\hline 0.12915 & 1.6585 & 0.36875 \\
\hline 0.1305 & 1.6598 & 0.3752 \\
\hline 0.13189 & 1.6615 & 0.38313 \\
\hline 0.13476 & 1.6846 & 0.40345 \\
\hline 0.1366 & 1.7114 & 0.4143 \\
\hline 0.13776 & 1.7275 & 0.4148 \\
\hline 0.14089 & 1.7588 & 0.37951 \\
\hline 0.14416 & 1.7658 & 0.31228 \\
\hline 0.1476 & 1.7544 & 0.25052 \\
\hline 0.1503 & 1.7315 & 0.24102 \\
\hline 0.1512 & 1.7264 & 0.248 \\
\hline 0.1532 & 1.7249 & 0.26535 \\
\hline 0.15498 & 1.7235 & 0.25909 \\
\hline 0.1559 & 1.7218 & 0.25492 \\
\hline 0.1585 & 1.7139 & 0.2095 \\
\hline 0.15895 & 1.7124 & 0.20955 \\
\hline 0.16313 & 1.6781 & 0.21051 \\
\hline 0.16754 & 1.6612 & 0.21258 \\
\hline 0.17219 & 1.6442 & 0.21626 \\
\hline 0.175 & 1.6347 & 0.2194 \\
\hline
\end{tabular}


Table A1

Continued

\begin{tabular}{|c|c|c|}
\hline \multirow[b]{2}{*}{ Wavelength (microns) } & \multicolumn{2}{|c|}{ Complex index of refraction } \\
\hline & Real index $(n)$ & Imaginary index $(k)$ \\
\hline 0.17711 & 1.6275 & 0.22896 \\
\hline 0.18 & 1.6158 & 0.24359 \\
\hline 0.18232 & 1.6067 & 0.24648 \\
\hline 0.1845 & 1.6083 & 0.24932 \\
\hline 0.18785 & 1.6121 & 0.30502 \\
\hline 0.1879 & 1.6123 & 0.30535 \\
\hline 0.19372 & 1.6575 & 0.33576 \\
\hline 0.195 & 1.667 & 0.33758 \\
\hline 0.19997 & 1.7024 & 0.32769 \\
\hline 0.2028 & 1.7168 & 0.3217 \\
\hline 0.2049 & 1.7271 & 0.31721 \\
\hline 0.20663 & 1.7355 & 0.31433 \\
\hline 0.2097 & 1.7503 & 0.3094 \\
\hline 0.21376 & 1.7694 & 0.3018 \\
\hline 0.215 & 1.7751 & 0.2992 \\
\hline 0.22139 & 1.8033 & 0.2748 \\
\hline 0.2264 & 1.8195 & 0.251 \\
\hline 0.22959 & 1.827 & 0.2328 \\
\hline 0.2364 & 1.8329 & 0.19104 \\
\hline 0.23842 & 1.8336 & 0.18305 \\
\hline 0.2471 & 1.8295 & 0.1491 \\
\hline 0.24796 & 1.8289 & 0.14573 \\
\hline 0.25829 & 1.8142 & 0.1051 \\
\hline 0.2587 & 1.8135 & 0.1035 \\
\hline 0.261 & 1.809 & 0.094679 \\
\hline 0.26952 & 1.791 & 0.082132 \\
\hline 0.273 & 1.7853 & 0.078 \\
\hline 0.28177 & 1.7732 & 0.069208 \\
\hline 0.29519 & 1.76 & 0.057937 \\
\hline 0.3 & 1.756 & 0.05411 \\
\hline 0.30995 & 1.7482 & 0.046466 \\
\hline 0.324 & 1.7376 & 0.0369 \\
\hline 0.32626 & 1.736 & 0.03559 \\
\hline 0.34439 & 1.7239 & 0.026779 \\
\hline 0.36 & 1.7143 & 0.0209 \\
\hline 0.36465 & 1.7117 & 0.019356 \\
\hline 0.37 & 1.7088 & 0.017767 \\
\hline 0.38 & 1.7036 & 0.01573 \\
\hline 0.38744 & 1.7001 & 0.015037 \\
\hline 0.39 & 1.699 & 0.014736 \\
\hline 0.4 & 1.6949 & 0.012734 \\
\hline 0.41327 & 1.6901 & 0.010889 \\
\hline 0.42 & 1.6878 & 0.010059 \\
\hline
\end{tabular}


Table A1

Continued

\begin{tabular}{|c|c|c|}
\hline \multirow[b]{2}{*}{ Wavelength (microns) } & \multicolumn{2}{|c|}{ Complex index of refraction } \\
\hline & Real index $(n)$ & Imaginary index $(k)$ \\
\hline 0.44 & 1.6817 & 0.0078597 \\
\hline 0.44279 & 1.6809 & 0.0075893 \\
\hline 0.46 & 1.6762 & 0.0061141 \\
\hline 0.47685 & 1.6721 & 0.0049604 \\
\hline 0.48 & 1.6714 & 0.004791 \\
\hline 0.5 & 1.6672 & 0.0041001 \\
\hline 0.51658 & 1.6641 & 0.0037892 \\
\hline 0.53 & 1.6619 & 0.003574 \\
\hline 0.55 & 1.6591 & 0.00328 \\
\hline 0.56355 & 1.6574 & 0.0031236 \\
\hline 0.57 & 1.6566 & 0.0030584 \\
\hline 0.59 & 1.6545 & 0.0028893 \\
\hline 0.6 & 1.6536 & 0.0028187 \\
\hline 0.61 & 1.6527 & 0.0027664 \\
\hline 0.6199 & 1.6518 & 0.0027253 \\
\hline 0.62 & 1.6518 & 0.0027249 \\
\hline 0.63 & 1.651 & 0.0026867 \\
\hline 0.64 & 1.6502 & 0.0026499 \\
\hline 0.65 & 1.6495 & 0.0026138 \\
\hline 0.66 & 1.6488 & 0.0025789 \\
\hline 0.67 & 1.6481 & 0.0025461 \\
\hline 0.68 & 1.6475 & 0.0025154 \\
\hline 0.68878 & 1.647 & 0.0024901 \\
\hline 0.69 & 1.6469 & 0.0024867 \\
\hline 0.7 & 1.6464 & 0.00246 \\
\hline 0.72 & 1.6454 & 0.0024134 \\
\hline 0.74 & 1.6445 & 0.0023746 \\
\hline 0.75 & 1.6441 & 0.0023575 \\
\hline 0.77 & 1.6433 & 0.0023314 \\
\hline 0.77488 & 1.6431 & 0.0023267 \\
\hline 0.79 & 1.6426 & 0.0023144 \\
\hline 0.8 & 1.6422 & 0.0022959 \\
\hline 0.82 & 1.6416 & 0.002197 \\
\hline 0.84 & 1.6411 & 0.0020675 \\
\hline 0.86 & 1.6405 & 0.0020144 \\
\hline 0.88 & 1.64 & 0.0019352 \\
\hline 0.88557 & 1.6399 & 0.0019106 \\
\hline 0.9 & 1.6396 & 0.0018452 \\
\hline 0.95 & 1.6385 & 0.0016617 \\
\hline 1 & 1.6376 & 0.0015323 \\
\hline 1.0332 & 1.637 & 0.0014503 \\
\hline 1.1 & 1.6361 & 0.001298 \\
\hline 1.2 & 1.635 & 0.0011223 \\
\hline
\end{tabular}


Table A1

Continued

\begin{tabular}{|c|c|c|}
\hline \multirow[b]{2}{*}{ Wavelength (microns) } & \multicolumn{2}{|c|}{ Complex index of refraction } \\
\hline & Real index $(n)$ & Imaginary index $(k)$ \\
\hline 1.2398 & 1.6346 & 0.0010529 \\
\hline 1.3 & 1.6341 & 0.00094895 \\
\hline 1.4 & 1.6333 & 0.0008075 \\
\hline 1.5 & 1.6326 & 0.00067144 \\
\hline 1.5498 & 1.6323 & 0.00062089 \\
\hline 1.6 & 1.632 & 0.0005775 \\
\hline 1.7 & 1.6314 & 0.00050698 \\
\hline 1.8 & 1.6307 & 0.0004711 \\
\hline 1.9 & 1.6302 & 0.00040131 \\
\hline 2 & 1.6297 & 0.00028705 \\
\hline 2.0663 & 1.6299 & 0.00026788 \\
\hline 2.1 & 1.6304 & 0.00025236 \\
\hline 2.1014 & 1.6304 & 0.00025099 \\
\hline 2.1376 & 1.6299 & 0.00019661 \\
\hline 2.1751 & 1.6296 & 0.00012606 \\
\hline 2.2 & 1.6294 & $9.36 \mathrm{E}-05$ \\
\hline 2.2139 & 1.6293 & $9.23 \mathrm{E}-05$ \\
\hline 2.2542 & 1.6291 & 9.49E-05 \\
\hline 2.2959 & 1.6288 & $9.58 \mathrm{E}-05$ \\
\hline 2.3 & 1.6288 & $9.58 \mathrm{E}-05$ \\
\hline 2.3392 & 1.6286 & $9.54 \mathrm{E}-05$ \\
\hline 2.3842 & 1.6283 & $9.44 \mathrm{E}-05$ \\
\hline 2.4 & 1.6282 & $9.18 \mathrm{E}-05$ \\
\hline 2.431 & 1.628 & $6.72 \mathrm{E}-05$ \\
\hline 2.4796 & 1.6276 & $1.37 \mathrm{E}-05$ \\
\hline 2.6379 & 1.6252 & $3.80 \mathrm{E}-06$ \\
\hline 2.6455 & 1.625 & $1.48 \mathrm{E}-05$ \\
\hline 2.6667 & 1.6242 & 0.00018093 \\
\hline 2.6952 & 1.6231 & 0.00041332 \\
\hline 2.7027 & 1.6228 & 0.00048627 \\
\hline 2.7397 & 1.6211 & 0.0018205 \\
\hline 2.7551 & 1.6205 & 0.0031149 \\
\hline 2.7778 & 1.6202 & 0.0051794 \\
\hline 2.8169 & 1.6207 & 0.0088768 \\
\hline 2.8177 & 1.6207 & 0.0089462 \\
\hline 2.8571 & 1.6232 & 0.011993 \\
\hline 2.8833 & 1.6253 & 0.013656 \\
\hline 2.8986 & 1.6267 & 0.01434 \\
\hline 2.9412 & 1.6309 & 0.013891 \\
\hline 2.9519 & 1.6318 & 0.013396 \\
\hline 2.9851 & 1.6338 & 0.011394 \\
\hline 3.0239 & 1.6353 & 0.0086455 \\
\hline 3.0303 & 1.6354 & 0.0082556 \\
\hline
\end{tabular}


Table A1

Continued

\begin{tabular}{|c|c|c|}
\hline \multirow[b]{2}{*}{ Wavelength (microns) } & \multicolumn{2}{|c|}{ Complex index of refraction } \\
\hline & Real index $(n)$ & Imaginary index $(k)$ \\
\hline 3.0769 & 1.6357 & 0.0064441 \\
\hline 3.0995 & 1.6357 & 0.0056691 \\
\hline 3.125 & 1.6356 & 0.0047953 \\
\hline 3.1746 & 1.6352 & 0.003073 \\
\hline 3.179 & 1.6352 & 0.0029589 \\
\hline 3.2258 & 1.6345 & 0.0020468 \\
\hline 3.2626 & 1.634 & 0.0014496 \\
\hline 3.2787 & 1.6338 & 0.0012418 \\
\hline 3.3333 & 1.6331 & 0.00084332 \\
\hline 3.3508 & 1.6329 & 0.00073703 \\
\hline 3.3898 & 1.6325 & 0.00053206 \\
\hline 3.4439 & 1.632 & 0.00030838 \\
\hline 3.4483 & 1.632 & 0.00029497 \\
\hline 3.5088 & 1.6315 & 0.00018484 \\
\hline 3.5423 & 1.6312 & 0.00015032 \\
\hline 3.5714 & 1.631 & 0.00012355 \\
\hline 3.6364 & 1.6307 & $6.89 \mathrm{E}-05$ \\
\hline 3.6465 & 1.6306 & $6.23 \mathrm{E}-05$ \\
\hline 3.7037 & 1.6303 & $3.46 \mathrm{E}-05$ \\
\hline 3.757 & 1.6301 & $3.06 \mathrm{E}-05$ \\
\hline 3.7736 & 1.63 & $2.83 \mathrm{E}-05$ \\
\hline 3.8461 & 1.6297 & 7.01E-06 \\
\hline 3.8744 & 1.6296 & $3.15 \mathrm{E}-06$ \\
\hline 4.065 & 1.6288 & $9.24 \mathrm{E}-06$ \\
\hline 4.0816 & 1.6287 & $1.48 \mathrm{E}-05$ \\
\hline 4.1169 & 1.6286 & $2.28 \mathrm{E}-05$ \\
\hline 4.1327 & 1.6285 & $3.79 \mathrm{E}-05$ \\
\hline 4.1459 & 1.6285 & $5.20 \mathrm{E}-05$ \\
\hline 4.1667 & 1.6285 & 7.53E-05 \\
\hline 4.1806 & 1.6285 & 0.00010292 \\
\hline 4.2087 & 1.6284 & 0.00057176 \\
\hline 4.2319 & 1.6284 & 0.00093355 \\
\hline 4.2553 & 1.6284 & 0.00063071 \\
\hline 4.268 & 1.6284 & 0.00075411 \\
\hline 4.2752 & 1.6284 & 0.00072837 \\
\hline 4.2827 & 1.6284 & 0.00069512 \\
\hline 4.2937 & 1.6284 & 0.00063988 \\
\hline 4.3122 & 1.6284 & 0.00047647 \\
\hline 4.329 & 1.6284 & 0.00035105 \\
\hline 4.3478 & 1.6284 & 0.00025447 \\
\hline 4.3649 & 1.6283 & 0.00020347 \\
\hline 4.3764 & 1.6283 & 0.00020734 \\
\hline 4.4053 & 1.6283 & 0.00024646 \\
\hline
\end{tabular}


Table A1

Continued

\begin{tabular}{|c|c|c|}
\hline \multirow[b]{2}{*}{ Wavelength (microns) } & \multicolumn{2}{|c|}{ Complex index of refraction } \\
\hline & Real index $(n)$ & Imaginary index $(k)$ \\
\hline 4.4189 & 1.6283 & 0.00026707 \\
\hline 4.4279 & 1.6283 & 0.00026953 \\
\hline 4.4287 & 1.6283 & 0.00027031 \\
\hline 4.4385 & 1.6283 & 0.00028135 \\
\hline 4.4444 & 1.6283 & 0.00028594 \\
\hline 4.4683 & 1.6282 & 0.0003004 \\
\hline 4.4763 & 1.6282 & 0.00031924 \\
\hline 4.5126 & 1.6281 & 0.00038237 \\
\hline 4.529 & 1.628 & 0.00039904 \\
\hline 4.5455 & 1.628 & 0.00041788 \\
\hline 4.562 & 1.628 & 0.00041695 \\
\hline 4.5788 & 1.6279 & 0.00042681 \\
\hline 4.5914 & 1.6279 & 0.00042622 \\
\hline 4.5919 & 1.6279 & 0.00042621 \\
\hline 4.6104 & 1.6279 & 0.00043211 \\
\hline 4.6211 & 1.6278 & 0.00042559 \\
\hline 4.6318 & 1.6278 & 0.0004171 \\
\hline 4.6512 & 1.6278 & 0.00040957 \\
\hline 4.6729 & 1.6278 & 0.00039339 \\
\hline 4.6904 & 1.6277 & 0.00039101 \\
\hline 4.7148 & 1.6277 & 0.00038963 \\
\hline 4.7259 & 1.6277 & 0.00038724 \\
\hline 4.7619 & 1.6276 & 0.00037006 \\
\hline 4.7685 & 1.6276 & 0.00036763 \\
\hline 4.7916 & 1.6276 & 0.00036111 \\
\hline 4.8263 & 1.6275 & 0.00035686 \\
\hline 4.8426 & 1.6275 & 0.00035122 \\
\hline 4.878 & 1.6274 & 0.000331 \\
\hline 4.8876 & 1.6274 & 0.0003271 \\
\hline 4.9116 & 1.6273 & 0.00032356 \\
\hline 4.931 & 1.6273 & 0.00030838 \\
\hline 4.953 & 1.6272 & 0.00030357 \\
\hline 4.9579 & 1.6272 & 0.00029346 \\
\hline 4.9592 & 1.6272 & 0.00029069 \\
\hline 4.9751 & 1.6272 & 0.00028162 \\
\hline 4.9826 & 1.6271 & 0.00027436 \\
\hline 4.985 & 1.6271 & 0.00027147 \\
\hline 5.0176 & 1.6271 & 0.0002642 \\
\hline 5.0302 & 1.627 & 0.0002509 \\
\hline 5.0454 & 1.627 & 0.00023347 \\
\hline 5.0607 & 1.627 & 0.00022653 \\
\hline 5.0839 & 1.6269 & 0.00021348 \\
\hline 5.1099 & 1.6269 & 0.00018989 \\
\hline
\end{tabular}


Table A1

Continued

\begin{tabular}{|c|c|c|}
\hline \multirow[b]{2}{*}{ Wavelength (microns) } & \multicolumn{2}{|c|}{ Complex index of refraction } \\
\hline & Real index $(n)$ & Imaginary index $(k)$ \\
\hline 5.1282 & 1.6268 & 0.00018452 \\
\hline 5.1414 & 1.6268 & 0.00017904 \\
\hline 5.152 & 1.6267 & 0.00016075 \\
\hline 5.1658 & 1.6267 & 0.00015504 \\
\hline 5.1733 & 1.6267 & 0.00015271 \\
\hline 5.2111 & 1.6266 & 0.00013814 \\
\hline 5.2356 & 1.6265 & 0.00010829 \\
\hline 5.2632 & 1.6264 & 4.57E-06 \\
\hline 5.4764 & 1.6252 & 9.97E-06 \\
\hline 5.5157 & 1.6248 & 4.84E-05 \\
\hline 5.5371 & 1.6247 & $9.39 \mathrm{E}-05$ \\
\hline 5.5556 & 1.6245 & 0.00010655 \\
\hline 5.6117 & 1.6241 & 0.00014623 \\
\hline 5.6338 & 1.6239 & 0.0001589 \\
\hline 5.6355 & 1.6239 & 0.00016004 \\
\hline 5.6818 & 1.6236 & 0.00023133 \\
\hline 5.7143 & 1.6234 & 0.00040635 \\
\hline 5.7504 & 1.6231 & 0.00077836 \\
\hline 5.7703 & 1.623 & 0.0010454 \\
\hline 5.7937 & 1.6228 & 0.001056 \\
\hline 5.8173 & 1.6227 & 0.0011696 \\
\hline 5.8275 & 1.6226 & 0.0012261 \\
\hline 5.8411 & 1.6225 & 0.0013033 \\
\hline 5.8823 & 1.6223 & 0.0022336 \\
\hline 5.9038 & 1.6223 & 0.0027969 \\
\hline 5.9277 & 1.6225 & 0.0035315 \\
\hline 5.9595 & 1.6228 & 0.0049669 \\
\hline 5.9844 & 1.6231 & 0.0065636 \\
\hline 5.9988 & 1.6233 & 0.007372 \\
\hline 6.0387 & 1.6239 & 0.0083311 \\
\hline 6.0606 & 1.6242 & 0.0090692 \\
\hline 6.0939 & 1.6246 & 0.010174 \\
\hline 6.1237 & 1.625 & 0.010441 \\
\hline 6.1652 & 1.6254 & 0.0093897 \\
\hline 6.199 & 1.6258 & 0.0074053 \\
\hline 6.2112 & 1.6259 & 0.0067416 \\
\hline 6.25 & 1.6263 & 0.0050224 \\
\hline 6.3211 & 1.627 & 0.003202 \\
\hline 6.3452 & 1.6272 & 0.0029247 \\
\hline 6.3816 & 1.6275 & 0.0025698 \\
\hline 6.4103 & 1.6277 & 0.0025929 \\
\hline 6.4267 & 1.6278 & 0.0024455 \\
\hline 6.4516 & 1.628 & 0.002453 \\
\hline
\end{tabular}


Table A1

Continued

\begin{tabular}{|c|c|c|}
\hline \multirow[b]{2}{*}{ Wavelength (microns) } & \multicolumn{2}{|c|}{ Complex index of refraction } \\
\hline & Real index $(n)$ & Imaginary index $(k)$ \\
\hline 6.4641 & 1.6281 & 0.0024269 \\
\hline 6.4809 & 1.6281 & 0.0024937 \\
\hline 6.5062 & 1.6283 & 0.0022592 \\
\hline 6.5253 & 1.6283 & 0.0021635 \\
\hline 6.536 & 1.6283 & 0.0021386 \\
\hline 6.566 & 1.6282 & 0.0021588 \\
\hline 6.5789 & 1.6282 & 0.0021253 \\
\hline 6.605 & 1.628 & 0.0021887 \\
\hline 6.6269 & 1.6279 & 0.0022664 \\
\hline 6.6445 & 1.6278 & 0.0022082 \\
\hline 6.6667 & 1.6276 & 0.0022567 \\
\hline 6.6845 & 1.6275 & 0.0022392 \\
\hline 6.7069 & 1.6273 & 0.0021785 \\
\hline 6.7249 & 1.6272 & 0.0020343 \\
\hline 6.7568 & 1.627 & 0.0019761 \\
\hline 6.7797 & 1.6269 & 0.0019367 \\
\hline 6.8027 & 1.6268 & 0.0018156 \\
\hline 6.8259 & 1.6267 & 0.0017637 \\
\hline 6.8353 & 1.6266 & 0.0017266 \\
\hline 6.8493 & 1.6266 & 0.0016424 \\
\hline 6.8776 & 1.6264 & 0.0015658 \\
\hline 6.8878 & 1.6264 & 0.0015594 \\
\hline 6.8966 & 1.6264 & 0.0015542 \\
\hline 6.9348 & 1.6263 & 0.0014976 \\
\hline 6.9541 & 1.6262 & 0.0015125 \\
\hline 6.9735 & 1.6262 & 0.0014798 \\
\hline 7.0126 & 1.6261 & 0.0014863 \\
\hline 7.0423 & 1.626 & 0.0014961 \\
\hline 7.0622 & 1.626 & 0.00145 \\
\hline 7.0972 & 1.6259 & 0.0014613 \\
\hline 7.1429 & 1.6258 & 0.0013825 \\
\hline 7.2411 & 1.6256 & 0.0012282 \\
\hline 7.2929 & 1.6255 & 0.0011018 \\
\hline 7.3314 & 1.6254 & 0.0010048 \\
\hline 7.3855 & 1.6253 & 0.00086976 \\
\hline 7.4074 & 1.6252 & 0.00086151 \\
\hline 7.4516 & 1.6251 & 0.00081194 \\
\hline 7.4738 & 1.625 & 0.00077195 \\
\hline 7.5244 & 1.6249 & 0.00069306 \\
\hline 7.57 & 1.6247 & 0.00062427 \\
\hline 7.593 & 1.6247 & 0.00055246 \\
\hline 7.6923 & 1.6244 & 0.0004644 \\
\hline 7.7399 & 1.6242 & 0.00044747 \\
\hline
\end{tabular}


Table A1

Continued

\begin{tabular}{|c|c|c|}
\hline \multirow[b]{2}{*}{ Wavelength (microns) } & \multicolumn{2}{|c|}{ Complex index of refraction } \\
\hline & Real index $(n)$ & Imaginary index $(k)$ \\
\hline 7.7488 & 1.6242 & 0.0004461 \\
\hline 7.8309 & 1.6239 & 0.00044524 \\
\hline 7.9681 & 1.6234 & 0.00042526 \\
\hline 8.1235 & 1.6229 & 0.00040333 \\
\hline 8.2169 & 1.6226 & 0.0003946 \\
\hline 8.2653 & 1.6224 & 0.00039497 \\
\hline 8.2988 & 1.6223 & 0.00039861 \\
\hline 8.3333 & 1.6222 & 0.00040807 \\
\hline 8.4104 & 1.622 & 0.00046919 \\
\hline 8.4818 & 1.6219 & 0.00068084 \\
\hline 8.5543 & 1.6217 & 0.0008507 \\
\hline 8.6059 & 1.6216 & 0.00095633 \\
\hline 8.6957 & 1.6214 & 0.0011349 \\
\hline 8.7489 & 1.6213 & 0.0011455 \\
\hline 8.8028 & 1.6211 & 0.00099961 \\
\hline 8.8557 & 1.621 & 0.00089411 \\
\hline 8.8731 & 1.621 & 0.00089171 \\
\hline 8.8968 & 1.6209 & 0.00097744 \\
\hline 8.9365 & 1.6208 & 0.0010451 \\
\hline 8.9928 & 1.6207 & 0.00086646 \\
\hline 9.0498 & 1.6205 & 0.00064804 \\
\hline 9.0909 & 1.6204 & 0.00054689 \\
\hline 9.1575 & 1.6203 & 0.00042919 \\
\hline 9.311 & 1.6199 & 0.00028792 \\
\hline 9.5369 & 1.6193 & 0.00025544 \\
\hline 9.5694 & 1.6192 & 0.00025166 \\
\hline 9.8912 & 1.6179 & 0.00022374 \\
\hline 10 & 1.6175 & 0.00018944 \\
\hline 10.204 & 1.6166 & $5.81 \mathrm{E}-05$ \\
\hline 10.267 & 1.6163 & $2.22 \mathrm{E}-06$ \\
\hline 10.73 & 1.6141 & 4.92E-06 \\
\hline 10.788 & 1.6138 & 0.00010207 \\
\hline 10.881 & 1.6133 & 0.00016606 \\
\hline 11.025 & 1.6126 & 0.00022157 \\
\hline 11.111 & 1.6122 & 0.0002574 \\
\hline 11.198 & 1.6118 & 0.00036696 \\
\hline 11.271 & 1.6114 & 0.00052602 \\
\hline 11.274 & 1.6114 & 0.00053403 \\
\hline 11.338 & 1.6111 & 0.00072421 \\
\hline 11.415 & 1.6107 & 0.00097889 \\
\hline 11.481 & 1.6103 & 0.00085287 \\
\hline 11.601 & 1.6097 & 0.00099804 \\
\hline 11.765 & 1.6089 & 0.0012961 \\
\hline
\end{tabular}


Table A1

Continued

\begin{tabular}{|c|c|c|}
\hline \multirow[b]{2}{*}{ Wavelength (microns) } & \multicolumn{2}{|c|}{ Complex index of refraction } \\
\hline & Real index $(n)$ & Imaginary index $(k)$ \\
\hline 12.034 & 1.6075 & 0.0019181 \\
\hline 12.315 & 1.6061 & 0.0027455 \\
\hline 12.398 & 1.6057 & 0.0029207 \\
\hline 12.407 & 1.6057 & 0.0029374 \\
\hline 12.5 & 1.6052 & 0.0030883 \\
\hline 12.739 & 1.6042 & 0.0038279 \\
\hline 12.937 & 1.6033 & 0.0044139 \\
\hline 13.004 & 1.603 & 0.0045061 \\
\hline 13.038 & 1.6029 & 0.0045918 \\
\hline 13.051 & 1.6028 & 0.0046262 \\
\hline 13.333 & 1.6013 & 0.0054012 \\
\hline 13.459 & 1.6006 & 0.0058752 \\
\hline 13.624 & 1.5997 & 0.0063335 \\
\hline 13.717 & 1.5992 & 0.0069037 \\
\hline 13.776 & 1.5989 & 0.0071453 \\
\hline 14.045 & 1.5976 & 0.008045 \\
\hline 14.286 & 1.5965 & 0.0090467 \\
\hline 14.586 & 1.5953 & 0.010598 \\
\hline 14.859 & 1.5943 & 0.012258 \\
\hline 14.993 & 1.5938 & 0.013134 \\
\hline 15.06 & 1.5936 & 0.01295 \\
\hline 15.385 & 1.5925 & 0.014476 \\
\hline 15.498 & 1.5922 & 0.014993 \\
\hline 16.051 & 1.5908 & 0.017311 \\
\hline 16.26 & 1.5902 & 0.018032 \\
\hline 16.531 & 1.5895 & 0.019364 \\
\hline 16.667 & 1.5891 & 0.019994 \\
\hline 17.123 & 1.5879 & 0.021403 \\
\hline 17.391 & 1.5872 & 0.022192 \\
\hline 17.637 & 1.5866 & 0.022923 \\
\hline 17.711 & 1.5864 & 0.022985 \\
\hline 17.825 & 1.5859 & 0.023049 \\
\hline 18.018 & 1.585 & 0.023671 \\
\hline 18.182 & 1.5842 & 0.023889 \\
\hline 18.416 & 1.5828 & 0.024375 \\
\hline 18.484 & 1.5824 & 0.024511 \\
\hline 18.553 & 1.582 & 0.024524 \\
\hline 18.657 & 1.5814 & 0.024404 \\
\hline 18.832 & 1.5802 & 0.024658 \\
\hline 19.011 & 1.579 & 0.024725 \\
\hline 19.074 & 1.5786 & 0.024867 \\
\hline 19.231 & 1.5775 & 0.025309 \\
\hline 19.531 & 1.575 & 0.024931 \\
\hline
\end{tabular}


Table A1

Continued

\begin{tabular}{lcc}
\hline & \multicolumn{2}{c}{ Complex index of refraction } \\
\cline { 2 - 3 } Wavelength (microns) & Real index $(n)$ & Imaginary index $(k)$ \\
\hline 19.685 & 1.5737 & 0.02467 \\
20 & 1.5708 & 0.024901 \\
20.663 & 1.5654 & 0.033283 \\
22.222 & 1.5615 & 0.057638 \\
22.542 & 1.5614 & 0.060825 \\
24.796 & 1.567 & 0.073943 \\
25 & 1.5662 & 0.07471 \\
27.551 & 1.5511 & 0.074067 \\
28.571 & 1.5456 & 0.076355 \\
30.995 & 1.5342 & 0.12651 \\
33.333 & 1.5457 & 0.17464 \\
35.423 & 1.5629 & 0.20358 \\
40 & 1.6181 & 0.24374 \\
41.327 & 1.6329 & 0.25248 \\
49.592 & 1.703 & 0.30692 \\
61.99 & 1.758 & 0.38857 \\
82.653 & 1.8148 & 0.52467 \\
123.98 & 1.8878 & 0.79687 \\
\hline
\end{tabular}

\section{Appendix B: Blank Mass Spectra}

Figures B1 and B2

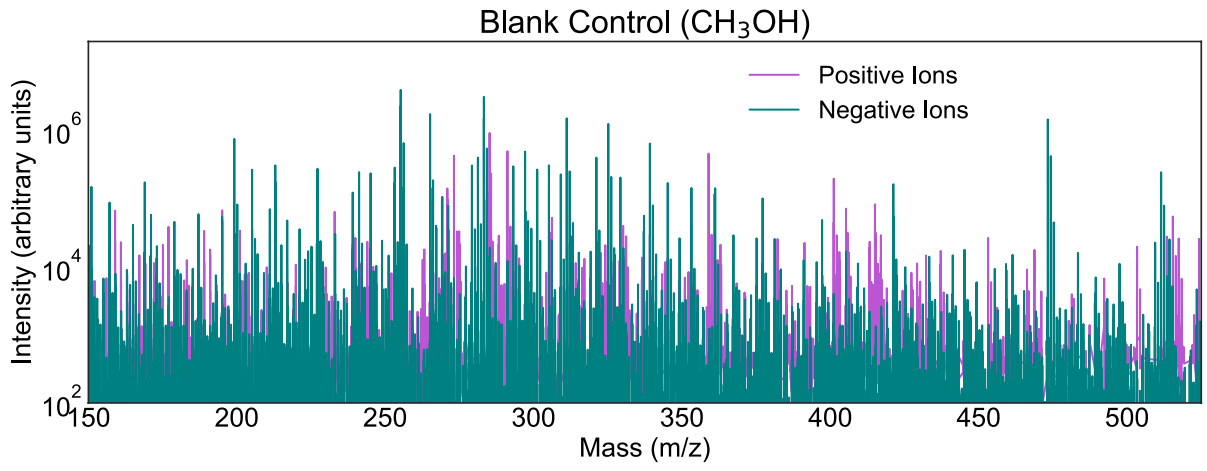

Figure B1. Positive (magenta) and negative (teal) ion mode mass spectra of the blank solution of $\mathrm{CH}_{3} \mathrm{OH}$ from $\mathrm{m} / \mathrm{z} 150$ to 525. The blank clearly lacks the structure of the actual sample (see Figure 4). Peaks greater than $2 \times 10^{5}$ present in the blank were removed from the Triton samples before peak assignments were made. These removed peaks constitute less than $0.03 \%$ of all peaks. 

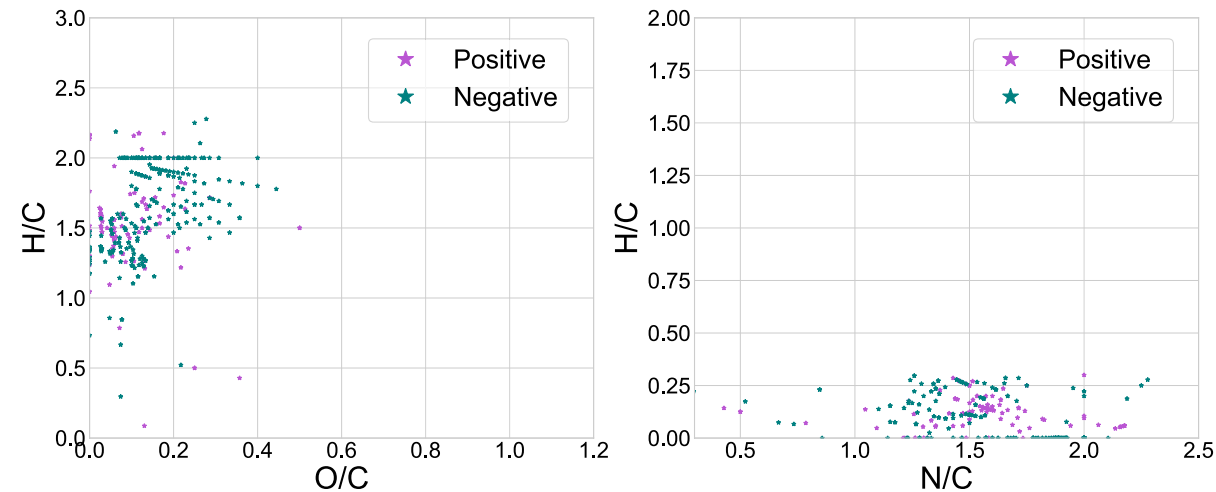

Figure B2. Positive (magenta) and negative (teal) ion mode van Krevelen diagrams of the blank solvent control. Left: H/C versus O/C. Right: H/C versus N/C.

\section{Appendix C: Fringe Correction}

Figure C1

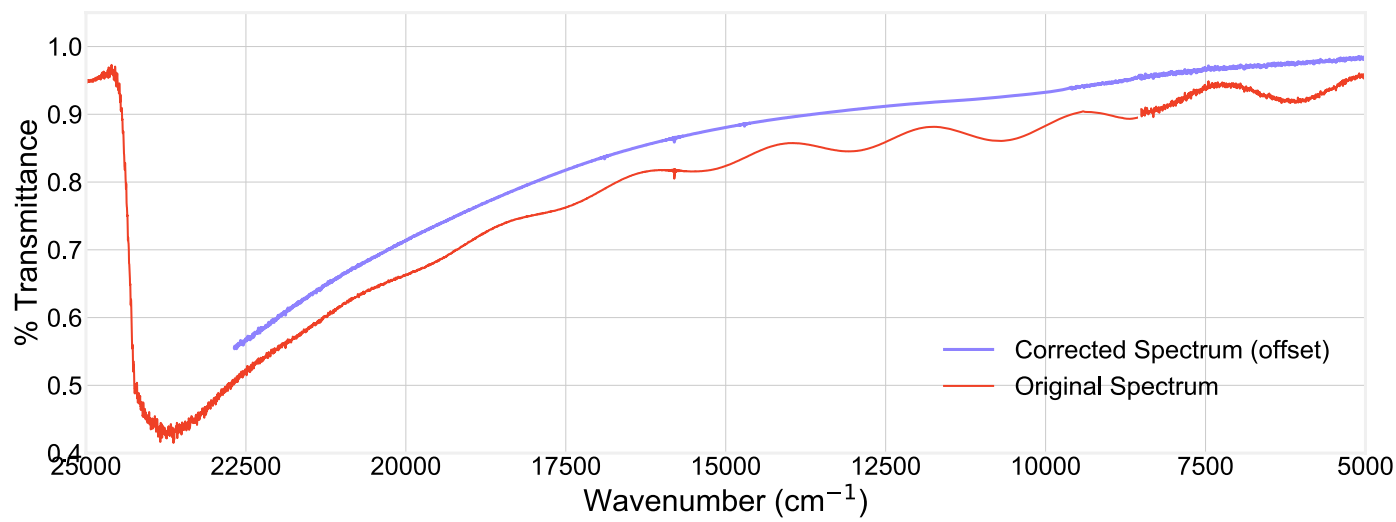

Figure C1. Raw (red) and fringe-corrected transmittance spectrum of the Triton haze analogs.

Acknowledgments

S. E. Moran was supported by NASA Earth and Space Science Fellowship Grant 80NSSC18K1109. S. E. Moran thanks R. M. Moran for enlightening discussions during development of the manuscript. This work is supported by the French National Research Agency in the framework of the Investissements d'Avenir program (ANR-15-IDEX-02), through the funding of the "Origin of Life" project of the Université Grenoble-Alpes and the French Space Agency (CNES) under their Exobiology and Solar System programs. C. Wolters acknowledges a PhD fellowship from CNES/ANR (ANR16-CE29-0015 2016-2021). The authors also thank Darrell Strobel for sharing the Khare Triton tholin data and Lora Jovanović for sharing the Pluto PAMPRE production rates.

\section{Data Availability Statement}

Data generated as a result of this analysis can be found in the Johns Hopkins University Data Archive (Moran et al., 2021): https://doi.org/10.7281/T1/BXJT0B.

\section{References}

Agnor, C. B., \& Hamilton, D. P. (2006). Neptune's capture of its moon Triton in a binary-planet gravitational encounter. Nature, 441(7090), 192-194. https://doi.org/10.1038/nature04792

Alcouffe, G., Cavarroc, M., Cernogora, G., Ouni, F., Jolly, A., Boufendi, L., \& Szopa, C. (2009). Capacitively coupled plasma used to simulate Titan's atmospheric chemistry. Plasma Sources Science and Technology, 19(1), 015008. https://doi.org/10.1088/0963-0252/19/1/015008

Bauer, J. M., Buratti, B. J., Li, J.-Y., Mosher, J. A., Hicks, M. D., Schmidt, B. E., \& Goguen, J. D. (2010). Direct detection of seasonal changes on Triton with Hubble Space Telescope. The Astrophysical Journal, 723(1), L49-L52. https://doi.org/10.1088/2041-8205/723/1/L49

Bernard, J. M., Coll, P., Coustenis, A., \& Raulin, F. (2003). Experimental simulation of Titan's atmosphere: Detection of ammonia and ethylene oxide. Planetary and Space Science, 51(14-15), 1003-1011. https://doi.org/10.1016/j.pss.2003.05.009

Berry, J. L., Ugelow, M. S., Tolbert, M. A., \& Browne, E. C. (2019). The influence of gas-phase chemistry on organic haze formation. The Astrophysical Journal Letters, 885(1), L6. https://doi.org/10.3847/2041-8213/ab4b5b

Bertrand, T., \& Forget, F. (2017). 3D modeling of organic haze in Pluto's atmosphere. Icarus, 287, 72-86. https://doi.org/10.1016/j. icarus.2017.01.016

Bonnet, J.-Y., Thissen, R., Frisari, M., Vuitton, V., Quirico, É., Orthous-Daunay, F.-R., et al. (2013). Compositional and structural investigation of HCN polymer through high resolution mass spectrometry. International Journal of Mass Spectrometry, 354, 193-203. https://doi. org/10.1016/j.ijms.2013.06.015 
Brassé, C., Muñoz, O., Coll, P., \& Raulin, F. (2015). Optical constants of Titan aerosols and their tholins analogs: Experimental results and modeling/observational data. Planetary and Space Science, 109, 159-174. https://doi.org/10.1016/j.pss.2015.02.012

Buratti, B. J., Bauer, J. M., Hicks, M. D., Hillier, J. K., Verbiscer, A., Hammel, H., et al. (2011). Photometry of Triton 1992-2004: Surface volatile transport and discovery of a remarkable opposition surge. Icarus, 212(2), 835-846. https://doi.org/10.1016/j.icarus.2011.01.012

Cable, M. L., Hörst, S. M., Hodyss, R., Beauchamp, P., Smith, M. A., \& Willis, P. (2012). Titan tholins: Simulating Titan organic chemistry in the Cassini-Huygens era. Chemical Reviews, 3, 1882-1909. https://doi.org/10.1021/cr200221x

Cheng, A. F., Summers, M. E., Gladstone, G. R., Strobel, D. F., Young, L. A., Lavvas, P. P., et al. (2017). Haze in Pluto's atmosphere. Icarus, 290, 112-133. https://doi.org/10.1016/j.icarus.2017.02.024

Christophe, B., Spilker, L. J., Anderson, J. D., André, N., Asmar, S. W., Aurnou, J., et al. (2012). OSS (Outer Solar System): A fundamental and planetary physics mission to Neptune, Triton and the Kuiper Belt. Experimental Astronomy, 34(2), 203-242. https://doi.org/10.1007/ s10686-012-9309-y

Coll, P., Bernard, J.-M., Navarro-González, R., \& Raulin, F. (2003). Oxirane: An exotic oxygenated organic compound on Titan? TheAstrophysical Journal, 598(1), 700-703. https://doi.org/10.1086/378792

Conrath, B., Flasar, F. M., Hanel, R., Kunde, V., Maguire, W., Pearl, J., et al. (1989). Infrared observations of the Neptunian system. Science, 246(4936), 1454-1459. https://doi.org/10.1126/science.246.4936.1454

Cruikshank, D. P., Materese, C. K., Pendleton, Y. J., Boston, P. J., Grundy, W. M., Schmitt, B., et al. (2019). Prebiotic chemistry of Pluto. Astrobiology, 19(7), 831-848. https://doi.org/10.1089/ast.2018.1927

Cruikshank, D. P., Roush, T. L., Owen, T. C., Geballe, T. R., de Bergh, C., Schmitt, B., et al. (1993). Ices on the surface of Triton. Science, 261(5122), 742-745. https://doi.org/10.1126/science.261.5122.742

Cruikshank, D. P., Schmitt, B., Roush, T. L., Owen, T. C., Quirico, E., Geballe, T. R., et al. (2000). Water ice on Triton. Icarus, 147(1), 309-316. https://doi.org/10.1006/icar.2000.6451

DeWitt, H. L., Trainer, M. G., Pavlov, A. A., Hasenkopf, C. A., Aiken, A. C., Jimenez, J. L., et al. (2009). Reduction in haze formation rate on prebiotic Earth in the presence of hydrogen. Astrobiology, 9(5), 447-453. https://doi.org/10.1089/ast.2008.0289

Elliot, J. L., Hammel, H. B., Wasserman, L. H., Franz, O. G., McDonald, S. W., Person, M. J., et al. (1998). Global warming on Triton. Nature, 393(6687), 765-767. https://doi.org/10.1038/31651

Fayolle, M., Quirico, E., Schmitt, B., Jovanovic, L., Gautier, T., Carrasco, N., et al. (2021). Testing tholins as analogues of the dark reddish material covering Pluto's Cthulhu region. Icarus, 367, 114574. https://doi.org/10.1016/j.icarus.2021.114574

Fletcher, L. N., Helled, R., Roussos, E., Jones, G., Charnoz, S., André, N., et al. (2020). Ice giant systems: The scientific potential of orbital missions to Uranus and Neptune. Planetary and Space Science, 191, 105030. https://doi.org/10.1016/j.pss.2020.105030

Gao, P., Fan, S., Wong, M. L., Liang, M.-C., Shia, R.-L., Kammer, J. A., et al. (2017). Constraints on the microphysics of Pluto's photochemical haze from New Horizons observations. Icarus, 287, 116-123. https://doi.org/10.1016/j.icarus.2016.09.030

Gautier, T., Carrasco, N., Schmitz-Afonso, I., Touboul, D., Szopa, C., Buch, A., \& Pernot, P. (2014). Nitrogen incorporation in Titan's tholins inferred by high resolution Orbitrap mass spectrometry and gas chromatography-mass spectrometry. Earth and Planetary Science Letters, 404 , 33-42. https://doi.org/10.1016/j.eps1.2014.07.011

Gautier, T., Schmitz-Afonso, I., Touboul, D., Szopa, C., Buch, A., \& Carrasco, N. (2016). Development of HPLC-Orbitrap method for identification of N-bearing molecules in complex organic material relevant to planetary environments. Icarus, 275, 259-266. https://doi.org/10.1016/j. icarus.2016.03.007

Gautier, T., Sebree, J. A., Li, X., Pinnick, V. T., Grubisic, A., Loeffler, M. J., et al. (2017). Influence of trace aromatics on the chemical growth mechanisms of Titan aerosol analogues. Planetary and Space Science, 140, 27-34. https://doi.org/10.1016/j.pss.2017.03.012

Gavilan, L., Carrasco, N., Vrønning Hoffmann, S., Jones, N. C., \& Mason, N. J. (2018). Organic aerosols in anoxic and oxic atmospheres of Earthlike Exoplanets: VUV-MIR spectroscopy of CHON tholins. The Astrophysical Journal, 861, 110. https://doi.org/10.3847/1538-4357/aac8df

Gladstone, G. R., Stern, S. A., Ennico, K., Olkin, C. B., Weaver, H. A., Young, L. A., et al. (2016). The atmosphere of Pluto as observed by New Horizons. Science, 351(6279), aad8866. https://doi.org/10.1126/science.aad8866

Gronoff, G., Lilensten, J., \& Modolo, R. (2009). Ionization processes in the atmosphere of Titan-II. Electron precipitation along magnetic field lines. Astronomy and Astrophysics, 506(2), 965-970. https://doi.org/10.1051/0004-6361/200912125

Gronoff, G., Mertens, C., Lilensten, J., Desorgher, L., Flückiger, E., \& Velinov, P. (2011). Ionization processes in the atmosphere of Titan. III. Ionization by high-Z nuclei cosmic rays. Astronomy and Astrophysics, 529, A143. https://doi.org/10.1051/0004-6361/201015675

Grundy, W. M., Bertrand, T., Binzel, R. P., Buie, M. W., Buratti, B. J., Cheng, A. F., et al. (2018). Pluto's haze as a surface material. Icarus, 314, 232-245. https://doi.org/10.1016/j.icarus.2018.05.019

Grundy, W. M., Buie, M. W., \& Spencer, J. R. (2002). Spectroscopy of Pluto and Triton at 3-4 microns: Possible evidence for wide distribution of nonvolatile solids. The Astronomical Journal, 124(4), 2273-2278. https://doi.org/10.1086/342933

Grundy, W. M., Young, L. A., Stansberry, J. A., Buie, M. W., Olkin, C. B., \& Young, E. F. (2010). Near-infrared spectral monitoring of Triton with IRTF/SpeX II: Spatial distribution and evolution of ices. Icarus, 205(2), 594-604. https://doi.org/10.1016/j.icarus.2009.08.005

Gudipati, M. S., Jacovi, R., Couturier-Tamburelli, I., Lignell, A., \& Allen, M. (2013). Photochemical activity of Titan's low-altitude condensed haze. Nature Communications, 4, 1648. https://doi.org/10.1038/ncomms2649

Hammond, N. P., Parmenteir, E. M., \& Barr, A. C. (2018). Compaction and melt transport in ammonia-rich ice shells: Implications for the evolution of Triton. Journal of Geophysical Research: Planets, 123(12), 3105-3118. https://doi.org/10.1029/2018JE005781

Hansen, C. J., McEwen, A. S., Ingersoll, A. P., \& Terrile, R. J. (1990). Surface and airborne evidence for plumes and winds on Triton. Science, 250(4979), 421-424. https://doi.org/10.1126/science.250.4979.421

Hansen, C. J., \& Paige, D. A. (1992). A thermal model for the seasonal nitrogen cycle on Triton. Icarus, 99(2), 273-288. https://doi. org/10.1016/0019-1035(92)90146-X

Hartle, R. E., Sittler, E. C., Neubauer, F. M., Johnson, R. E., Smith, H. T., Crary, F., et al. (2006). Initial interpretation of Titan plasma interaction as observed by the Cassini plasma spectrometer: Comparisons with Voyager 1. Planetary and Space Science, 54(12), 1211-1224. https://doi. org/10.1016/j.pss.2006.05.029

Hasenkopf, C. A., Beaver, M. R., Trainer, M. G., Langley Dewitt, H., Freedman, M. A., Toon, O. B., et al. (2010). Optical properties of Titan and early Earth haze laboratory analogs in the mid-visible. Icarus, 207(2), 903-913. https://doi.org/10.1016/j.icarus.2009.12.015

He, C., Hörst, S. M., Lewis, N. K., Moses, J. I., Kempton, E. M. R., Marley, M. S., et al. (2019). Gas phase chemistry of cool exoplanet atmospheres: Insight from laboratory simulations. ACS Earth and Space Chemistry, 3(1), 39-50. https://doi.org/10.1021/acsearthspacechem.8b00133

He, C., Hörst, S. M., Lewis, N. K., Yu, X., Moses, J. I., Kempton, E. M. R., et al. (2018). Laboratory simulations of haze formation in the atmospheres of super-Earths and mini-Neptunes: Particle color and size distribution. The Astrophysical Journal Letters, 856(1), L3. https://doi. org/10.3847/2041-8213/aab42b 
He, C., Hörst, S. M., Lewis, N. K., Yu, X., Moses, J. I., McGuiggan, P., et al. (2020). Haze formation in warm $\mathrm{H}_{2}$-rich exoplanet atmospheres. The Planetary Science Journal, 1(2), 51. https://doi.org/10.3847/PSJ/abb1a4

He, C., Hörst, S. M., Radke, M. J., \& Yant, M. (2022). Optical constants of Titan haze analogue from 0.4 to $3.5 \mu$ m: Determined using vacuum spectroscopy. The Planetary Science Journal. accepted.

He, C., Hörst, S. M., Riemer, S., Sebree, J. A., Pauley, N., \& Vuitton, V. (2017). Carbon monoxide affecting planetary atmospheric chemistry. The Astrophysical Journal, 841, L31. https://doi.org/10.3847/2041-8213/aa74cc

Herbert, F., \& Sandel, B. R. (1991). $\mathrm{CH}_{4}$ and haze in Triton's lower atmosphere. Journal of Geophysical Research, 96, 19241-19252. https://doi. org/10.1029/91JA01821

Hillier, J. K., Buratti, B. J., Hofgartner, J. D., Hicks, M. D., Devins, S., \& Kivrak, L. (2021). Characteristics of Pluto's haze and surface from an analytic radiative transfer model. The Planetary Science Journal, 2(1), 11. https://doi.org/10.3847/PSJ/abbdaf

Hillier, J. K., Helfenstein, P., Verbiscer, A., \& Veverka, J. (1991). Voyager photometry of Triton: Haze and surface photometric properties. Journal of Geophysical Research, 96, 19203-19209. https://doi.org/10.1029/91JA01736

Hodyss, R., Howard, H. R., Johnson, P. V., Goguen, J. D., \& Kanik, I. (2011). Formation of radical species in photolyzed $\mathrm{CH}_{4}: \mathrm{N}_{2}$ ices. Icarus, 214(2), 748-753. https://doi.org/10.1016/j.icarus.2011.05.023

Hofstadter, M., Simon, A., Atreya, S., Banfield, D., Fortney, J. J., Hayes, A., et al. (2019). Uranus and Neptune missions: A study in advance of the next Planetary Science Decadal Survey. Planetary and Space Science, 177, 104680. https://doi.org/10.1016/j.pss.2019.06.004

Holler, B. J., Young, L. A., Grundy, W. M., \& Olkin, C. B. (2016). On the surface composition of Triton's southern latitudes. Icarus, 267, 255-266. https://doi.org/10.1016/j.icarus.2015.12.027

Hörst, S. M. (2011). Post-Cassini investigations of Titan atmospheric chemistry (Unpublished doctoral dissertation. PhD Thesis). University of Arizona.

Hörst, S. M., He, C., Lewis, N. K., Kempton, E. M. R., Marley, M. S., Morley, C. V., et al. (2018). Haze production rates in super-Earth and mini-Neptune atmosphere experiments. Nature Astronomy, 2, 303-306. https://doi.org/10.1038/s41550-018-0397-0

Hörst, S. M., He, C., Ugelow, M. S., Jellinek, A. M., Pierrehumbert, R. T., \& Tolbert, M. A. (2018). Exploring the atmosphere of neoproterozoic Earth: The effect of $\mathrm{O}_{2}$ on haze formation and composition. The Astrophysical Journal, 858, 119. https://doi.org/10.3847/1538-4357/aabd7d

Hörst, S. M., \& Tolbert, M. A. (2013). In situ measurements of the size and density of Titan aerosol analogs. The Astrophysical Journal Letters, 770(1), L10. https://doi.org/10.1088/2041-8205/770/1/L10

Hörst, S. M., \& Tolbert, M. A. (2014). The effect of carbon monoxide on planetary haze formation. The Astrophysical Journal, $781(1)$, 53. https://doi.org/10.1088/0004-637X/781/1/53

Hörst, S. M., Yelle, R. V., Buch, A., Carrasco, N., Cernogora, G., Dutuit, O., et al. (2012). Formation of amino acids and nucleotide bases in a Titan atmosphere simulation experiment. Astrobiology, 12(9), 809. https://doi.org/10.1089/ast.2011.0623

Hörst, S. M., Yoon, Y. H., Ugelow, M. S., Parker, A. H., Li, R., de Gouw, J. A., \& Tolbert, M. A. (2018). Laboratory investigations of Titan haze formation: In situ measurement of gas and particle composition. Icarus, 301, 136-151. https://doi.org/10.1016/j.icarus.2017.09.039

Imanaka, H., Khare, B. N., Elsila, J. E., Bakes, E. L. O., McKay, C. P., Cruikshank, D. P., et al. (2004). Laboratory experiments of Titan tholin formed in cold plasma at various pressures: Implications for nitrogen-containing polycyclic aromatic compounds in Titan haze. Icarus, 168(2), 344-366. https://doi.org/10.1016/j.icarus.2003.12.014

Johnson, P. V., Hodyss, R., Chernow, V. F., Lipscomb, D. M., \& Goguen, J. D. (2012). Ultraviolet photolysis of amino acids on the surface of icy Solar System bodies. Icarus, 221(2), 800-805. https://doi.org/10.1016/j.icarus.2012.09.005

Jovanović, L., Gautier, T., Broch, L., Protopapa, S., Bertrand, T., Rannou, P., et al. (2021). Optical constants of Pluto aerosol analogues from UV to near-IR. Icarus, 362, 114398. https://doi.org/10.1016/j.icarus.2021.114398

Jovanović, L., Gautier, T., Vuitton, V., Wolters, C., Bourgalais, J., Buch, A., et al. (2020). Chemical composition of Pluto aerosol analogues. Icarus, 346, 113774. https://doi.org/10.1016/j.icarus.2020.113774

Khare, B. N., Sagan, C., Arakawa, E. T., Suits, F., Callcott, T. A., \& Williams, M. W. (1984). Optical constants of organic tholins produced in a simulated Titanian atmosphere: From soft X-ray to microwave frequencies. Icarus, 60(1), 127-137. https://doi.org/10.1016/0019-1035(84)90142-8

Khare, B. N., Sagan, C., Heinrich, M., Thompson, W. R., Arakawa, E. T., Tuminello, P. S., \& Clark, M. (1994). Optical constants of Triton Tholin: Preliminary results. In AAS/Division for Planetary Sciences Meeting Abstracts \#26 (Vol. 26, p. 30.03).

Kim, S., Kramer, R. W., \& Hatcher, P. G. (2003). Graphical method for analysis of ultrahigh-resolution broadband mass spectra of natural organic matter, the van Krevelen diagram. Analytical Chemistry, 75(20), 5336-5344. https://doi.org/10.1021/ac034415p

Krasnopolsky, V. A. (2012). Titan's photochemical model: Further update, oxygen species, and comparison with Triton and Pluto. Planetary and Space Science, 73(1), 318-326. https://doi.org/10.1016/j.pss.2012.08.013

Krasnopolsky, V. A. (2020). A photochemical model of Pluto's atmosphere and ionosphere. Icarus, 335, 113374. https://doi.org/10.1016/j. icarus.2019.07.008

Krasnopolsky, V. A., \& Cruikshank, D. P. (1995). Photochemistry of Triton's atmosphere and ionosphere. Journal of Geophysical Research, 100, 21271-21286. https://doi.org/10.1029/95je01904

Krasnopolsky, V. A., Sandel, B. R., \& Herbert, F. (1992). Properties of haze in the atmosphere of Triton. Journal of Geophysical Research, 97(E7), 11695-11700. https://doi.org/10.1029/92JE00945

Krasnopolsky, V. A., Sandel, B. R., Herbert, F., \& Vervack, R. J. (1993). Temperature, N 2 , and N density profiles of Triton's atmosphere: Observations and model. Journal of Geophysical Research, 98(E2), 3065-3078. https://doi.org/10.1029/92JE02680

Krimigis, S. M., Armstrong, T. P., Axford, W. I., Bostrom, C. O., Cheng, A. F., Gloeckler, G., et al. (1989). Hot plasma and energetic particles in Neptune's magnetosphere. Science, 246(4936), 1483-1489. https://doi.org/10.1126/science.246.4936.1483

Kutsop, N. W., Hayes, A. G., Buratti, B. J., Corlies, P. M., Ennico, K., Fan, S., et al. (2021). Pluto's haze abundance and size distribution from limb scatter observations by MVIC. The Planetary Science Journal, 2(3), 91. https://doi.org/10.3847/psj/abdcaf

Landis, G., Oleson, S., Abel, P., Bur, M. J. C., Colozza, A. J., Faller, B., et al. (2019). Missions to triton and pluto using a hopper vehicle with in-situ refueling. In 70th International Astronautical Congress.

Lavvas, P. P., Coustenis, A., \& Vardavas, I. M. (2008). Coupling photochemistry with haze formation in Titan's atmosphere, Part II: Results and validation with Cassini/Huygens data. Planetary and Space Science, 56(1), 67-99. https://doi.org/10.1016/j.pss.2007.05.027

Lavvas, P. P., Lellouch, E., Strobel, D. F., Gurwell, M. A., Cheng, A. F., Young, L. A., \& Gladstone, G. R. (2021). A major ice component in Pluto's haze. Nature Astronomy, 5, 289-297. https://doi.org/10.1038/s41550-020-01270-3

Lavvas, P. P., Yelle, R. V., Koskinen, T., Bazin, A., Vuitton, V., Vigren, E., et al. (2013). Aerosol growth in Titan's ionosphere. Proceedings of the National Academy of Science, 110(8), 2729-2734. https://doi.org/10.1073/pnas.1217059110

Lellouch, E., de Bergh, C., Sicardy, B., Ferron, S., \& Käufl, H. U. (2010). Detection of CO in Triton's atmosphere and the nature of surface-atmosphere interactions. Astronomy and Astrophysics, 512, L8. https://doi.org/10.1051/0004-6361/201014339 
Lewis, B., Stansberry, J., Holler, B., Grundy, W., Schmitt, B., Protopapa, S., et al. (2020). Distribution and energy balance of Pluto's nitrogen ice, as seen by New Horizons in 2015. Icarus, 356, 113633. https://doi.org/10.1016/j.icarus.2020.113633

Liang, M.-C., Yung, Y. L., \& Shemansky, D. E. (2007). Photolytically generated aerosols in the mesosphere and thermosphere of Titan. The Astrophysical Journal Letters, 661(2), L199-L202. https://doi.org/10.1086/518785

Luspay-Kuti, A., Mandt, K., Jessup, K.-L., Kammer, J., Hue, V., Hamel, M., \& Filwett, R. (2017). Photochemistry on Pluto-I. Hydrocarbons and aerosols. Monthly Notices of the Royal Astronomical Society, 472(1), 104-117. https://doi.org/10.1093/mnras/stx1362

Lyons, J. R., Yung, Y. L., \& Allen, M. (1992). Solar control of the upper atmosphere of Triton. Science, 256(5054), 204-206. https://doi. org/10.1126/science.11540928

Maillard, J., Carrasco, N., Schmitz-Afonso, I., Gautier, T., \& Afonso, C. (2018). Comparison of soluble and insoluble organic matter in analogues of Titan's aerosols. Earth and Planetary Science Letters, 495, 185-191. https://doi.org/10.1016/j.eps1.2018.05.014

Masters, A., Achilleos, N., Agnor, C. B., Campagnola, S., Charnoz, S., Christophe, B., et al. (2014). Neptune and Triton: Essential pieces of the Solar System puzzle. Planetary and Space Science, 104, 108-121. https://doi.org/10.1016/j.pss.2014.05.008

Materese, C. K., Cruikshank, D. P., Sandford, S. A., Imanaka, H., \& Nuevo, M. (2015). Ice chemistry on outer solar system bodies: Electron radiolysis of $\mathrm{N}_{2}-\mathrm{CH}_{4}$, and CO-containing ices. Acta Pathologica Japonica, 812(2), 150. https://doi.org/10.1088/0004-637X/812/2/150

Materese, C. K., Cruikshank, D. P., Sandford, S. A., Imanaka, H., Nuevo, M., \& White, D. W. (2014). Ice chemistry on outer solar system bodies: Carboxylic acids, nitriles, and urea detected in refractory residues produced from the UV photolysis of $\mathrm{N}_{2}: \mathrm{CH}_{4}: \mathrm{CO}$-containing ices. The Astrophysical Journal, 788(2), 111. https://doi.org/10.1088/0004-637X/788/2/111

McDonald, G. D., Thompson, W. R., Heinrich, M., Khare, B. N., \& Sagan, C. (1994). Chemical investigation of Titan and Triton tholins. Icarus, 108(1), 137-145. https://doi.org/10.1006/icar.1994.1046

McKinnon, W. B. (1984). On the origin of Triton and Pluto. Nature, 311(5984), 355-358. https://doi.org/10.1038/311355a0

Merlin, F., Lellouch, E., Quirico, E., \& Schmitt, B. (2018). Triton's surface ices: Distribution, temperature and mixing state from VLT/SINFONI observations. Icarus, 314, 274-293. https://doi.org/10.1016/j.icarus.2018.06.003

Moore, M. H., \& Hudson, R. L. (2003). Infrared study of ion-irradiated $\mathrm{N}_{2}$-dominated ices relevant to Triton and Pluto: Formation of HCN and HNC. Icarus, 161(2), 486-500. https://doi.org/10.1016/S0019-1035(02)00037-4

Moran, S. E., Hörst, S. M., He, C., Radke, M. J., Sebree, J. A., Izenberg, N. R., et al. (2021). Data associated with the publication: Triton haze analogues: The role of carbon monoxide in haze formation. Johns Hopkins University Data Archive, V1. https://doi.org/10.7281/T1/BXJT0B

Moran, S. E., Hörst, S. M., Vuitton, V., He, C., Lewis, N. K., Fland inet, L., et al. (2020). Chemistry of temperate super-Earth and mini-Neptune atmospheric hazes from laboratory experiments. The Planetary Science Journal, 1(1), 17. https://doi.org/10.3847/PSJ/ab8eae

Neish, C. D., Somogyi, Á., \& Smith, M. A. (2010). Titan's primordial soup: Formation of amino acids via low-temperature hydrolysis of tholins. Astrobiology, 10(3), 337-347. https://doi.org/10.1089/ast.2009.0402

Neri, F., Saitta, G., \& Chiofalo, S. (1987). A simple procedure to remove the interference fringes from optical spectra. Journal of Physics E: Scientific Instruments, 20(7), 894-896. https://doi.org/10.1088/0022-3735/20/7/015

Nimmo, F., \& Spencer, J. R. (2015). Powering Triton's recent geological activity by obliquity tides: Implications for Pluto geology. Icarus, 246, 2-10. https://doi.org/10.1016/j.icarus.2014.01.044

Ohno, K., Zhang, X., Tazaki, R., \& Okuzumi, S. (2021). Haze formation on Triton. The Astrophysical Journal, 912(1), 37. https://doi. org/10.3847/1538-4357/abee82

Oleson, S. R., \& Landis, G. (2018). Triton hopper: Exploring Neptune's captured Kuiper Belt Object. In V. Badescu \& K. Zacny (Eds.), Outer solar system (pp. 367-428). Springer. https://doi.org/10.1007/978-3-319-73845-1_9

Olkin, C. B., Elliot, J. L., Hammel, H. B., Cooray, A. R., McDonald, S. W., Foust, J. A., et al. (1997). The thermal structure of Triton's atmosphere: Results from the 1993 and 1995 occultations. Icarus, 129(1), 178-201. https://doi.org/10.1006/icar.1997.5757

Orthous-Daunay, F.-R., Thissen, R., \& Vuitton, V. (2020). Measured mass to stoichiometric formula through exhaustive search. In F. Salama \& H. Linnartz (Eds.), Laboratory astrophysics: From observations to interpretation (Vol. 350, pp. 193-199). https://doi.org/10.1017/ S1743921319008032

Pollack, J. B., Schwartz, J. M., \& Rages, K. (1990). Scatterers in Triton's atmosphere: Implications for the seasonal volatile cycle. Science, 250(4979), 440-443. https://doi.org/10.1126/science.250.4979.440

Porco, C. C., Baker, E., Barbara, J., Beurle, K., Brahic, A., Burns, J. A., et al. (2005). Imaging of Titan from the Cassini spacecraft. Nature, 434(7030), 159-168. https://doi.org/10.1038/nature03436

Prockter, L. M., Mitchell, K. L., Howett, C. J. A., Smythe, W. D., Sutin, B. M., Bearden, D. A., \& Frazier, W. E. (2019). Exploring Triton with Trident: A discovery class mission. In Lunar and Planetary Science Conference (p. 3188).

Protopapa, S., Olkin, C. B., Grundy, W. M., Li, J.-Y., Verbiscer, A., Cruikshank, D. P., et al. (2020). Disk-resolved photometric properties of Pluto and the coloring materials across its surface. The Astronomical Journal, 159(2), 74. https://doi.org/10.3847/1538-3881/ab5e82

Rages, K., \& Pollack, J. B. (1992). Voyager imaging of Triton's clouds and hazes. Icarus, 99(2), 289-301. https://doi. org/10.1016/0019-1035(92)90147-Y

Ruf, A., Le Sergeant d'Hendecourt, L., \& Schmitt-Kopplin, P. (2018). Data-driven astrochemistry: One step further within the origin of life puzzle. Life, 8 , 18. https://doi.org/10.3390/life 8020018

Rymer, A. M., Clyde, B., \& Runyon, K., \& the Neptune-Odyssey team. (2020). Neptune Odyssey: Mission to the Neptune-Triton system. LPI Contributions, 2547, 6031.

Schenk, P. M., \& Zahnle, K. (2007). On the negligible surface age of Triton. Icarus, 192(1), 135-149. https://doi.org/10.1016/j.icarus.2007.07.004

Sciamma-O'Brien, E., Carrasco, N., Szopa, C., Buch, A., \& Cernogora, G. (2010). Titan's atmosphere: An optimal gas mixture for aerosol production? Icarus, 209(2), 704-714. https://doi.org/10.1016/j.icarus.2010.04.009

Sebree, J. A., Roach, M. C., Shipley, E. R., He, C., \& Hörst, S. M. (2018). Detection of prebiotic molecules in plasma and photochemical aerosol analogs using GC/MS/MS techniques. The Astrophysical Journal, 865(2), 133. https://doi.org/10.3847/1538-4357/aadba1

Smith, B. A., Soderblom, L. A., Banfield, D., Barnet, C., Basilevksy, A. T., Beebe, R. F., et al. (1989). Voyager 2 at Neptune: Imaging science results. Science, 246(4936), 1422-1449. https://doi.org/10.1126/science.246.4936.1422

Stansberry, J. A., Spencer, J., \& Linscott, I. (2015). Voyager IRIS measurements of Triton's Thermal emission: Implications for Pluto? In AAS/ Division for Planetary Sciences Meeting Abstracts \#47 (pp. 210-232).

Stern, S. A., Bagenal, F., Ennico, K., Gladstone, G. R., Grundy, W. M., McKinnon, W. B., et al. (2015). The Pluto system: Initial results from its exploration by New Horizons. Science, 350(6258), aad1815. https://doi.org/10.1126/science.aad1815

Stern, S. A., \& McKinnon, W. B. (2000). Triton's surface age and impactor population revisited in light of Kuiper Belt fluxes: Evidence for small Kuiper Belt objects and recent geological activity. The Astronomical Journal, 119(2), 945-952. https://doi.org/10.1086/301207

Stern, S. A., Trafton, L. M., \& Flynn, B. (1995). Rotationally resolved studies of the mid-ultraviolet spectrum of Triton. II. HST surface and atmospheric results. The Astronomical Journal, 109, 2855. https://doi.org/10.1086/117491 
Stone, E. C., Cummings, A. C., Looper, M. D., Selesnick, R. S., Lal, N., McDonald, F. B., et al. (1989). Energetic charged particles in the magnetosphere of Neptune. Science, 246(4936), 1489-1494. https://doi.org/10.1126/science.246.4936.1489

Strobel, D. F., \& Summers, M. E. (1995). Triton's upper atmosphere and ionosphere. In Neptune and Triton (pp. 1107-1148).

Strobel, D. F., \& Zhu, X. (2017). Comparative planetary nitrogen atmospheres: Density and thermal structures of Pluto and Triton. Icarus, 291, 55-64. https://doi.org/10.1016/j.icarus.2017.03.013

Szopa, C., Cernogora, G., Boufendi, L., Correia, J. J., \& Coll, P. (2006). PAMPRE: A dusty plasma experiment for Titan's tholins production and study. Planetary and Space Science, 54(4), 394-404. https://doi.org/10.1016/j.pss.2005.12.012

Telfer, M. W., Parteli, E. J. R., Radebaugh, J., Beyer, R. A., Bertrand, T., Forget, F., et al. (2018). Dunes on Pluto. Science, $360(6392), 992-997$. https://doi.org/10.1126/science.aao2975

Thompson, W. R., Singh, S. K., Khare, B. N., \& Sagan, C. (1989). Triton: Stratospheric molecules and organic sediments. Geophyscal Research Letters, 16(8), 981-984. https://doi.org/10.1029/GL016i008p00981

Tomasko, M. G., Doose, L., Engel, S., Dafoe, L. E., West, R., Lemmon, M., et al. (2008). A model of Titan's aerosols based on measurements made inside the atmosphere. Planetary and Space Science, 56(5), 669-707. https://doi.org/10.1016/j.pss.2007.11.019

Trainer, M. G., Pavlov, A. A., Curtis, D. B., McKay, C. P., Worsnop, D. R., Delia, A. E., et al. (2004). Haze aerosols in the atmosphere of early Earth: Manna from heaven. Astrobiology, 4(4), 409-419. https://doi.org/10.1089/ast.2004.4.409

Trainer, M. G., Pavlov, A. A., Dewitt, H. L., Jimenez, J. L., McKay, C. P., Toon, O. B., \& Tolbert, M. A. (2006). Inaugural article: Organic haze on Titan and the early Earth. Proceedings of the National Academy of Science, 103(48), 18035-18042. https://doi.org/10.1073/pnas.0608561103

Tran, B. N., Force, M., Briggs, R. G., Ferris, J. P., Persans, P., \& Chera, J. J. (2008). Titan's atmospheric chemistry: Photolysis of gas mixtures containing hydrogen cyanide and carbon monoxide at 185 and $254 \mathrm{~nm}$. Icarus, 193(1), 224-232. https://doi.org/10.1016/j.icarus.2007.09.010

Tyler, G. L., Sweetnam, D. N., Anderson, J. D., Borutzki, S. E., Campbell, J. K., Eshleman, V. R., et al. (1989). Voyager radio science observations of Neptune and Triton. Science, 246(4936), 1466-1473. https://doi.org/10.1126/science.246.4936.1466

van Krevelen, D. W. (1950). Graphical-statistical method for the study of structure and reaction processes of coal. Fuel, 29, 269-284.

Vuitton, V., Bonnet, J.-Y., Frisari, M., Thissen, R., Quirico, E., Dutuit, O., et al. (2010). Very high resolution mass spectrometry of HCN polymers and tholins. Faraday Discussions, 147, 495. https://doi.org/10.1039/c003758c

Vuitton, V., Moran, S. E., He, C., Wolters, C., Flandinet, L., Orthous-Daunay, F.-R., et al. (2021). $\mathrm{H}_{2} \mathrm{SO}_{4}$ and organosulfur compounds in laboratory analogue aerosols of warm high-metallicity exoplanet atmospheres. The Planetary Science Journal, 2(1), 2. https://doi.org/10.3847/PSJ/abc558

Vuitton, V., Yelle, R. V., Klippenstein, S. J., Hörst, S. M., \& Lavvas, P. P. (2019). Simulating the density of organic species in the atmosphere of Titan with a coupled ion-neutral photochemical model. Icarus, 324, 120-197. https://doi.org/10.1016/j.icarus.2018.06.013

Wolters, C., Flandinet, L., He, C., Isa, J., Orthous-Daunay, F.-R., Thissen, R., et al. (2020). Enhancing data acquisition for the analysis of complex organic matter in direct-infusion Orbitrap mass spectrometry using micro-scans. Rapid Communications in Mass Spectrometry, 34(15), e8818. https://doi.org/10.1002/rcm. 8818

Wong, M. L., Fan, S., Gao, P., Liang, M.-C., Shia, R.-L., Yung, Y. L., et al. (2017). The photochemistry of Pluto's atmosphere as illuminated by New Horizons. Icarus, 287, 110-115. https://doi.org/10.1016/j.icarus.2016.09.028

Yelle, R. V., Lunine, J. I., Pollack, J. B., \& Brown, R. H. (1995). Lower atmospheric structure and surface-atmosphere interactions on Triton. In Neptune and Triton (pp. 1031-1105).

Young, L. A., \& Stern, S. A. (2001). Ultraviolet observations of Triton in 1999 with the Space Telescope Imaging Spectrograph: 2150-3180 A spectroscopy and disk-integrated photometry. The Astronomical Journal, 122(1), 449-456. https://doi.org/10.1086/322062

Yu, X., Hörst, S. M., He, C., McGuiggan, P., \& Bridges, N. T. (2017). Direct measurement of interparticle forces of Titan aerosol analogs ("tholin") using atomic force microscopy. Journal of Geophysical Research: Planets, 122(12), 2610-2622. https://doi.org/10.1002/2017JE005437

Zhang, X., Strobel, D. F., \& Imanaka, H. (2017). Haze heats Pluto's atmosphere yet explains its cold temperature. Nature, 551, 352-355. https:// doi.org/10.1038/nature24465 